九州大学学術情報リポジトリ

Kyushu University Institutional Repository

Asymptotic behavior of solutions to the compressible Navier-Stokes equation around a time-periodic parallel flow

Brezina, Jan

Graduate School of Mathematics, Kyushu University

http://hdl. hand le. net/2324/24652

出版情報 : MI Preprint Series. 2012-10，2012-08-23. 九州大学大学院数理学研究院 バージョン：

権利関係 : 


\section{Preprint Series}

Kyushu University

The Global COE Program

Math-for-Industry Education \& Research Hub

\section{Asymptotic behavior of solutions \\ to the compressible \\ Navier-Stokes equation around a time-periodic parallel flow}

\section{Jan Brezina}

MI 2012-10

( Received August 23, 2012 )

Faculty of Mathematics

Kyushu University

Fukuoka, JAPAN 


\title{
Asymptotic behavior of solutions to the compressible Navier-Stokes equation around a time-periodic parallel flow
}

\author{
Jan Březina *
}

\begin{abstract}
The global in time existence of strong solutions to the compressible Navier-Stokes equation around time-periodic parallel flows in $\mathbb{R}^{n}, n \geq 2$, is established under smallness conditions on Reynolds number, Mach number and initial perturbations. Furthermore, it is proved for $n=2$ that the asymptotic leading part of solutions is given by a solution of one-dimensional viscous Burgers equation multiplied by timeperiodic function. In the case $n \geq 3$ the asymptotic leading part of solutions is given by a solution of $n$-1-dimensional heat equation with convective term multiplied by time-periodic function.
\end{abstract}

\section{Mathematics Subject Classification}

Keywords. Compressible Navier-Stokes equation, global existence, asymptotic behavior, time-periodic, viscous Burgers equation.

\section{Introduction}

In this paper we study the stability of solutions around a time-periodic parallel flow to the compressible Navier-Stokes equation with time-periodic external force and time-periodic boundary conditions.

We consider the system of equations

$$
\begin{gathered}
\partial_{\tilde{t}} \widetilde{\rho}+\operatorname{div}(\widetilde{\rho v})=0, \\
\widetilde{\rho}\left(\partial_{\widetilde{t}} \widetilde{v}+\widetilde{v} \cdot \nabla \widetilde{v}\right)-\mu \Delta \widetilde{v}-\left(\mu+\mu^{\prime}\right) \nabla \operatorname{div} \widetilde{v}+\nabla \widetilde{P}(\widetilde{\rho})=\widetilde{\rho} \widetilde{g},
\end{gathered}
$$

in an $n$ dimensional infinite layer $\Omega_{\ell}=\mathbb{R}^{n-1} \times(0, \ell)$ :

$$
\begin{aligned}
\Omega_{\ell}=\{\widetilde{x} & ={ }^{T}\left(\widetilde{x}^{\prime}, \widetilde{x}_{n}\right) ; \\
\widetilde{x}^{\prime} & \left.={ }^{T}\left(\widetilde{x}_{1}, \ldots, \widetilde{x}_{n-1}\right) \in \mathbb{R}^{n-1}, 0<\widetilde{x}_{n}<\ell\right\} .
\end{aligned}
$$

Here, $n \geq 2 ; \widetilde{\rho}=\widetilde{\rho}(\widetilde{x}, \widetilde{t})$ and $\widetilde{v}={ }^{T}\left(\widetilde{v}^{1}(\widetilde{x}, \widetilde{t}), \ldots, \widetilde{v}^{n}(\widetilde{x}, \widetilde{t})\right)$ denote the unknown density and velocity at time $\widetilde{t} \geq 0$ and position $\widetilde{x} \in \Omega_{\ell}$, respectively; $\widetilde{P}$ is the pressure, smooth function of $\widetilde{\rho}$, where for given $\rho_{*}>0$ we assume

$$
\widetilde{P}^{\prime}\left(\rho_{*}\right)>0
$$

$\mu$ and $\mu^{\prime}$ are the viscosity coefficients that are assumed to be constants satisfying $\mu>0, \frac{2}{n} \mu+\mu^{\prime} \geq 0 ;$ div, $\nabla$ and $\Delta$ denote the usual divergence, gradient and Laplacian with respect to $\widetilde{x}$. Here and in what follows ${ }^{T}$. denotes the transposition.

In (1.2) $\widetilde{\boldsymbol{g}}$ is assumed to have the form

$$
\widetilde{\boldsymbol{g}}={ }^{T}\left(\widetilde{g}^{1}\left(\widetilde{x}_{n}, \widetilde{t}\right), 0, \ldots, 0, \widetilde{g}^{n}\left(\widetilde{x}_{n}\right)\right),
$$

with $\widetilde{g}^{1}$ being a $\tau$-periodic function in time, where $\tau>0$.

The system (1.1)-(1.2) is considered under boundary condition

$$
\left.\widetilde{v}\right|_{\widetilde{x}_{n}=0}=\widetilde{V}^{1}(t) \boldsymbol{e}_{1},\left.\quad \widetilde{v}\right|_{\widetilde{x}_{n}=\ell}=0,
$$

and initial condition

$$
\left.(\widetilde{\rho}, \widetilde{v})\right|_{\widetilde{t}=0}=\left(\widetilde{\rho}_{0}, \widetilde{v}_{0}\right)
$$

where $\widetilde{V}^{1}$ is a $\tau$-periodic function of time and $\boldsymbol{e}_{1}={ }^{T}(1,0, \ldots, 0) \in \mathbb{R}^{n}$.

Under suitable conditions on $\widetilde{\boldsymbol{g}}$ and $\widetilde{V}^{1}$, problem (1.1)-(1.3) has smooth time-periodic solution $\bar{u}_{p}=$ $T\left(\bar{\rho}_{p}, \bar{v}_{p}\right)$ satisfying

\footnotetext{
*Graduate School of Mathematics, Kyushu University, Fukuoka 819-0395, JAPAN
} 


$$
\begin{gathered}
\bar{\rho}_{p}=\bar{\rho}_{p}\left(\widetilde{x}_{n}\right) \geq \widetilde{\rho}_{1}, \quad \frac{1}{\ell} \int_{0}^{\ell} \bar{\rho}_{p}\left(\widetilde{x}_{n}\right) d \widetilde{x}_{n}=\rho_{*}, \\
\bar{v}_{p}={ }^{T}\left(\bar{v}_{p}^{1}\left(\widetilde{x}_{n}, \widetilde{t}\right), 0, \ldots, 0\right), \quad \bar{v}_{p}^{1}\left(\widetilde{x}_{n}, \widetilde{t}+\tau\right)=\bar{v}_{p}^{1}\left(\widetilde{x}_{n}, \widetilde{t}\right),
\end{gathered}
$$

for a positive constant $\widetilde{\rho}_{1}$.

The aim of this paper is to give an asymptotic description of large time behavior of perturbations from $\bar{u}_{p}$ when Reynolds and Mach numbers are sufficiently small.

To formulate the problem for perturbations, we introduce the following dimensionless variables:

$$
\widetilde{x}=\ell x, \quad \widetilde{t}=\frac{\ell}{V} t, \quad \widetilde{v}=V v, \quad \widetilde{\rho}=\rho_{*} \rho, \quad \widetilde{P}=\rho_{*} V^{2} P,
$$

with

$$
\widetilde{w}=V w, \quad \widetilde{\phi}=\rho_{*} \gamma^{-2} \phi, \quad \widetilde{V}^{1}=V V^{1}, \quad \widetilde{\boldsymbol{g}}=\frac{\mu V}{\rho_{*} \ell^{2}} \boldsymbol{g},
$$

where

$$
\gamma=\frac{\sqrt{\widetilde{P}^{\prime}\left(\rho_{*}\right)}}{V}, \quad V=\frac{\rho_{*} \ell^{2}}{\mu}\left\{\left|\partial_{\hat{t}} \widetilde{V}^{1}\right|_{C^{0}(\mathbb{R})}+\left|\widetilde{g}^{1}\right|_{C^{0}(\mathbb{R} \times[0, \ell])}\right\}+\left|\widetilde{V}^{1}\right|_{C^{0}(\mathbb{R})}>0 .
$$

In this paper we assume $V>0$. Under this change of variables the domain $\Omega_{\ell}$ is transformed into $\Omega=$ $\mathbb{R}^{n-1} \times(0,1)$; and $g^{1}\left(x_{n}, t\right), V^{1}(t)$ are periodic in $t$ with period $T>0$ defined by

$$
T=\frac{V}{\ell} \tau \text {. }
$$

The time-periodic solution $\bar{u}_{p}$ is transformed into $u_{p}={ }^{T}\left(\rho_{p}, v_{p}\right)$ satisfying

$$
\begin{gathered}
\rho_{p}=\rho_{p}\left(x_{n}\right)>0, \int_{0}^{1} \rho_{p}\left(x_{n}\right) d x_{n}=1, \\
v_{p}={ }^{T}\left(v_{p}^{1}\left(x_{n}, t\right), 0, \ldots, 0\right), v_{p}^{1}\left(x_{n}, t+T\right)=v_{p}^{1}\left(x_{n}, t\right) .
\end{gathered}
$$

It then follows that the perturbation $u(t)={ }^{T}(\phi(t), w(t)) \equiv{ }^{T}\left(\gamma^{2}\left(\rho(t)-\rho_{p}\right), v(t)-v_{p}(t)\right)$ is governed by the following system of equations

$$
\begin{gathered}
\partial_{t} \phi+v_{p}^{1} \partial_{x_{1}} \phi+\gamma^{2} \operatorname{div}\left(\rho_{p} w\right)=f^{0} \\
\partial_{t} w-\frac{\nu}{\rho_{p}} \Delta w-\frac{\widetilde{\nu}}{\rho_{p}} \nabla \operatorname{div} w+v_{p}^{1} \partial_{x_{1}} w+\left(\partial_{x_{n}} v_{p}^{1}\right) w^{n} \boldsymbol{e}_{1} \\
+\frac{\nu}{\gamma^{2} \rho_{p}^{2}}\left(\partial_{x_{n}}^{2} v_{p}^{1}\right) \phi \boldsymbol{e}_{1}+\nabla\left(\frac{P^{\prime}\left(\rho_{p}\right)}{\gamma^{2} \rho_{p}} \phi\right)=\boldsymbol{f}, \\
\left.w\right|_{\partial \Omega}=0 \\
\left.(\phi, w)\right|_{t=0}=\left(\phi_{0}, w_{0}\right),
\end{gathered}
$$

where $f^{0}$ and $\boldsymbol{f}={ }^{T}\left(f^{1}, \cdots, f^{n}\right)$ denote nonlinearities, i.e.,

$$
\begin{gathered}
f^{0}=-\operatorname{div}(\phi w), \\
\boldsymbol{f}=-w \cdot \nabla w+\frac{\nu \phi}{\gamma^{2} \rho_{p}^{2}}\left(-\Delta w+\frac{\partial_{x_{n}}^{2} v_{p}^{1}}{\rho_{p} \gamma^{2}} \phi \boldsymbol{e}_{1}\right)-\frac{\nu \phi^{2}}{\gamma^{2} \rho_{p}^{2}\left(\gamma^{2} \rho_{p}+\phi\right)}\left(-\Delta w+\frac{\partial_{x_{n}}^{2} v_{p}^{1}}{\rho_{p} \gamma^{2}} \phi \boldsymbol{e}_{1}\right) \\
-\frac{\widetilde{\nu} \phi}{\rho_{p}\left(\gamma^{2} \rho_{p}+\phi\right)} \nabla \operatorname{div} w+\frac{\phi}{\gamma^{2} \rho_{p}} \nabla\left(\frac{P^{\prime}\left(\rho_{p}\right)}{\gamma^{2} \rho_{p}} \phi\right)-\frac{1}{2 \gamma^{4} \rho_{p}} \nabla\left(P^{\prime \prime}\left(\rho_{p}\right) \phi^{2}\right)+\widetilde{P}_{3}\left(\rho_{p}, \phi, \partial_{x} \phi\right), \\
\widetilde{P}_{3}\left(\rho_{p}, \phi, \partial_{x} \phi\right)=\frac{\phi^{3}}{\gamma^{4}\left(\gamma^{2} \rho_{p}+\phi\right) \rho_{p}^{3}} \nabla P\left(\rho_{p}\right)+\frac{\phi \nabla\left(P^{\prime \prime}\left(\rho_{p}\right) \phi^{2}\right)}{2 \gamma^{4} \rho_{p}\left(\gamma^{2} \rho_{p}+\phi\right)} \\
-\frac{\phi^{2} \nabla\left(P^{\prime}\left(\rho_{p}\right) \phi\right)}{\gamma^{4} \rho_{p}^{2}\left(\gamma^{2} \rho_{p}+\phi\right)}-\frac{1}{2 \gamma^{4}\left(\gamma^{2} \rho_{p}+\phi\right)} \nabla\left(\phi^{3} P_{3}\left(\rho_{p}, \phi\right)\right),
\end{gathered}
$$

with

$$
P_{3}\left(\rho_{p}, \phi\right)=\int_{0}^{1}(1-\theta)^{2} P^{\prime \prime \prime}\left(\theta \gamma^{-2} \phi+\rho_{p}\right) d \theta
$$


Here, div,$\nabla$ and $\Delta$ denote the usual divergence, gradient and Laplacian with respect to $x ; \nu, \nu^{\prime}$ and $\widetilde{\nu}$ are the non-dimensional parameters:

$$
\nu=\frac{\mu}{\rho_{*} \ell V}, \quad \nu^{\prime}=\frac{\mu^{\prime}}{\rho_{*} \ell V}, \quad \widetilde{\nu}=\nu+\nu^{\prime} .
$$

We note that the Reynolds number $R e$ and Mach number $M a$ are given by $R e=\nu^{-1}$ and $M a=\gamma^{-1}$, respectively. See [1] for the derivation of (1.5)-(1.8).

In the case $g^{1}$ and $V^{1}$ do not depend on $t$, problem (1.1)-(1.3) has a stationary parallel flow. The stability of stationary parallel flows were studied in $[5,6,7,11]$. It was shown in [6] and [7] that the stationary parallel flow is asymptotically stable under sufficiently small initial perturbations in $H^{m}(\Omega) \cap L^{1}(\Omega)$ with $m \geq[n / 2]+1$, provided that $R e \ll 1, M a \ll 1$ and density of the parallel flow is sufficiently close to a positive constant. Furthermore, the asymptotic behavior of perturbations from the stationary parallel flow is described by $n-1$ dimensional linear heat equation in the case $n \geq 3([6])$ and by one-dimensional viscous Burgers equation in the case $n=2([7])$.

The case of time-periodic parallel flows was considered in $[1,2]$ for $R e \ll 1$ and $M a \ll 1$. In $[1,2]$ the authors investigated the linearized problem, i.e., (1.5)-(1.8) with $\left(f^{0}, \boldsymbol{f}\right)=(0,0)$, which is written as

$$
\partial_{t} u+L(t) u=0,\left.\quad w\right|_{x_{n}=0,1}=0,\left.\quad u\right|_{t=s}=u_{0} .
$$

Here, $u={ }^{T}(\phi, w)$ and $L(t)$ is operator of the form

$$
\begin{aligned}
L(t) & =\left(\begin{array}{cc}
v_{p}^{1}(t) \partial_{x_{1}} & \gamma^{2} \operatorname{div}\left(\rho_{p} \cdot\right) \\
\nabla\left(\frac{P^{\prime}\left(\rho_{p}\right)}{\gamma^{2} \rho_{p}} \cdot\right) & -\frac{\nu}{\rho_{p}} \Delta I_{n}-\frac{\widetilde{\nu}}{\rho_{p}} \nabla \operatorname{div}
\end{array}\right) \\
& +\left(\begin{array}{cc}
0 & 0 \\
\frac{\nu}{\gamma^{2} \rho_{p}^{2}} \partial_{x_{n}}^{2} v_{p}^{1}(t) \boldsymbol{e}_{1} & v_{p}^{1}(t) \partial_{x_{1}} I_{n}+\left(\partial_{x_{n}} v_{p}^{1}(t)\right) \boldsymbol{e}_{1}{ }^{T} \boldsymbol{e}_{n}
\end{array}\right) .
\end{aligned}
$$

Note that $L(t)$ satisfies $L(t)=L(t+T)$.

In $[1,2]$ spectral properties of the solution operator $U(t, s)$ for $(1.9)$ were studied by using Fourier transform with respect to $x^{\prime} \in \mathbb{R}^{n-1}$. The Fourier transform of (1.9) can be written in the form:

$$
\frac{d}{d t} \widehat{u}+\widehat{L}_{\xi^{\prime}}(t) \widehat{u}=0, t>s,\left.\quad \widehat{u}\right|_{t=s}=\widehat{u}_{0},
$$

where $\widehat{u}$ denotes the Fourier transform of $u$ in $x^{\prime}$; and $\xi^{\prime}$ is dual variable to $x^{\prime}$. For each $\xi^{\prime} \in \mathbb{R}^{n-1}$ and for all $t \geq s$ there exists a unique evolution operator $\widehat{U}_{\xi^{\prime}}(t, s)$ for $(1.11)$.

Since $\widehat{L}_{\xi^{\prime}}(t)$ is $T$-time periodic, the spectrum of $\widehat{U}_{\xi^{\prime}}(T, 0)$ plays an important role in the study of large time behavior. It was shown in [1] that the spectrum of $\widehat{U}_{\xi^{\prime}}(T, 0)$ satisfies the following inclusion

$$
\sigma\left(\widehat{U}_{\xi^{\prime}}(T, 0)\right) \subseteq \begin{cases}\left\{e^{\lambda_{\xi^{\prime}} T}\right\} \cup\left\{|\lambda|<q_{1}\right\} & \left(\left|\xi^{\prime}\right|<r\right) \\ \left\{|\lambda|<q_{1}\right\} & \left(\left|\xi^{\prime}\right| \geq r\right)\end{cases}
$$

for a constant $0<q_{1}<1$ and $0<r \ll 1$. Here, $e^{\lambda_{\xi^{\prime}} T}$ is the simple eigenvalue of $\widehat{U}_{\xi^{\prime}}(T, 0)$ and $\lambda_{\xi^{\prime}}=$ $-i \kappa_{0} \xi_{1}-\kappa_{1} \xi_{1}^{2}-\kappa^{\prime \prime}\left|\xi^{\prime \prime}\right|^{2}+O\left(\left|\xi^{\prime}\right|^{3}\right)$ with $\kappa_{0} \in \mathbb{R}, \kappa_{1}>0, \kappa^{\prime \prime}>0$ and $\xi^{\prime}={ }^{T}\left(\xi_{1}, \xi^{\prime \prime}\right), \xi^{\prime \prime}=\left(\xi_{2}, \ldots, \xi_{n-1}\right)$.

In [2] spectral properties of $\widehat{U}_{\xi^{\prime}}(t, s)$ were investigated for $\left|\xi^{\prime}\right|<r$ by using the Floquet theory. A family $\{\mathbb{P}(t)\}_{t \in \mathbb{R}}$ of bounded projections on $L^{2}(\Omega)$ was constructed to represent $\mathbb{P}(t) U(t, s)$ as

$$
\mathbb{P}(t) U(t, s)=\mathscr{Q}(t) e^{(t-s) \Lambda} \mathscr{P}(s) .
$$

Here, $e^{t \Lambda}=\mathscr{F}^{-1} \widehat{\chi}_{1} e^{\lambda_{\xi^{\prime}} t} \mathscr{F}$ with frequency cut off function $\widehat{\chi}_{1}: \widehat{\chi}_{1}\left(\xi^{\prime}\right)=1\left(\left|\xi^{\prime}\right|<r\right), \widehat{\chi}_{1}\left(\xi^{\prime}\right)=0\left(\left|\xi^{\prime}\right| \geq r\right)$, and $\mathscr{Q}(t)=\mathscr{F}^{-1} \widehat{\chi}_{1} \widehat{\mathscr{Q}}_{\xi^{\prime}}(t) \mathscr{F}$ and $\mathscr{P}(t)=\mathscr{F}^{-1} \widehat{\chi}_{1} \widehat{\mathscr{P}}_{\xi^{\prime}}(t) \mathscr{F}$ with

$$
\widehat{\mathscr{Q}}_{\xi^{\prime}}(t): \mathbb{C} \rightarrow L^{2}(0,1) \text { and } \widehat{\mathscr{P}}_{\xi^{\prime}}(t): L^{2}(0,1) \rightarrow \mathbb{C},
$$

expanded as

$$
\begin{aligned}
& \widehat{\mathscr{Q}}_{\xi^{\prime}}(t)=\mathscr{Q}^{(0)}(t)+i \xi^{\prime} \cdot \mathscr{Q}^{(1)}(t)+O\left(\left|\xi^{\prime}\right|^{2}\right), \\
& \widehat{\mathscr{P}}_{\xi^{\prime}}(t)=\mathscr{P}^{(0)}+i \xi^{\prime} \cdot \mathscr{P}^{(1)}(t)+O\left(\left|\xi^{\prime}\right|^{2}\right),
\end{aligned}
$$


for $\left|\xi^{\prime}\right| \leq r$, where $\mathscr{Q}^{(0)}(t) \sigma=\sigma u^{(0)}(\cdot, t)(\sigma \in \mathbb{C}), u^{(0)}(\cdot, t)=u^{(0)}\left(x_{n}, t\right)$ is a function $T$-periodic in $t$ and $\mathscr{P}^{(0)} u=[\phi]\left(u={ }^{T}(\phi, w) \in L^{2}(0,1)\right)$. One consequence of $(1.12)$ is that

$$
\begin{gathered}
\left\|\partial_{x^{\prime}}^{k} \partial_{x_{n}}^{l} \mathbb{P}(t) U(t, s) u_{0}\right\|_{L^{2}(\Omega)} \leq C(1+t-s)^{-\frac{n-1}{4}-\frac{k}{2}}\left\|u_{0}\right\|_{L^{1}(\Omega)}, \\
\left\|(I-\mathbb{P}(t)) U(t, s) u_{0}\right\|_{H^{1}(\Omega)} \leq e^{-d(t-s)}\left(\left\|u_{0}\right\|_{H^{1} \times L^{2}}+\left\|\partial_{x^{\prime}} w_{0}\right\|_{L^{2}}\right), \\
\left\|\partial_{x^{\prime}}^{k} \partial_{x_{n}}^{l}\left(\mathbb{P}(t) U(t, s) u_{0}-\sigma_{t, s}\left[u_{0}\right] u^{(0)}(t)\right)\right\|_{L^{2}(\Omega)} \leq C(t-s)^{-\frac{n-1}{4}-\frac{1}{2}-\frac{k}{2}}\left\|u_{0}\right\|_{L^{1}(\Omega)},
\end{gathered}
$$

for $t-s \geq T, s \geq 0 ; k=0,1, \ldots, l=0, \ldots, m$ for $m \geq 2$. Here, $\sigma_{t, s}\left[u_{0}\right]=\sigma_{t, s}\left(x^{\prime}\right)\left[u_{0}\right]$ is a function whose Fourier transform in $x^{\prime}$ is given by

$$
\mathscr{F}\left(\sigma_{t, s}\left[u_{0}\right]\right)=e^{-\left(i \kappa_{0} \xi_{1}+\kappa_{1} \xi_{1}^{2}+\kappa^{\prime \prime}\left|\xi^{\prime \prime}\right|^{2}\right)(t-s)}\left[\widehat{\phi}_{0}\left(\xi^{\prime}\right)\right]
$$

where $\left[\widehat{\phi}_{0}\left(\xi^{\prime}\right)\right]$ is a quantity given by

$$
\left[\widehat{\phi}_{0}\left(\xi^{\prime}\right)\right]=\int_{0}^{1} \widehat{\phi}_{0}\left(\xi^{\prime}, x_{n}\right) d x_{n}
$$

with $\widehat{\phi}_{0}$ being the Fourier transform of $\phi_{0}$ in $x^{\prime}$ and $\kappa_{0} \in \mathbb{R}, \kappa_{1}>0, \kappa^{\prime \prime}>0$ are positive constants depending on $\rho_{*}, l, V, \mu, \mu^{\prime}$ and $\widetilde{P}^{\prime}\left(\rho_{*}\right)$.

Another consequence of $(1.12)$ is that if $u(t)$ is a solution of

$$
\partial_{t} u+L(t) u=f,\left.u\right|_{t=0}=u_{0}
$$

then $\mathbb{P}(t) u(t)$ is represented as

$$
\mathbb{P}(t) u(t)=\mathscr{Q}(t)\left(e^{t \Lambda \mathscr{P}}(0) u_{0}+\int_{0}^{t} e^{(t-z) \Lambda} \mathscr{P}(z) f(z) d z\right) .
$$

In this paper we show the following results. Let $u_{0}$ be sufficiently small in $H^{m}(\Omega) \cap L^{1}(\Omega)$ for a given $m \geq[n / 2]+1$; and let $u_{0}$ satisfy a suitable compatibility condition, then there exists unique solution $u(t)$ of (1.5)-(1.8) in $C\left([0, \infty) ; H^{m}(\Omega)\right)$, provided that $R e \ll 1, M a \ll 1$ and $\left|1-\rho_{p}\right|_{C^{m+1}([0,1])} \ll 1$. Furthermore, $u(t)$ satisfies

$$
\left\|\partial_{x^{\prime}}^{k} u(t)\right\|_{L^{2}(\Omega)} \leq O\left(t^{-\frac{n-1}{4}-\frac{k}{2}}\right), k=0,1
$$

as $t \rightarrow \infty$.

In the case $n=2$, we show that the asymptotic leading term of perturbation $u(t)$ is described by a solution of one-dimensional viscous Burgers equation, i.e.,

$$
\left\|u(t)-\left(\sigma u^{(0)}\right)(t)\right\|_{2}=O\left(t^{-\frac{3}{4}+\delta}\right), \forall \delta>0,
$$

as $t \rightarrow \infty$. Here, $u^{(0)}=u^{(0)}\left(x_{2}, t\right)$ is a given time-periodic function; and $\sigma=\sigma\left(x_{1}, t\right)$ is function satisfying

$$
\partial_{t} \sigma-\kappa_{1} \partial_{x_{1}}^{2} \sigma+\kappa_{0} \partial_{x_{1}} \sigma+\omega_{0} \partial_{x_{1}}\left(\sigma^{2}\right)=0,\left.\sigma\right|_{t=0}=\int_{0}^{1} \phi_{0}\left(x_{1}, x_{2}\right) d x_{2},
$$

with constants $\kappa_{0} \in \mathbb{R}, \kappa_{1}>0$ being the same ones as those in $\lambda_{\xi_{1}}$ and $\omega_{0} \in \mathbb{R}$ determined by the nonlinearity $\boldsymbol{F}$.

In the case $n \geq 3$, we show that the asymptotic leading term of $u(t)$ is the same one as for the linearized problem and thus it is given by $n$-1-dimensional heat equation with convective term, i.e.,

$$
\left\|u(t)-\left(\sigma u^{(0)}\right)(t)\right\|_{2}=O\left(t^{-\frac{n-1}{4}-\frac{1}{2}} \eta_{n}(t)\right)
$$

as $t \rightarrow \infty$. Here, $\sigma=\sigma\left(x^{\prime}, t\right)$ is function satisfying

$$
\partial_{t} \sigma-\kappa_{1} \partial_{x_{1}}^{2} \sigma-\kappa^{\prime \prime} \Delta^{\prime \prime} \sigma+\kappa_{0} \partial_{x_{1}} \sigma=0,\left.\sigma\right|_{t=0}=\int_{0}^{1} \phi_{0}\left(x^{\prime}, x_{n}\right) d x_{n},
$$

with constants $\kappa_{0} \in \mathbb{R}, \kappa_{1}, \kappa^{\prime \prime}>0$ being the same ones as those in $\lambda_{\xi^{\prime}}$; where $\Delta^{\prime \prime}=\partial_{x_{2}}^{2}+\cdots+\partial_{x_{n-1}}^{2}$; and $\eta_{n}(t)=\log (1+t)$ when $n=3$ and $\eta_{n}(t)=1$ when $n \geq 4$.

The proof of the main results is given by a combination of various estimates for $\mathbb{P}(t) U(t, s)$ mentioned above and a variant of Matsumura-Nishida energy method $([6,7]$, cf. [12]). We decompose the solution $u(t)$ 
of (1.5)-(1.8) into the $\mathbb{P}(t)$-part and $(I-\mathbb{P}(t))$-part. Considering the $\mathbb{P}(t)$-part, we represent $\mathbb{P}(t) u(t)$ as in (1.13) with $f={ }^{T}\left(f^{0}, \boldsymbol{f}\right)$ being the nonlinearity given in (1.5) and (1.6). We then combine various estimates on $\mathbb{P}(t)$ and $\mathbb{P}(t) U(t, s)$ to obtain the necessary estimates on $\mathbb{P}(t) u(t)$. On the other hand, $(I-\mathbb{P}(t)) u(t)$ can be estimated by a variant of Matsumura-Nishida energy method as in the case of the stationary parallel flow ([7]). However, in contrast to [7], the linearized operator has time-dependent coefficients. Therefore a modification of the argument in [7] is needed for the time-periodic case to aquire the necessary energy estimate. It is worth mentioning that in the case $n=2$ the asymptotic leading part of $u(t)$ is not described by the linearized problem due to the quadratic nonlinearities $-\operatorname{div}(\phi w), \frac{\nu \phi}{\gamma^{2} \rho_{p}^{2}}\left(-\partial_{x_{n}}^{2} w^{1}+\frac{\partial_{x_{n}}^{2} v_{p}^{1}}{\rho_{p} \gamma^{2}} \phi\right)$ and $-\frac{1}{2 \gamma^{4} \rho_{p}} \partial_{x_{n}}\left(P^{\prime \prime}\left(\rho_{p}\right) \phi^{2}\right)$. This leads to the 1-dimensional Burgers equation (1.14).

Our result is an extension of previous results on the stationary case $[5,6,7,11]$ to the case of time-periodic external force and time-periodic boundary conditions.

Structure of this paper is the following. In Section 2 we introduce basic notations that are used throughout the paper. In Section 3 we state the main results. In Section 4 we present the results on spectral properties of the linearized problem obtained in [2]. In Section 5 we introduce decomposition of solution $u(t)$ to $(1.5)-(1.8)$ based on the spectral properties of $L(t)$ introduced in Section 4. Moreover, we prove the a priori estimate using the estimates on $\mathbb{P}(t) u(t)$ and $(I-\mathbb{P}(t)) u(t)$ from subsequent sections 6,7 and 8 . In Section 6 we show estimate for $\mathbb{P}(t) u(t)$ using properties of $\mathbb{P}(t)$ and $\mathbb{P}(t) U(t, s)$. In Section 7 we obtain estimate on $(I-\mathbb{P}(t)) u(t)$ using energy method. In Section 8 we estimate the nonlinearities $f^{0}$ and $\boldsymbol{f}$. Finally, in Section 9 we prove the asymptotic behavior of solutions.

\section{Notation}

In this section we introduce some notations which are used throughout the paper. For a domain $E$ we denote by $L^{p}(E)$ the usual Lebesgue space on $E$ and its norm is denoted by $\|\cdot\|_{L^{p}(E)}$ for $1 \leq p \leq \infty$. Let $k$ be a nonnegative integer. $H^{k}(E)$ denotes the $k$-th order $L^{2}$ Sobolev space on $E$ with norm $\|\cdot\|_{H^{k}(E)}$. $C_{0}^{k}(E)$ stands for the set of all $C^{k}$ functions which have compact support in $E$. We denote by $H_{0}^{1}(E)$ the completion of $C_{0}^{1}(E)$ in $H^{1}(E)$.

We simply denote by $L^{p}(E)$ (resp., $H^{k}(E)$ ) the set of all vector fields $w={ }^{T}\left(w^{1}, \ldots, w^{n}\right)$ on $E$ with $w^{j} \in L^{p}(E)$ (resp., $\left.H^{k}(E)\right), j=1, \ldots, n$, and its norm is also denoted by $\|\cdot\|_{L^{p}(E)}$ (resp., $\left.\|\cdot\|_{H^{k}(E)}\right)$. For $u=$ $T^{T}(\phi, w)$ with $\phi \in H^{k}(E)$ and $w={ }^{T}\left(w^{1}, \ldots, w^{n}\right) \in H^{l}(E)$, we define $\|u\|_{H^{k}(E) \times H^{l}(E)}$ by $\|u\|_{H^{k}(E) \times H^{l}(E)}=$ $\|\phi\|_{H^{k}(E)}+\|w\|_{H^{l}(E)}$. When $k=l$, we simply write $\|u\|_{H^{k}(E) \times H^{k}(E)}=\|u\|_{H^{k}(E)}$.

In the case $E=\Omega$ we abbreviate $L^{p}(\Omega)$ (resp., $H^{k}(\Omega)$ ) as $L^{p}$ (resp., $H^{k}$ ). In particular, we denote the norm of $L^{p}$ (resp., $\left.H^{k}\right)$ by $\|\cdot\|_{p}$ (resp., $\left.\|\cdot\|_{H^{k}}\right)$.

In the case $E=(0,1)$ we denote the norm $|\cdot|_{L^{2}(0,1)}\left(\right.$ resp., $\left.|\cdot|_{H^{k}(0,1)}\right)$ by $|\cdot|_{2}\left(\right.$ resp., $\left.|\cdot|_{H^{k}}\right)$.

The inner product of $L^{2}$ is denoted by

$$
(f, g)=\int_{\Omega} f(x) g(x) d x, \quad f, g \in L^{2} .
$$

Furthermore, we introduce a weighted inner product $\langle\cdot, \cdot\rangle_{\Omega}$ defined by

$$
\left\langle u_{1}, u_{2}\right\rangle_{\Omega}=\int_{\Omega} \phi_{1} \phi_{2} \frac{P^{\prime}\left(\rho_{p}\right)}{\gamma^{4} \rho_{p}} d x+\int_{\Omega} w_{1} w_{2} \rho_{p} d x
$$

for $u_{j}={ }^{T}\left(\phi_{j}, w_{j}\right) \in L^{2}, j=1,2$; and for $u_{j}={ }^{T}\left(\phi_{j}, w_{j}\right) \in L^{2}(0,1), j=1,2$, we also define $\left\langle u_{1}, u_{2}\right\rangle$ by

$$
\left\langle u_{1}, u_{2}\right\rangle=\int_{0}^{1} \phi_{1} \bar{\phi}_{2} \frac{P^{\prime}\left(\rho_{p}\right)}{\gamma^{4} \rho_{p}} d x_{n}+\int_{0}^{1} w_{1} \bar{w}_{2} \rho_{p} d x_{n} .
$$

Here, $\bar{g}$ denotes the complex conjugate of $g$.

Furthermore, for $f \in L^{1}(0,1)$ we denote the mean value of $f$ in $(0,1)$ by $[f]$ :

$$
[f]=(f, 1)=\int_{0}^{1} f\left(x_{n}\right) d x_{n} .
$$

For $u={ }^{T}(\phi, w) \in L^{1}(0,1)$ with $w={ }^{T}\left(w^{1}, \ldots, w^{n}\right)$ we define $[u]$ by

$$
[u]=[\phi]+\left[w^{1}\right]+\cdots+\left[w^{n}\right]
$$

We often write $x \in \Omega$ as 


$$
x={ }^{T}\left(x^{\prime}, x_{n}\right), x^{\prime}={ }^{T}\left(x_{1}, \ldots, x_{n-1}\right) \in \mathbb{R}^{n-1} .
$$

Partial derivatives of function $u$ in $x, x^{\prime}, x_{n}$ and $t$ are denoted by $\partial_{x} u, \partial_{x^{\prime}} u, \partial_{x_{n}} u$ and $\partial_{t} u$, respectively. We also write higher order partial derivatives of $u$ in $x$ as $\partial_{x}^{k} u=\left(\partial_{x}^{\alpha} u ;|\alpha|=k\right)$; by $\Delta^{\prime}=\sum_{i=1}^{n-1} \partial_{x_{i}}^{2}$, $\nabla^{\prime}=\left(\partial_{x_{1}}, \ldots, \partial_{x_{n-1}}\right)$ and $\operatorname{div}^{\prime}=\nabla^{\prime}$. we denote the Lapacian, gradient and divergence with respect to $x^{\prime}$, respectively.

We denote $k \times k$ identity matrix by $I_{k}$. In particular, when $k=n+1$, we simply write $I$ for $I_{n+1}$. We define $(n+1) \times(n+1)$ diagonal matrices $Q_{j}, Q^{\prime}$ and $\widetilde{Q}$ by

$$
Q_{j}=\operatorname{diag}(0, \ldots, 0, \underbrace{1}_{j \text {-th }}, 0, \ldots, 0), \quad j=0,1, \ldots, n,
$$

and

$$
Q^{\prime}=\operatorname{diag}(0,1, \ldots, 1,0), \widetilde{Q}=\operatorname{diag}(0,1, \ldots, 1) .
$$

We then have for $u={ }^{T}(\phi, w) \in \mathbb{R}^{n+1}, w={ }^{T}\left(w^{1}, \ldots, w^{n}\right)={ }^{T}\left(w^{\prime}, w^{n}\right)$,

$$
Q_{0} u=\left(\begin{array}{c}
\phi \\
0
\end{array}\right), \quad Q_{j} u=\left(\begin{array}{c}
0 \\
w^{j} \\
0
\end{array}\right), \quad Q_{n} u=\left(\begin{array}{c}
0 \\
0 \\
w^{n}
\end{array}\right), \quad Q^{\prime} u=\left(\begin{array}{c}
0 \\
w^{\prime} \\
0
\end{array}\right), \quad \widetilde{Q} u=\left(\begin{array}{c}
0 \\
w
\end{array}\right) .
$$

We denote $\boldsymbol{e}_{1}^{\prime}={ }^{T}(1,0, \ldots, 0) \in \mathbb{R}^{n-1}$. We note that

$$
\left[Q_{0} u\right]=[\phi] \text { for } u={ }^{T}(\phi, w) .
$$

For a function $f=f\left(x^{\prime}\right)\left(x^{\prime} \in \mathbb{R}^{n-1}\right)$, we denote its Fourier transform by $\widehat{f}$ or $\mathscr{F} f$ :

$$
\widehat{f}\left(\xi^{\prime}\right)=(\mathscr{F} f)\left(\xi^{\prime}\right)=\int_{\mathbb{R}^{n-1}} f\left(x^{\prime}\right) e^{-i \xi^{\prime} \cdot x^{\prime}} d x^{\prime} .
$$

The inverse Fourier transform is denoted by $\mathscr{F}^{-1}$ :

$$
\left(\mathscr{F}^{-1} f\right)\left(x^{\prime}\right)=(2 \pi)^{-(n-1)} \int_{\mathbb{R}^{n-1}} f\left(\xi^{\prime}\right) e^{i \xi^{\prime} \cdot x^{\prime}} d \xi^{\prime} .
$$

For closed linear operator $A$ in $X$ we denote the spectrum of $A$ by $\sigma(A)$. We denote the set of all bounded linear operators from $X_{0}$ into itself by $L\left(X_{0}\right)$ and denote the norm by $|\cdot|_{L\left(X_{0}\right)}$. For operators $A, B$ we denote $[A, B]$ the commutator, i.e., $[A, B]=A B-B A$. For time interval $[a, b] \subset \mathbb{R}$, we denote the usual Bochner spaces by $L^{2}(a, b ; X), H^{m}(a, b ; X)$, etc., where $X$ denotes a Banach space.

Definition 2.1 For a domain $E$ we define the following function spaces:

$$
X_{0}=H^{1}(0,1) \times L^{2}(0,1), \quad H_{*}^{j}(E)=\left\{\begin{array}{cc}
H^{-1}(E)=\left(H_{0}^{1}\right)^{*}(E) & \text { for } j=-1, \\
L^{2}(E) & \text { for } j=0, \\
H^{j}(E) \cap H_{0}^{1}(E) & \text { for } j \geq 1 .
\end{array}\right.
$$

Definition 2.2 We introduce the following norms:

$$
\begin{gathered}
\llbracket f(t) \rrbracket_{k}=\left(\sum_{j=0}^{\left[\frac{k}{2}\right]}\left\|\partial_{t}^{j} f(t)\right\|_{H^{k-2 j}}\right)^{\frac{1}{2}}, \\
\|\mid D f(t)\| \|_{k}= \begin{cases}\left\|\partial_{x} f(t)\right\|_{2} & \text { for } k=0, \\
\left(\llbracket \partial_{x} f(t) \rrbracket_{k}^{2}+\llbracket \partial_{t} f(t) \rrbracket_{k-1}^{2}\right)^{\frac{1}{2}} & \text { for } k \geq 1 .\end{cases}
\end{gathered}
$$

Remark 2.3 Let us note that

$$
\left\||D v \||_{m-1} \leq 2 \llbracket v \rrbracket_{m} \text { and } \llbracket v \rrbracket_{m} \leq\right\| v\left\|_{2}+\right\| D v \|_{m-1},
$$

for $\llbracket v \rrbracket_{m}<\infty$. 
Definition 2.4 Let $m \geq[n / 2]+1$. For $\tau>0$ we define a function space $Z^{m}(\tau)$ by

$$
Z^{m}(\tau)=\left\{u \in \bigcap_{j=0}^{\left[\frac{m}{2}\right]} C^{j}\left([0, \tau] ; H^{m-2 j}\right),\|u\|_{Z^{m}(\tau)}<\infty\right\}
$$

where

$$
\|u\|_{Z^{m}(\tau)}=\sup _{0 \leq z \leq \tau} \llbracket u(z) \rrbracket_{m}+\left(\int_{0}^{\tau}\|\mid D w(z)\| \|_{m}^{2} d z\right)^{\frac{1}{2}}
$$

\section{$3 \quad$ Main results}

In this section we state the main results of this paper.

In the whole article we assume the following regularity for $\widetilde{\boldsymbol{g}}$ and $\widetilde{V}^{1}$.

Assumptions 3.1 For a given integer $m \geq[n / 2]+1$ assume that $\widetilde{\boldsymbol{g}}={ }^{T}\left(\widetilde{g}^{1}\left(\widetilde{x}_{n}, \widetilde{t}\right), 0, \ldots, 0, \widetilde{g}^{n}\left(\widetilde{x}_{n}\right)\right)$ and $\widetilde{V}^{1}(\widetilde{t})$ belong to the following spaces:

$$
\widetilde{g}^{n} \in C^{m+1}[0, \ell]
$$

and

$$
\begin{gathered}
\widetilde{g}^{1} \in \bigcap_{j=0}^{\left[\frac{m+1}{2}\right]} C_{p e r}^{j}\left([0, \tau] ; H^{m+1-2 j}(0, \ell)\right), \\
\widetilde{V}^{1} \in C_{p e r}^{\left[\frac{m+2}{2}\right]}([0, \tau]) .
\end{gathered}
$$

Furthermore, we assume

$$
\widetilde{P}(\cdot) \in C^{m+2}(\mathbb{R})
$$

It is straightforward that $\boldsymbol{g}$ and $V^{1}$ belong to similar spaces as $\widetilde{\boldsymbol{g}}$ and $\widetilde{V}^{1}$.

Under Assumptions 3.1 one can see that flow $u_{p}$ has the following properties (see [1]).

Proposition 3.2 There exists $\delta_{0}>0$ such that if

$$
\nu\left|g^{n}\right|_{C^{m+1}([0,1])} \leq \delta_{0},
$$

then the following assertions hold true(see [1]). The flow $u_{p}={ }^{T}\left(\rho_{p}\left(x_{n}\right), v_{p}\left(x_{n}, t\right)\right)$ exists and under Assumptions 3.1, it satisfies

$$
v_{p} \in \bigcap_{j=0}^{\left[\frac{m+3}{2}\right]} C_{p e r}^{j}\left(J_{T} ; H^{m+3-2 j}(0,1)\right), \quad \rho_{p} \in C^{m+2}[0,1],
$$

and

$$
0<\rho_{1} \leq \rho_{p}\left(x_{n}\right) \leq \rho_{2}, \int_{0}^{1} \rho_{p}\left(x_{n}\right) d x_{n}=1, v_{p}\left(x_{n}, t\right)={ }^{T}\left(v_{p}^{1}\left(x_{n}, t\right), 0\right)
$$

with

$$
\begin{gathered}
P^{\prime}(\rho)>0 \text { for } \rho_{1} \leq \rho \leq \rho_{2}, \\
\left|1-\rho_{p}\right|_{C^{k+1}([0,1])} \leq \frac{C}{\gamma^{2}} \nu\left(\left|P^{\prime \prime}\right|_{C^{k-1}\left(\rho_{1}, \rho_{2}\right)}+\left|g^{n}\right|_{C^{k}([0,1])}\right), \quad k=1, \ldots, m+1 \\
\left|P^{\prime}\left(\rho_{p}\right)-\gamma^{2}\right|_{C^{0}([0,1])} \leq \frac{C}{\gamma^{2}} \nu\left|g^{n}\right|_{C^{0}([0,1])}
\end{gathered}
$$

for some constants $0<\rho_{1}<1<\rho_{2}$. 
First, let us introduce the local existence result. To do so, we rewrite (1.5)-(1.8) in the form

$$
\begin{gathered}
\partial_{t} \phi+v \cdot \nabla \phi=-\gamma^{2} w \cdot \nabla \rho_{p}-\rho \operatorname{div} w \\
\rho \partial_{t} w-\nu \Delta w-\widetilde{\nu} \nabla \operatorname{div} w=-\frac{\nu}{\gamma^{2} \rho_{p}} \partial_{x_{n}}^{2} v_{p} \phi-\nabla\left(P(\rho)-P\left(\rho_{p}\right)\right)-\rho(v \cdot \nabla v), \\
\left.w\right|_{\partial \Omega}=0, \\
\left.(\phi, w)\right|_{t=0}=\left(\phi_{0}, w_{0}\right),
\end{gathered}
$$

where $\rho=\rho_{p}+\gamma^{-2} \phi$ and $v=v_{p}+w$.

Next, let us mention the compatibility condition for $u_{0}={ }^{T}\left(\phi_{0}, w_{0}\right)$. We look for a solution $u={ }^{T}(\phi, w)$ of (3.2)-(3.5) in $\bigcap_{j=0}^{\left[\frac{m}{2}\right]} C^{j}\left([0, \infty) ; H^{m-2 j}\right)$ satisfying $\int_{0}^{t}\left\|\partial_{x} w(z)\right\|_{H^{m}}^{2} d z<\infty$ for all $t \geq 0$ with $m \geq[n / 2]+1$. Therefore, we need to require the compatibility condition for the initial value $u_{0}={ }^{T}\left(\phi_{0}, w_{0}\right)$, which is formulated as follows.

Let $u={ }^{T}(\phi, w)$ be a smooth solution of (3.2)-(3.5). Then $\partial_{t}^{j} u={ }^{T}\left(\partial_{t}^{j} \phi, \partial_{t}^{j} w\right), j \geq 1$ is inductively determined by

$$
\partial_{t}^{j} \phi=-v \cdot \nabla \partial_{t}^{j-1} \phi-\rho \operatorname{div} \partial_{t}^{j-1} w-\gamma^{2} \partial_{t}^{j-1} w \cdot \nabla \rho_{p}-\left\{\left[\partial_{t}^{j-1}, v \cdot \nabla\right] \phi+\left[\partial_{t}^{j-1}, \rho\right] \operatorname{div} w\right\},
$$

and

$$
\begin{aligned}
\partial_{t}^{j} w=-\rho^{-1}\left\{-\nu \Delta \partial_{t}^{j-1} w-\widetilde{\nu} \nabla \operatorname{div} \partial_{t}^{j-1} w\right. & \left.+P^{\prime}(\rho) \nabla \partial_{t}^{j-1} \rho\right\}-\rho^{-1}\left\{\gamma^{-2}\left[\partial_{t}^{j-1}, \phi\right] \partial_{t} w+\left[\partial_{t}^{j-1}, P^{\prime}(\rho)\right] \nabla \rho\right\} \\
& -\rho^{-1}\left\{\frac{\nu}{\gamma^{2} \rho_{p}} \partial_{t}^{j-1}\left(\partial_{x_{n}}^{2} v_{p} \phi\right)-\partial_{t}^{j-1} \nabla P\left(\rho_{p}\right)\right\}-\rho^{-1} \partial_{t}^{j-1}(\rho(v \cdot \nabla v))
\end{aligned}
$$

From these relations we see that $\left.\partial_{t}^{j} u\right|_{t=0}=\left.{ }^{T}\left(\partial_{t}^{j} \phi, \partial_{t}^{j} w\right)\right|_{t=0}$ is inductively given by $u_{0}={ }^{T}\left(\phi_{0}, w_{0}\right)$ in the following way:

where

$$
\left.\partial_{t}^{j} u\right|_{t=0}=\left.{ }^{T}\left(\partial_{t}^{j} \phi, \partial_{t}^{j} w\right)\right|_{t=0}={ }^{T}\left(\phi_{j}, w_{j}\right)=u_{j}
$$

$$
\phi_{j}=-v_{0} \cdot \nabla \phi_{j-1}-\rho_{0} \operatorname{div} w_{j-1}-\gamma^{2} w_{j-1} \cdot \nabla \rho_{p}-\sum_{l=1}^{j-1}\left(\begin{array}{c}
j-1 \\
l
\end{array}\right)\left\{v_{l} \cdot \nabla \phi_{j-1-l}+\gamma^{-2} \phi_{l} \operatorname{div} w_{j-1}\right\}
$$

and

$$
\begin{gathered}
w_{j}=-\rho_{0}^{-1}\left\{-\nu \Delta w_{j-1}-\widetilde{\nu} \nabla \operatorname{div} w_{j-1}+P^{\prime}\left(\rho_{0}\right) \nabla \rho_{j-1}\right\}-\rho_{0}^{-1} \sum_{l=1}^{j-1}\left(\begin{array}{c}
j-1 \\
l
\end{array}\right)\left\{\gamma^{-2} \phi_{l} w_{j-l}\right. \\
\left.+a_{l}\left(\phi_{0} ; \phi_{1}, \ldots, \phi_{l}\right) \nabla \rho_{j-1-l}\right\}-\rho_{0}^{-1} \frac{\nu}{\gamma^{2} \rho_{p}} \sum_{l=0}^{j-1}\left(\begin{array}{c}
j-1 \\
l
\end{array}\right) \partial_{t}^{j-1-l} \partial_{x_{n}}^{2} v_{p}(0) \phi_{l}+\delta_{1 j} \rho_{0}^{-1} \nabla P\left(\rho_{p}\right) \\
-\rho_{0}^{-1} G_{j-1}\left(\phi_{0}, w_{0}, \partial_{x} w_{0} ; \phi_{1}, \ldots, \phi_{j-1}, w_{1}, \ldots, w_{j-1}, \partial_{x} w_{1}, \ldots, \partial_{x} w_{j-1}\right),
\end{gathered}
$$

with $v_{l}=\partial_{t}^{l} v_{p}(0)+w_{l}, \rho_{l}=\delta_{1 l} \rho_{p}+\gamma^{-2} \phi_{l}$; and $a_{l}\left(\phi_{0} ; \phi_{1}, \ldots, \phi_{l}\right)$ is certain polynomial in $\phi_{1}, \ldots, \phi_{l}$; and analogously. Here, $\delta_{1 j}$ denotes the Kronecker's delta.

By the boundary condition $\left.w\right|_{\partial \Omega}=0$ in (3.4), we necessarily have $\left.\partial_{t}^{j} w\right|_{\partial \Omega}=0$, and hence,

$$
\left.w_{j}\right|_{\partial \Omega}=0
$$

Assume that $u={ }^{T}(\phi, w)$ is a solution of $(3.2)-(3.5)$ in $\bigcap_{j=0}^{\left[\frac{m}{2}\right]} C^{j}\left(\left[0, \tau_{0}\right] ; H^{m-2 j}\right)$ for some $\tau_{0}>0$. Then from above observation, we need the regularity $u_{j}={ }^{T}\left(\phi_{j}, w_{j}\right) \in H^{m-2 j} \times H^{m-2 j}$ for $j=1, \ldots,[m / 2]$, which, indeed follows from the fact that $u_{0}={ }^{T}\left(\phi_{0}, w_{0}\right) \in H^{m}$ with $m \geq[n / 2]+1$. Furthermore, it is necessary to require that $u_{0}={ }^{T}\left(\phi_{0}, w_{0}\right)$ satisfies the $\widehat{m}$-th order compatibility condition:

$$
w_{j} \in H_{0}^{1} \text { for } j=0, \ldots, \widehat{m}=\left[\frac{m-1}{2}\right] \text {. }
$$

Now, we can apply local solvability result obtained in [8] to show the following assertion. 
Proposition 3.3 Let $n \geq 2, m$ be an integer satisfying $m \geq[n / 2]+1$ and $M>0$. Assume that $u_{0}=$ $T\left(\phi_{0}, w_{0}\right) \in H^{m}$ satisfies the following conditions:

(a) $\left\|u_{0}\right\|_{H^{m}} \leq M$ and $u_{0}$ satisfies the $\widehat{m}$-th compatibility condition,

(b) $-\frac{\gamma^{2}}{4} \rho_{1} \leq \phi_{0}$

Then there exists a positive number $\tau_{0}$ depending on $M$ and $\rho_{1}$ such that problem (3.2)-(3.5) has a unique solution $u(t)$ on $\left[0, \tau_{0}\right]$ satisfying $u(t) \in Z^{m}\left(\tau_{0}\right)$.

Remark 3.4 It is straightforward to see that solution $u(t)$ of $(3.2)-(3.5)$ is a solution of (1.5)-(1.8). Condition (b) in previous proposition assures that $\gamma^{-2} \phi_{0}+\rho_{p}>\frac{3}{4} \rho_{1}$.

We are in a position to state our main results of this paper.

Theorem 3.5 Suppose that $n=2$ and let $m$ be an integer satisfying $m \geq 2$. There are positive numbers $\nu_{0}$ and $\gamma_{0}$ such that if $\nu \geq \nu_{0}$ and $\gamma^{2} /(\nu+\widetilde{\nu}) \geq \gamma_{0}^{2}$ then the following assertions hold true.

There is a positive number $\varepsilon_{0}$ such that if $u_{0}={ }^{T}\left(\phi_{0}, w_{0}\right) \in H^{m} \cap L^{1}$ satisfies the $\widehat{m}$-th compatibility condition and $\left\|u_{0}\right\|_{H^{m} \cap L^{1}} \leq \varepsilon_{0}$, then there exists a unique global solution $u(t)={ }^{T}(\phi(t), w(t))$ of $(1.5)-(1.8)$ with $n=2$ in $\bigcap_{j=0}^{\left[\frac{m}{2}\right]} C^{j}\left([0, \infty) ; H^{m-2 j}\right)$ which satisfies

$$
\begin{gathered}
\left\|\partial_{x^{\prime}}^{k} u(t)\right\|_{2}=O\left(t^{-\frac{1}{4}-\frac{k}{2}}\right), k=0,1, \\
\left\|u(t)-\left(\sigma u^{(0)}\right)(t)\right\|_{2}=O\left(t^{-\frac{3}{4}+\delta}\right), \quad \forall \delta>0,
\end{gathered}
$$

as $t \rightarrow \infty$. Here, $u^{(0)}=u^{(0)}\left(x_{2}, t\right)$ is function given in Lemma 4.9 below; $\sigma=\sigma\left(x_{1}, t\right)$ is function satisfying

$$
\partial_{t} \sigma-\kappa_{1} \partial_{x_{1}}^{2} \sigma+\kappa_{0} \partial_{x_{1}} \sigma+\omega_{0} \partial_{x_{1}}\left(\sigma^{2}\right)=0,\left.\sigma\right|_{t=0}=\int_{0}^{1} \phi_{0}\left(x_{1}, x_{2}\right) d x_{2}
$$

with given constants $\kappa_{0}, \omega_{0} \in \mathbb{R}, \kappa_{1}>0$.

Theorem 3.6 Suppose that $n \geq 3$ and let $m$ be an integer satisfying $m \geq[n / 2]+1$. There are positive numbers $\nu_{0}$ and $\gamma_{0}$ such that if $\nu \geq \nu_{0}$ and $\gamma^{2} /(\nu+\widetilde{\nu}) \geq \gamma_{0}^{2}$ then the following assertions hold true.

There is a positive number $\varepsilon_{0}$ such that if $u_{0}={ }^{T}\left(\phi_{0}, w_{0}\right) \in H^{m} \cap L^{1}$ satisfies the $\widehat{m}$-th compatibility condition and $\left\|u_{0}\right\|_{H^{m} \cap L^{1}} \leq \varepsilon_{0}$, then there exists a unique global solution $u(t)={ }^{T}(\phi(t), w(t))$ of $(1.5)-(1.8)$ in $\bigcap_{j=0}^{\left[\frac{m}{2}\right]} C^{j}\left([0, \infty) ; H^{m-2 j}\right)$ which satisfies

$$
\begin{gathered}
\left\|\partial_{x^{\prime}}^{k} u(t)\right\|_{2}=O\left(t^{-\frac{n-1}{4}-\frac{k}{2}}\right), k=0,1, \\
\left\|u(t)-\left(\sigma u^{(0)}\right)(t)\right\|_{2}=O\left(t^{-\frac{n-1}{4}-\frac{1}{2}} \eta_{n}(t)\right),
\end{gathered}
$$

as $t \rightarrow \infty$. Here, $\sigma=\sigma\left(x^{\prime}, t\right)$ is function satisfying

$$
\partial_{t} \sigma-\kappa_{1} \partial_{x_{1}}^{2} \sigma-\kappa^{\prime \prime} \Delta^{\prime \prime} \sigma+\kappa_{0} \partial_{x_{1}} \sigma=0,\left.\sigma\right|_{t=0}=\int_{0}^{1} \phi_{0}\left(x^{\prime}, x_{n}\right) d x_{n},
$$

with given constants $\kappa_{0} \in \mathbb{R}, \kappa_{1}, \kappa^{\prime \prime}>0$; where $\Delta^{\prime \prime}=\partial_{x_{2}}^{2}+\cdots+\partial_{x_{n-1}}^{2}$; and $\eta_{n}(t)=\log (1+t)$ when $n=3$ and $\eta_{n}(t)=1$ when $n \geq 4$.

As in $[8,12]$, the global existence result in Theorem 3.5 and Theorem 3.6 is proved by combining the local existence and the a priori estimate. Next we introduce the a priori estimate.

Proposition 3.7 Let $n \geq 2$ and $m$ be an integer satisfying $m \geq[n / 2]+1$. There are positive numbers $\nu_{0}$ and $\gamma_{0}$ such that if $\nu \geq \nu_{0}$ and $\gamma^{2} /(\nu+\widetilde{\nu}) \geq \gamma_{0}^{2}$, then the following assertion holds true.

There exists number $\varepsilon_{1}>0$ such that if solution $u(t)$ of $(1.5)-(1.8)$ is in $Z^{m}(\tau)$ and $u(t)$ satisfies $\left\|u_{0}\right\|_{H^{m} \cap L^{1}} \leq \varepsilon_{1}$, then there holds the estimate

$$
\llbracket u(t) \rrbracket_{m}^{2} \leq C_{1}\left\|u_{0}\right\|_{H^{m} \cap L^{1}}^{2}
$$

for a constant $C_{1}>0$ independent of $\tau$. 
Remark 3.8 In the proof of Proposition 3.7 we use the estimate (3.1) with $k=m$ only, i.e.,

$$
\left|1-\rho_{p}\right|_{C^{m+1}([0,1])} \leq \frac{C}{\gamma^{2}} \nu\left(\left|P^{\prime \prime}\right|_{C^{m-1}\left(\rho_{1}, \rho_{2}\right)}+\left|g^{n}\right|_{C^{m}([0,1])}\right) .
$$

Moreover, we require the boundedness of $\rho_{p}$ in $C^{m+1}([0,1])$ only.

The global existence of the solution $u(t)$ follows from Proposition 3.3 and the a priori estimate in Proposition 3.7 in standard manner as follows.

Proof of global existence. Let $n \geq 2$ and let us fix $m \geq[n / 2]+1$ and $\nu \geq \nu_{0}, \gamma^{2} /(\nu+\widetilde{\nu}) \geq \gamma_{0}^{2}$ such that Proposition 3.7 holds true.

Since $m \geq[n / 2]+1$ we have the Sobolev inequality

$$
\|f\|_{\infty} \leq C_{S}\|f\|_{H^{m}}, \text { for any } f \in H^{m}(\Omega)
$$

Let us define $\varepsilon_{0}>0$ as

$$
\varepsilon_{0}=\min \left\{\varepsilon_{1}, \frac{\gamma^{2}}{4 C_{S}} \rho_{1}, \frac{\varepsilon_{1}}{\sqrt{C_{1}}}, \frac{\gamma^{2}}{4 C_{S} \sqrt{C_{1}}} \rho_{1}\right\} .
$$

Here, $\varepsilon_{1}$ and $C_{1}$ are given by Proposition 3.7.

Let $\left\|u_{0}\right\|_{H^{m} \cap L^{1}} \leq \varepsilon_{0}$ satisfies $\widehat{m}$-th compatibility condition. It is easy to see that such $u_{0}$ satisfies conditions (a), (b) of Proposition 3.3 and therefore, there exits $\tau_{0}>0$, which is determined by $\varepsilon_{1}$, such that the problem (1.5)-(1.8) has a unique solution $u(\cdot) \in Z^{m}\left(\tau_{0}\right)$.

Since $\varepsilon_{0} \leq \varepsilon_{1}$ we see from Proposition 3.7 that $u(t)$ satisfies

$$
\llbracket u\left(\tau_{0}\right) \rrbracket_{m}^{2} \leq C_{1}\left\|u_{0}\right\|_{H^{m} \cap L^{1}}^{2} \leq \min \left\{\varepsilon_{1}^{2},\left(\frac{\gamma^{2}}{4 C_{S}} \rho_{1}\right)^{2}\right\} .
$$

Thus, $\left\|u\left(\tau_{0}\right)\right\|_{H^{m}} \leq \varepsilon_{1}$ and $u\left(\tau_{0}\right)$ satisfies conditions (a) and (b) of Proposition 3.3. Hence, there exists unique extension of solution $u(t)$ of $(1.5)-(1.8)$ on $\left[\tau_{0}, 2 \tau_{0}\right]$ and we get

$$
u(\cdot) \in Z^{m}\left(2 \tau_{0}\right) .
$$

It is straightforward to see that we can use Proposition 3.7 again, to obtain estimate $(3.11)$ for $u\left(2 \tau_{0}\right)$, which enables us to extend solution $u(t)$ on $\left[0,3 \tau_{0}\right]$. By repeating this procedure the existence on $[0, \infty)$ is showed. This concludes the proof.

Proposition 3.7 together with $L^{2}$-decay estimates (3.6) and (3.8) are proved in Sections 4-8. The asymptotic behavior, i.e., (3.7) and (3.9), is proved in Section 9.

\section{Spectral properties of the linearized operator}

Let us write (1.5)-(1.8) in the form

$$
\begin{gathered}
\partial_{t} u+L(t) u=\boldsymbol{F}, \\
\left.w\right|_{\delta \Omega}=0,\left.u\right|_{t=0}=u_{0} .
\end{gathered}
$$

Here, $u={ }^{T}(\phi, w) ; \boldsymbol{F}={ }^{T}\left(f^{0}, \boldsymbol{f}\right)$ with $\boldsymbol{f}={ }^{T}\left(f^{1}, \cdots, f^{n}\right)$ is the nonlinearity; and $L(t)$ is the operator given in $(1.10)$

In this section we introduce the spectral properties of the linearized problem, i.e., $(4.1)$ with $\boldsymbol{F}=0$. These results were established in [2]. At the end of this section we show regularity improvements for $\phi$.

Now, let us consider the linearized problem

$$
\partial_{t} u+L(t) u=0, t>s,\left.w\right|_{x_{n}=0,1}=0,\left.u\right|_{t=s}=u_{0}
$$

We introduce space $Z_{s}$ defined by

$Z_{s}=\left\{u={ }^{T}(\phi, w) ; \phi \in C_{l o c}\left([s, \infty) ; H^{1}\right), \partial_{x^{\prime}}^{\alpha^{\prime}} w \in C_{l o c}\left([s, \infty) ; L^{2}\right) \cap L_{l o c}^{2}\left([s, \infty) ; H_{0}^{1}\right)\left(\left|\alpha^{\prime}\right| \leq 1\right), w \in C_{l o c}\left((s, \infty) ; H_{0}^{1}\right)\right\}$.

In [1] we showed that for any initial data $u_{0}={ }^{T}\left(\phi_{0}, w_{0}\right)$ satisfying $u_{0} \in H^{1} \cap L^{2}$ with $\partial_{x^{\prime}} w_{0} \in L^{2}$ there exists a unique solution $u(t)$ of linear problem $(4.2)$ in $Z_{s}$. We denote $U(t, s)$ the evolution operator for $(4.2)$ given by

$$
u(t)=U(t, s) u_{0} .
$$


To investigate problem (4.2) we consider the Fourier transform of (4.2). We thus obtain

$$
\frac{d}{d t} \widehat{u}+\widehat{L}_{\xi^{\prime}}(t) \widehat{u}=0, t>s,\left.\quad \widehat{u}\right|_{t=s}=\widehat{u}_{0} .
$$

Here $\widehat{\phi}=\widehat{\phi}\left(\xi^{\prime}, x_{n}, t\right)$ and $\widehat{w}=\widehat{w}\left(\xi^{\prime}, x_{n}, t\right)$ are the Fourier transforms of $\phi=\phi\left(x^{\prime}, x_{n}, t\right)$ and $w=w\left(x^{\prime}, x_{n}, t\right)$ in $x^{\prime} \in \mathbb{R}^{n-1}$ with $\xi^{\prime} \in \mathbb{R}^{n-1}$ being the dual variable; $\widehat{L}_{\xi^{\prime}}(t)$ is an operator on $X_{0}$ with domain $D\left(\widehat{L}_{\xi^{\prime}}(t)\right)=$ $H^{1}(0,1) \times H_{*}^{2}(0,1)$, which takes the form

$$
\begin{gathered}
\widehat{L}_{\xi^{\prime}}(t)=\left(\begin{array}{ccc}
i \xi_{1} v_{p}^{1}(t) & i \gamma^{2} \rho_{p}^{T} \xi^{\prime} & \gamma^{2} \partial_{x_{n}}\left(\rho_{p} \cdot\right) \\
i \xi^{\prime} \frac{P^{\prime}\left(\rho_{p}\right)}{\gamma^{2} \rho_{p}} & \frac{\nu}{\rho_{p}}\left(\left|\xi^{\prime}\right|^{2}-\partial_{x_{n}}^{2}\right) I_{n-1}+\frac{\widetilde{\nu}}{\rho_{p}} \xi^{\prime T} \xi^{\prime} & -i \frac{\widetilde{\nu}}{\rho_{p}} \xi^{\prime} \partial_{x_{n}} \\
\partial_{x_{n}}\left(\frac{P^{\prime}\left(\rho_{p}\right)}{\gamma^{2} \rho_{p}}\right) & -i \frac{\widetilde{\nu}}{\rho_{p}} \xi^{\prime} \partial_{x_{n}} & \frac{\nu}{\rho_{p}}\left(\left|\xi^{\prime}\right|^{2}-\partial_{x_{n}}^{2}\right)-\frac{\widetilde{\nu}}{\rho_{p}} \partial_{x_{n}}^{2}
\end{array}\right) \\
+\left(\begin{array}{ccc}
0 & 0 & 0 \\
\frac{\nu}{\gamma^{2} \rho_{p}^{2}}\left(\partial_{x_{n}}^{2} v_{p}^{1}(t)\right) \boldsymbol{e}_{1}^{\prime} & i \xi_{1} v_{p}^{1}(t) I_{n-1} & \partial_{x_{n}}\left(v_{p}^{1}(t)\right) \boldsymbol{e}_{1}^{\prime} \\
0 & 0 & i \xi_{1} v_{p}^{1}(t)
\end{array}\right) .
\end{gathered}
$$

Let us note that $\widehat{L}_{\xi^{\prime}}(t)$ is sectorial uniformly with respect to $t \in \mathbb{R}$ for each $\xi^{\prime} \in \mathbb{R}^{n-1}$. As for the evolution operator $\widehat{U}_{\xi^{\prime}}(t, s)$ for $(4.3)$ we have the following results.

Lemma 4.1 For each $\xi^{\prime} \in \mathbb{R}^{n-1}$ and for all $t \geq s$ there exists unique evolution operator $\widehat{U}_{\xi^{\prime}}(t, s)$ for $(4.3)$ that satisfies

$$
\left|\widehat{L}_{\xi^{\prime}}(t) \widehat{U}_{\xi^{\prime}}(t, s)\right|_{L\left(X_{0}\right)} \leq C_{t_{1} t_{2}}, t_{1} \leq s<t \leq t_{2} .
$$

Furthermore, for $u_{0} \in X_{0}, f \in C^{\alpha}\left([s, \infty) ; X_{0}\right), \alpha \in(0,1]$ there exists unique classical solution $u$ of inhomogeneous problem

$$
\frac{d}{d t} u+\widehat{L}_{\xi^{\prime}}(t) u=f, t>s,\left.\quad u\right|_{t=s}=u_{0},
$$

satisfying $u \in C_{l o c}\left([s, \infty) ; X_{0}\right) \cap C^{1}\left(s, \infty ; X_{0}\right) \cap C\left(s, \infty ; H^{1}(0,1) \times H_{*}^{2}(0,1)\right)$; and the solution $u$ is given by

$$
u(t)=(\phi(t), w(t))=\widehat{U}_{\xi^{\prime}}(t, s) u_{0}+\int_{s}^{t} \widehat{U}_{\xi^{\prime}}(t, z) f(z) d z .
$$

Next, let us introduce adjoint problem to

$$
\partial_{t} u+\widehat{L}_{\xi^{\prime}}(t) u=0, t>s,\left.\quad u\right|_{t=s}=u_{0}
$$

Lemma 4.2 For each $\xi^{\prime} \in \mathbb{R}^{n-1}$ and for all $s \leq t$ there exists unique evolution operator $\widehat{U}_{\xi^{\prime}}^{*}(s, t)$ for adjoint problem

$$
-\partial_{s} u+\widehat{L}_{\xi^{\prime}}^{*}(s) u=0, s<t,\left.u\right|_{s=t}=u_{0},
$$

on $X_{0}$. Here, $\widehat{L}_{\xi^{\prime}}^{*}(s)$ is an operator on $X_{0}$ with domain $D\left(\widehat{L}_{\xi^{\prime}}^{*}(s)\right)=H^{1}(0,1) \times H_{*}^{2}(0,1)$, which takes the form

$$
\begin{gathered}
\widehat{L}_{\xi^{\prime}}^{*}(s)=\left(\begin{array}{rcc}
-i \xi_{1} v_{p}^{1}(s) & -i \gamma^{2} \rho_{p}^{T} \xi^{\prime} & -\gamma^{2} \partial_{x_{n}}\left(\rho_{p} \cdot\right) \\
-i \xi^{\prime} \frac{P^{\prime}\left(\rho_{p}\right)}{\gamma^{2} \rho_{p}} & \frac{\nu}{\rho_{p}}\left(\left|\xi^{\prime}\right|^{2}-\partial_{x_{n}}^{2}\right) I_{n-1}+\frac{\widetilde{\nu}}{\rho_{p}} \xi^{\prime T} \xi^{\prime} & -i \frac{\widetilde{\nu}}{\rho_{p}} \xi^{\prime} \partial_{x_{n}} \\
-\partial_{x_{n}}\left(\frac{P^{\prime}\left(\rho_{p}\right)}{\gamma^{2} \rho_{p}} \cdot\right) & -i \frac{\widetilde{\nu}}{\rho_{p}} \xi^{\prime} \partial_{x_{n}} & \frac{\nu}{\rho_{p}}\left(\left|\xi^{\prime}\right|^{2}-\partial_{x_{n}}^{2}\right)-\frac{\widetilde{\nu}}{\rho_{p}} \partial_{x_{n}}^{2}
\end{array}\right) \\
+\left(\begin{array}{ccc}
0 & \frac{\nu \gamma^{2}}{P^{\prime}\left(\rho_{p}\right)}\left(\partial_{x_{n}}^{2} v_{p}^{1}(s)\right)^{T} \boldsymbol{e}_{1}^{\prime} & 0 \\
0 & -i \xi_{1} v_{p}^{1}(s) I_{n-1} & 0 \\
0 & \partial_{x_{n}}\left(v_{p}^{1}(s)\right)^{T} \boldsymbol{e}_{1}^{\prime} & -i \xi_{1} v_{p}^{1}(s)
\end{array}\right)
\end{gathered}
$$

Moreover, $\widehat{L}_{\xi^{\prime}}^{*}(s)$ satisfies $\left\langle\widehat{L}_{\xi^{\prime}}(s) u, v\right\rangle=\left\langle u, \widehat{L}_{\xi^{\prime}}^{*}(s) v\right\rangle$ for $s \in \mathbb{R}$ and $u, v \in H^{1} \times H_{*}^{2}$ and 


$$
\left|\widehat{L}_{\xi^{\prime}}^{*}(s) \widehat{U}_{\xi^{\prime}}^{*}(s, t)\right|_{L\left(X_{0}\right)} \leq C_{t_{1} t_{2}}, t_{1} \leq s<t \leq t_{2} .
$$

Furthermore, for $u_{0} \in X_{0}, f \in C^{\alpha}\left((-\infty, t] ; X_{0}\right), \alpha \in(0,1]$ there exists unique classical solution $u$ of inhomogeneous problem

$$
-\frac{d}{d s} u+\widehat{L}_{\xi^{\prime}}^{*}(s) u=f, s<t,\left.\quad u\right|_{s=t}=u_{0}
$$

satisfying $u \in C_{l o c}\left((-\infty, t] ; X_{0}\right) \cap C^{1}\left(-\infty, t ; X_{0}\right) \cap C\left(-\infty, t ; H^{1}(0,1) \times H_{*}^{2}(0,1)\right)$; and the solution $u$ is given by

$$
u(s)=(\phi(s), w(s))=\widehat{U}_{\xi^{\prime}}^{*}(s, t) u_{0}+\int_{s}^{t} \widehat{U}_{\xi^{\prime}}^{*}(s, z) f(z) d z .
$$

Note that $\widehat{U}_{\xi^{\prime}}(t, s)$ and $\widehat{U}_{\xi^{\prime}}^{*}(s, t)$ are defined for all $t \geq s$ and

$$
\widehat{U}_{\xi^{\prime}}(t+T, s+T)=\widehat{U}_{\xi^{\prime}}(t, s), \widehat{U}_{\xi^{\prime}}^{*}(s+T, t+T)=\widehat{U}_{\xi^{\prime}}^{*}(s, t) .
$$

Lemma 4.3 There exist positive numbers $\nu_{1}$ and $\gamma_{1}$ such that if $\nu \geq \nu_{1}$ and $\gamma^{2} /(\nu+\widetilde{\nu}) \geq \gamma_{1}^{2}$ then there exists $r_{0}>0$ such that for each $\xi^{\prime}$ with $\left|\xi^{\prime}\right| \leq r_{0}$ there hold the following statements.

(i) The spectrum of operator $\widehat{U}_{\xi^{\prime}}(T, 0)$ on $H^{1}(0,1) \times H_{0}^{1}(0,1)$ satisfies

$$
\sigma\left(\widehat{U}_{\xi^{\prime}}(T, 0)\right) \subset\left\{\mu_{\xi^{\prime}}\right\} \cup\left\{\mu:|\mu| \leq q_{0}\right\},
$$

with constant $q_{0}<\operatorname{Re} \mu_{\xi^{\prime}}<1$. Here, $\mu_{\xi^{\prime}}=e^{\lambda_{\xi^{\prime}} T}$ is simple eigenvalue of $\widehat{U}_{\xi^{\prime}}(T, 0)$ and $\lambda_{\xi^{\prime}}$ has an expansion

$$
\lambda_{\xi^{\prime}}=-i \kappa_{0} \xi_{1}-\kappa_{1} \xi_{1}^{2}-\kappa^{\prime \prime}\left|\xi^{\prime \prime}\right|^{2}+O\left(\left|\xi^{\prime}\right|^{3}\right),
$$

where $\kappa_{0} \in \mathbb{R}$ and $\kappa_{1}>0, \kappa^{\prime \prime}>0$.

Moreover, let $\widehat{\Pi}_{\xi^{\prime}}$ denote the eigenprojection associated with $\mu_{\xi^{\prime}}$. There holds

$$
\left|\widehat{U}_{\xi^{\prime}}(t, s)\left(I-\widehat{\Pi}_{\xi^{\prime}}\right) u\right|_{H^{1}} \leq C e^{-d(t-s)}\left|\left(I-\widehat{\Pi}_{\xi^{\prime}}\right) u\right|_{X_{0}},
$$

for $u \in X_{0}$ and $T \leq t-s$. Here, $d$ is a positive constant depending on $r_{0}$.

(ii) The spectrum of operator $\widehat{U}_{\xi^{\prime}}^{*}(0, T)$ on $H^{1}(0,1) \times H_{0}^{1}(0,1)$ satisfies

$$
\sigma\left(\widehat{U}_{\xi^{\prime}}^{*}(0, T)\right) \subset\left\{\bar{\mu}_{\xi^{\prime}}\right\} \cup\left\{\mu:|\mu| \leq q_{0}\right\} .
$$

Here, $\bar{\mu}_{\xi^{\prime}}$ is simple eigenvalue of $\widehat{U}_{\xi^{\prime}}^{*}(0, T)$.

Furthermore, let $\widehat{\Pi}_{\xi^{\prime}}^{*}$ denote the eigenprojection associated with $\bar{\mu}_{\xi^{\prime}}$. There holds

$$
\left\langle\widehat{\Pi}_{\xi^{\prime}} u, v\right\rangle=\left\langle u, \widehat{\Pi}_{\xi^{\prime}}^{*} v\right\rangle,
$$

for $u, v \in X_{0}$.

Next, we introduce Floquet theory.

Definition 4.4 Let $k=1,2, \ldots$. Let us define spaces $Y_{\text {per }}^{k}$ as

$$
\begin{gathered}
Y_{\text {per }}^{1}=L_{\text {per }}^{2}\left([0, T] ; X_{0}\right), \\
Y_{\text {per }}^{k}=\bigcap_{j=0}^{\left[\frac{k}{2}\right]} H_{p e r}^{j}\left([0, T] ; H^{k-2 j}(0,1) \times H^{k-1-2 j}(0,1)\right), \text { for } k \geq 2 .
\end{gathered}
$$

Here, for Banach space $X$ and $j=0, \ldots$ spaces $L_{p e r}^{2}([0, T] ; X)$ and $H_{p e r}^{j}([0, T] ; X)$ consist of functions from $L^{2}([0, T] ; X)$ and $H^{j}([0, T] ; X)$, respectively, that are restrictions of $T$-periodic functions. 
Definition 4.5 We define operator $B_{\xi^{\prime}}$ on space $Y_{\text {per }}^{1}$ with domain

$$
D\left(B_{\xi^{\prime}}\right)=H_{p e r}^{1}\left([0, T] ; X_{0}\right) \cap L_{p e r}^{2}\left([0, T] ; H^{1}(0,1) \times H_{*}^{2}(0,1)\right),
$$

in the following way

$$
B_{\xi^{\prime}} v=\partial_{t} v+\widehat{L}_{\xi^{\prime}}(\cdot) v
$$

for $v \in D\left(B_{\xi^{\prime}}\right)$. Moreover, we define formal adjoint operator $B_{\xi^{\prime}}^{*}$ with respect to inner product $\frac{1}{T} \int_{0}^{T}\langle\cdot, \cdot\rangle d t$ as

$$
B_{\xi^{\prime}}^{*} v=-\partial_{t} v+\widehat{L}_{\xi^{\prime}}^{*}(\cdot) v
$$

for $v \in D\left(B_{\xi^{\prime}}^{*}\right)=D\left(B_{\xi^{\prime}}\right)$.

Remark 4.6 Operators $B_{\xi^{\prime}}$ and $B_{\xi^{\prime}}^{*}$ are closed, densely defined on $Y_{\text {per }}^{1}$ for each fixed $\xi^{\prime} \in \mathbb{R}^{n-1}$.

Definition 4.7 Let $k \geq 1$. We say that $u={ }^{T}(\phi, w)$ is $k$-regular function on time interval $[a, b]$ whenever

$$
\begin{gathered}
u \in \bigcap_{j=0}^{\left[\frac{k}{2}\right]} C^{j}\left([a, b] ;\left(H^{k-2 j} \times H_{*}^{k-2 j}\right)(\Omega)\right), \\
\phi \in \bigcap_{j=0}^{\left[\frac{k}{2}\right]} H^{j+1}\left(a, b ; H^{k-2 j}(\Omega)\right), w \in \bigcap_{j=0}^{\left[\frac{k+1}{2}\right]} H^{j}\left(a, b ; H_{*}^{k+1-2 j}(\Omega)\right) .
\end{gathered}
$$

Lemma 4.8 There exist positive numbers $\nu_{2} \geq \nu_{1}$ and $\gamma_{2} \geq \gamma_{1}$ such that if $\nu \geq \nu_{2}$ and $\gamma^{2} /(\nu+\widetilde{\nu}) \geq \gamma_{2}^{2}$ then there exists $0<r_{1} \leq 1$ such that for each $\left|\xi^{\prime}\right| \leq r_{1}$ there hold the following statements.

(i) Let $1 \leq k \leq m+1$. There exists $q_{1}>0$ such that spectrum of operator $B_{\xi^{\prime}}$ on $Y_{p e r}^{k}$ satisfies

$$
\sigma\left(B_{\xi^{\prime}}\right) \subset\left\{-\lambda_{\xi^{\prime}}\right\} \cup\left\{\lambda: \operatorname{Re} \lambda \geq q_{1}\right\}
$$

with $0 \leq-\operatorname{Re} \lambda_{\xi^{\prime}} \leq \frac{1}{2} q_{1}$ uniform for all $k$. Here, $-\lambda_{\xi^{\prime}}$ is simple eigenvalue of $B_{\xi^{\prime}}$.

(ii) Let $1 \leq k \leq m+1$. Spectrum of operator $B_{\xi^{\prime}}^{*}$ on $Y_{p e r}^{k}$ satisfies

$$
\sigma\left(B_{\xi^{\prime}}^{*}\right) \subset\left\{-\bar{\lambda}_{\xi^{\prime}}\right\} \cup\left\{\lambda: \operatorname{Re} \lambda \geq q_{1}\right\}
$$

Here, $-\bar{\lambda}_{\xi^{\prime}}$ is simple eigenvalue of $B_{\xi^{\prime}}^{*}$.

(iii) There exist $u_{\xi^{\prime}}$ and $u_{\xi^{\prime}}^{*}$ eigenfunctions associated with $-\lambda_{\xi^{\prime}}$ and $-\bar{\lambda}_{\xi^{\prime}}$, respectively, with the following properties:

$$
\begin{gathered}
\left\langle u_{\xi^{\prime}}(t), u_{\xi^{\prime}}^{*}(t)\right\rangle=1, \\
u_{\xi^{\prime}}(t)=u^{(0)}(t)+i \xi^{\prime} \cdot u^{(1)}(t)+\left|\xi^{\prime}\right|^{2} u^{(2)}\left(\xi^{\prime}, t\right), \\
u_{\xi^{\prime}}^{*}(t)=u^{*(0)}+i \xi^{\prime} \cdot u^{*(1)}(t)+\left|\xi^{\prime}\right|^{2} u^{*(2)}\left(\xi^{\prime}, t\right),
\end{gathered}
$$

for $t \in \mathbb{R}$. Here, all functions

$$
u_{\xi^{\prime}}, u_{\xi^{\prime}}^{*}, u^{(0)}, u^{(0) *}, u^{(1)}, u^{(1) *}, u^{(2)}\left(\xi^{\prime}\right), u^{(2) *}\left(\xi^{\prime}\right),
$$

are T-periodic in $t, m+1$-regular on $[0, T]$ and we have estimate

$$
\sup _{z \in J_{T}} \sum_{j=0}^{\left[\frac{m+1}{2}\right]}\left|\partial_{z}^{j} u(z)\right|_{H^{m+1-2 j}}^{2}+\int_{0}^{T} \sum_{j=0}^{\left[\frac{m}{2}\right]}\left|\partial_{z}^{j+1} u\right|_{H^{m+1-2 j} \times H^{m-2 j}}^{2}+\left|\partial_{z}^{\left[\frac{m+3}{2}\right]} Q_{0} u\right|_{2}^{2}+|u|_{H^{m+1} \times H^{m+2}}^{2} d z \leq C
$$

for $u \in\left\{u_{\xi^{\prime}}, u_{\xi^{\prime}}^{*}, u^{(2)}\left(\xi^{\prime}\right), u^{(2) *}\left(\xi^{\prime}\right)\right\}$ and a constant $C>0$ depending on $r_{1}$. 
Let us introduce more properties of $u^{(0)}$.

Lemma 4.9 Function $u^{(0)}(t)$ satisfies $\partial_{t} u^{(0)}+\widehat{L}_{0}(t) u^{(0)}=0$ and $u^{(0)}(t)=u^{(0)}(t+T)$ for all $t \in \mathbb{R}$. Function $u^{(0)}(t)$ is given as

$$
u^{(0)}\left(x_{n}, t\right)={ }^{T}\left(\phi^{(0)}\left(x_{n}\right), w^{(0), 1}\left(x_{n}, t\right), 0\right)
$$

Here,

$$
\begin{gathered}
\phi^{(0)}\left(x_{n}\right)=\alpha_{0} \frac{\gamma^{2} \rho_{p}\left(x_{n}\right)}{P^{\prime}\left(\rho_{p}\left(x_{n}\right)\right)}, \quad \alpha_{0}=\left[\frac{\gamma^{2} \rho_{p}}{P^{\prime}\left(\rho_{p}\right)}\right]^{-1}, \\
w^{(0), 1}\left(x_{n}, t\right)=-\frac{1}{\gamma^{2}} \int_{-\infty}^{t} e^{-(t-s) \nu A} \nu \frac{\alpha_{0} \gamma^{2}}{P^{\prime}\left(\rho_{p}\right) \rho_{p}}\left(\partial_{x_{n}}^{2} v_{p}^{1}(s)\right) d s,
\end{gathered}
$$

where $A$ denotes the uniformly elliptic operator on $L^{2}(0,1)$ with domain $D(A)=\left(H^{2} \cap H_{0}^{1}\right)(0,1)$ and

$$
A v=-\frac{1}{\rho_{p}\left(x_{n}\right)} \partial_{x_{n}}^{2} v
$$

for $v \in D(A)$. Moreover, function $w^{(0), 1}$ satisfies

for all $t \in \mathbb{R}$ and

$$
\partial_{t} w^{(0), 1}(t)-\frac{\nu}{\rho_{p}\left(x_{n}\right)} \partial_{x_{n}}^{2} w^{(0), 1}(t)=-\frac{\nu}{\gamma^{2}} \frac{\alpha_{0} \gamma^{2}}{P^{\prime}\left(\rho_{p}\right) \rho_{p}}\left(\partial_{x_{n}}^{2} v_{p}^{1}(t)\right),
$$

$$
\left\|w^{(0), 1}(t)\right\|_{C^{m+1}(\Omega)}=O\left(\frac{1}{\gamma^{2}}\right)
$$

In the rest of this section we assume that $\nu \geq \nu_{2}$ and $\gamma^{2} /(\nu+\widetilde{\nu}) \geq \gamma_{2}^{2}$.

Definition 4.10 We define $\widehat{\chi}_{1}$ by

$$
\widehat{\chi}_{1}\left(\xi^{\prime}\right)= \begin{cases}1, & \left|\xi^{\prime}\right|<r_{1} \\ 0, & \left|\xi^{\prime}\right| \geq r_{1}\end{cases}
$$

for $\xi^{\prime} \in \mathbb{R}^{n-1}$.

Now, we introduce time-periodic operators based on $u_{\xi^{\prime}}$ and $u_{\xi^{\prime}}^{*}$.

Definition 4.11 We define operators $\mathscr{P}(t): L^{2}(\Omega) \rightarrow L^{2}\left(\mathbb{R}^{n-1}\right)$ by

$$
\begin{gathered}
\mathscr{P}(t) u=\mathscr{F}^{-1}\left\{\widehat{\mathscr{P}}_{\xi^{\prime}}(t) \widehat{u}\right\}, \\
\widehat{\mathscr{P}}_{\xi^{\prime}}(t) \widehat{u}=\widehat{\chi}_{1}\left\langle\widehat{u}, u_{\xi^{\prime}}^{*}(t)\right\rangle,
\end{gathered}
$$

for $u \in L^{2}$ and $t \in[0, \infty)$.

We define operators $\mathscr{Q}(t): L^{2}\left(\mathbb{R}^{n-1}\right) \rightarrow L^{2}(\Omega)$ by

$$
\begin{gathered}
\mathscr{Q}(t) \sigma=\mathscr{F}^{-1}\left\{\widehat{\mathscr{Q}}_{\xi^{\prime}}(t) \widehat{\sigma}\right\}, \\
\widehat{\mathscr{Q}}_{\xi^{\prime}}(t) \widehat{\sigma}=\widehat{\chi}_{1} u_{\xi^{\prime}}(\cdot, t) \widehat{\sigma},
\end{gathered}
$$

for $t \in[0, \infty)$ and multiplier $\Lambda: L^{2}\left(\mathbb{R}^{n-1}\right) \rightarrow L^{2}\left(\mathbb{R}^{n-1}\right)$ by

$$
\Lambda \sigma=\mathscr{F}^{-1}\left\{\widehat{\chi}_{1} \lambda_{\xi^{\prime}} \widehat{\sigma}\right\}
$$

for $\sigma \in L^{2}\left(\mathbb{R}^{n-1}\right)$.

Moreover, we define projections $\mathbb{P}(t)$ and $\mathbb{P}^{*}(t)$ on $L^{2}(\Omega)$ as

$$
\begin{gathered}
\mathbb{P}(t) u=\mathscr{F}^{-1}\left\{\widehat{\chi}_{1}\left\langle u, u_{\xi^{\prime}}^{*}(t)\right\rangle u_{\xi^{\prime}}(\cdot, t)\right\}=\mathscr{Q}(t) \mathscr{P}(t) u, \\
\mathbb{P}^{*}(t) u=\mathscr{F}^{-1}\left\{\widehat{\chi}_{1}\left\langle u, u_{\xi^{\prime}}(t)\right\rangle u_{\xi^{\prime}}^{*}(\cdot, t)\right\},
\end{gathered}
$$

for $t \in[0, \infty)$ and $u \in L^{2}$.

We define projection $\Pi^{(0)}(t)$ on $L^{2}(\Omega)$ as

$$
\Pi^{(0)}(t) u=\left[Q_{0} u\right] u^{(0)}(t),
$$

for $t \in[0, \infty)$ and $u \in L^{2}$. 
In terms of $P(t)$ we have the following decomposition of $U(t, s)$.

Lemma 4.12 $\mathbb{P}(t)$ and $\mathbb{P}^{*}(t)$ satisfies the following:

(i)

$$
\mathbb{P}(t)\left(\partial_{t}+L(t)\right) u(t)=\left(\partial_{t}+L(t)\right) \mathbb{P}(t) u(t)=\mathscr{Q}(t)\left[\left(\partial_{t}-\Lambda\right) \mathscr{P}(t) u(t)\right],
$$

for $u \in L^{2}\left([0, T] ; H^{1} \times H_{*}^{2}\right) \cap H^{1}\left([0, T] ; L^{2}\right)$.

(ii)

If $u \in L^{1}$, then

$$
\mathbb{P}(t) U(t, s)=U(t, s) \mathbb{P}(s)=\mathscr{Q}(t) e^{(t-s) \Lambda} \mathscr{P}(s) .
$$

$$
\left\|\partial_{t}^{j} \partial_{x^{\prime}}^{k} \partial_{x_{n}}^{l} \mathbb{P}(t) U(t, s) u\right\|_{2} \leq C(1+t-s)^{-\frac{n-1}{4}-\frac{k}{2}}\|u\|_{1},
$$

for $0 \leq 2 j+l \leq m+1, k=0, \ldots$.

(iii) For $u, v \in L^{2}$ there holds

$$
\langle\mathbb{P}(t) u, v\rangle=\left\langle u, \mathbb{P}^{*}(t) u\right\rangle
$$

If $u \in L^{2}$, then

$$
\left\|\partial_{t}^{j} \partial_{x^{\prime}}^{k} \partial_{x_{n}}^{l}\left(\mathbb{P}^{*}(t) u\right)\right\|_{2} \leq C\|u\|_{2},
$$

for $0 \leq 2 j+l \leq m+1, k=0,1, \ldots$.

(iv) $(I-\mathbb{P}(t)) U(t, s)=U(t, s)(I-\mathbb{P}(s))$ satisfies

$$
\|(I-\mathbb{P}(t)) U(t, s) u\|_{H^{1}} \leq C e^{-d(t-s)}\left(\|u\|_{H^{1} \times L^{2}}+\left\|\partial_{x^{\prime}} w\right\|_{2}\right),
$$

for $t-s \geq T$. Here $d$ is a positive constant.

Next, let us show the asymptotic properties of $U(t, s)$. First, let us define a semigroup $\mathscr{H}(t)$ on $L^{2}\left(\mathbb{R}^{n-1}\right)$ associated with a linear heat equation with a convective term:

$$
\partial_{t} \sigma-\kappa_{1} \partial_{x_{1}}^{2} \sigma-\kappa^{\prime \prime} \Delta^{\prime \prime} \sigma+\kappa_{0} \partial_{x_{1}} \sigma=0
$$

Definition 4.13 We define operator $\mathscr{H}(t)$ as

$$
\mathscr{H}(t) \sigma=\mathscr{F}^{-1}\left[e^{-\left(i \kappa_{0} \xi_{1}+\kappa_{1} \xi_{1}^{2}+\kappa^{\prime \prime}\left|\xi^{\prime \prime}\right|^{2}\right) t} \widehat{\sigma}\right]
$$

for $\sigma \in L^{2}\left(\mathbb{R}^{n-1}\right)$. Here, $\kappa_{0}, \kappa_{1}$ and $\kappa^{\prime \prime}$ are given by $(4.6)$.

Lemma 4.14 There hold the following estimates for $1 \leq p \leq 2$ and $k=0,1, \ldots$

(i)

for $\sigma \in L^{p}\left(\mathbb{R}^{n-1}\right)$.

$$
\left\|\partial_{x^{\prime}}^{k}(\mathscr{H}(t) \sigma)\right\|_{L^{2}\left(\mathbb{R}^{n-1}\right)} \leq C t^{-\frac{n-1}{2}\left(\frac{1}{p}-\frac{1}{2}\right)-\frac{k}{2}}\|\sigma\|_{L^{p}\left(\mathbb{R}^{n-1}\right)}
$$

(ii) $\Lambda$ generates uniformly continuous group $\left\{e^{t \Lambda}\right\}_{t \in \mathbb{R}}$ and

$$
\left\|\partial_{x^{\prime}}^{k} e^{t \Lambda} \sigma\right\|_{L^{2}\left(\mathbb{R}^{n-1}\right)} \leq C(1+t)^{-\frac{n-1}{2}\left(\frac{1}{p}-\frac{1}{2}\right)-\frac{k}{2}}\|\sigma\|_{L^{p}\left(\mathbb{R}^{n-1}\right)},
$$

for $\sigma \in L^{p}\left(\mathbb{R}^{n-1}\right)$.

(iii) It holds the relation,

$$
\mathscr{P}(t) U(t, s)=e^{(t-s) \Lambda} \mathscr{P}(s)
$$

Set $\sigma=\left[Q_{0} u\right]$. Then

$$
\left\|\partial_{x^{\prime}}^{k}\left(e^{(t-s) \Lambda} \mathscr{P}(s) u-\mathscr{H}(t-s) \sigma\right)\right\|_{L^{2}\left(\mathbb{R}^{n-1}\right)} \leq C(t-s)^{-\frac{n-1}{2}\left(\frac{1}{p}-\frac{1}{2}\right)-\frac{k+1}{2}}\|u\|_{p},
$$

for $u \in L^{p}$. Furhermore, for any $\sigma \in L^{p}\left(\mathbb{R}^{n-1}\right)$ there holds

$$
\left\|\left(e^{(t-s) \Lambda}-\mathscr{H}(t-s)\right) \partial_{x^{\prime}}^{k} \sigma\right\|_{L^{2}\left(\mathbb{R}^{n-1}\right)} \leq C(t-s)^{-\frac{n-1}{2}\left(\frac{1}{p}-\frac{1}{2}\right)-\frac{k+1}{2}}\|\sigma\|_{L^{p}\left(\mathbb{R}^{n-1}\right)} .
$$


Next, we introduce the properties of $\mathscr{Q}(t)$ and $\mathscr{P}(t)$.

Proposition $4.15 \mathscr{Q}(t)$ has the following properties:

(i)

$$
\mathscr{Q}(t+T)=\mathscr{Q}(t), \partial_{x^{\prime}}^{k} \mathscr{Q}(t)=\mathscr{Q}(t) \partial_{x^{\prime}}^{k}
$$

(ii)

$$
\begin{gathered}
\mathscr{Q}(t) \sigma \in \bigcap_{j=0}^{\left[\frac{m+1}{2}\right]} C_{p e r}^{j}\left(J_{T} ; H^{m+1-2 j} \times H_{*}^{m+1-2 j}\right), \\
\widetilde{Q} \mathscr{Q}(t) \sigma \in \bigcap_{j=0}^{\left[\frac{m+2}{2}\right]} H_{p e r}^{j}\left(J_{T} ; H_{*}^{m+2-2 j}\right),
\end{gathered}
$$

and

$$
\left\|\partial_{t}^{j} \partial_{x^{\prime}}^{k} \partial_{x_{n}}^{l}(\mathscr{Q}(t) \sigma)\right\|_{2} \leq C\|\sigma\|_{L^{2}\left(\mathbb{R}^{n-1}\right)}, 0 \leq 2 j+l \leq m+1, k=0,1, \ldots,
$$

for $\sigma \in L^{2}\left(\mathbb{R}^{n-1}\right)$.

(iii)

$$
\left(\partial_{t}+L(t)\right)(\mathscr{Q}(t) \sigma(t))=\mathscr{Q}(t)\left(\partial_{t}-\Lambda\right) \sigma(t),
$$

for $\sigma \in H_{l o c}^{1}\left([0, \infty) ; L^{2}\left(\mathbb{R}^{n-1}\right)\right)$.

(iv) $\mathscr{Q}(t)$ is decomposed as

$$
\mathscr{Q}(t)=\mathscr{Q}^{(0)}(t)+\operatorname{div}^{\prime} \mathscr{Q}^{(1)}(t)+\Delta^{\prime} \mathscr{Q}^{(2)}(t) .
$$

Here, $\mathscr{Q}^{(0)}(t) \sigma=\left(\mathscr{F}^{-1}\left\{\widehat{\chi}_{1} \widehat{\sigma}\right\}\right) u^{(0)}(\cdot, t), \mathscr{Q}^{(1)}(t)$ and $\mathscr{Q}^{(2)}(t)$ share the same properties given in (i) and (ii) for $\mathscr{Q}(t)$.

Proposition 4.16 $\mathscr{P}(t)$ has the following properties:

(i)

$$
\mathscr{P}(t+T)=\mathscr{P}(t), \partial_{x^{\prime}}^{k} \mathscr{P}(t)=\mathscr{P}(t) \partial_{x^{\prime}}^{k}, \partial_{x_{n}} \mathscr{P}(t)=0
$$

(ii)

$$
\mathscr{P}(t) u \in \bigcap_{j=0}^{\left[\frac{m+1}{2}\right]} C_{p e r}^{j}\left(J_{T} ; H^{k}\left(\mathbb{R}^{n-1}\right)\right), \text { for all } k=0,1, \ldots
$$

and

$$
\left\|\partial_{t}^{j} \partial_{x^{\prime}}^{k}(\mathscr{P}(t) u)\right\|_{L^{2}\left(\mathbb{R}^{n-1}\right)} \leq C\|u\|_{2}, 0 \leq 2 j \leq m+1, k=0,1, \ldots
$$

for $u \in L^{2}$.

Moreover,

$$
\|\mathscr{P}(t) u\|_{L^{2}\left(\mathbb{R}^{n-1}\right)} \leq C\|u\|_{p},
$$

for $u \in L^{p}$ and $1 \leq p \leq 2$.

(iii)

$$
\mathscr{P}(t)\left(\partial_{t}+L(t)\right) u(t)=\left(\partial_{t}-\Lambda\right)(\mathscr{P}(t) u(t))
$$

for $u \in L_{l o c}^{2}\left([0, \infty) ; H^{1} \times H_{*}^{2}\right) \cap H_{l o c}^{1}\left([0, \infty) ; L^{2}\right)$.

(iv) $\mathscr{P}(t)$ is decomposed as

$$
\mathscr{P}(t)=\mathscr{P}^{(0)}+\operatorname{div}^{\prime} \mathscr{P}^{(1)}(t)+\Delta^{\prime} \mathscr{P}^{(2)}(t) .
$$

Here,

$$
\mathscr{P}^{(0)} u=\mathscr{F}^{-1}\left\{\widehat{\chi}_{1}\left\langle\widehat{u}, u^{*(0)}\right\rangle\right\}=\mathscr{F}^{-1}\left\{\widehat{\chi}_{1}\left[Q_{0} \widehat{u}\right]\right\}
$$




$$
\begin{gathered}
\mathscr{P}^{(1)}(t) u=\mathscr{F}^{-1}\left\{\widehat{\chi}_{1}\left\langle\widehat{u}, u^{*(1)}(t)\right\rangle\right\}, \\
\mathscr{P}^{(2)}(t) u=\mathscr{F}^{-1}\left\{-\widehat{\chi}_{1}\left\langle\widehat{u}, u^{*(2)}\left(\xi^{\prime}, t\right)\right\rangle\right\} .
\end{gathered}
$$

$\mathscr{P}^{(p)}(t), p=0,1,2$, share the same properties given in (i) and (ii) for $\mathscr{P}(t)$.

(v) There holds

$$
\begin{gathered}
\left\|\partial_{x^{\prime}}^{k} e^{(t-s) \Lambda} \mathscr{P}(s) u\right\|_{L^{2}\left(\mathbb{R}^{n-1}\right)} \leq C(1+t-s)^{-\frac{n-1}{2}\left(\frac{1}{p}-\frac{1}{2}\right)-\frac{k}{2}}\|u\|_{p}, \\
\left\|\partial_{x^{\prime}}^{k} e^{(t-s) \Lambda} \mathscr{P}^{(q)}(s) u\right\|_{L^{2}\left(\mathbb{R}^{n-1}\right)} \leq C(1+t-s)^{-\frac{n-1}{2}\left(\frac{1}{p}-\frac{1}{2}\right)-\frac{k}{2}}\|u\|_{p}, \quad q=0,1,2,
\end{gathered}
$$

for $u \in L^{p}, 1 \leq p \leq 2$ and $k=0,1, \ldots$

Remark 4.17 Note that $\Pi^{(0)}(t)$ and $\mathscr{Q}^{(0)}(t) \mathscr{P}^{(0)}$ are not identical operators.

To close this section, we show improvements of regularity for $\phi$.

Proposition 4.18 Let $u={ }^{T}(\phi, w) \in Z^{m}(\tau)$ for $m \geq[n / 2]+1$ be a solution of (4.1). There holds

$$
\phi \in \bigcap_{j=1}^{\left[\frac{m+1}{2}\right]} C^{j}\left([0, \tau] ; H^{m+1-2 j}\right) .
$$

Proof. From definition of $Z^{m}(\tau)$ we have

$$
u \in \bigcap_{j=0}^{\left[\frac{m}{2}\right]} C^{j}\left([0, \tau] ; H^{m-2 j}\right) \text { and } \sup _{0 \leq z \leq \tau} \llbracket u(z) \rrbracket_{m}<\infty .
$$

We write (1.5) as

$$
\partial_{t} \phi=-v_{p}^{1} \partial_{x_{1}} \phi-\gamma^{2} \operatorname{div}\left(\rho_{p} w\right)-\operatorname{div}(\phi w) .
$$

Taking $\llbracket \cdot \rrbracket_{m-1}$-norm we obtain

$$
\llbracket \partial_{t} \phi \rrbracket_{m-1} \leq \llbracket v_{p}^{1} \partial_{x_{1}} \phi \rrbracket_{m-1}+\gamma^{2} \llbracket \rho_{p} w \rrbracket_{m}+\llbracket \phi w \rrbracket_{m} .
$$

Since $m \geq[n / 2]+1$ we get using Lemma 8.3 (ii) that

$$
\llbracket \partial_{t} \phi \rrbracket_{m-1} \leq C\left(\llbracket \phi \rrbracket_{m}\right)\left\{\llbracket v_{p}^{1} \rrbracket_{m} \llbracket \phi \rrbracket_{m}+\left\|\rho_{p}\right\|_{H^{m}} \llbracket w \rrbracket_{m}+\llbracket \phi \rrbracket_{m} \llbracket w \rrbracket_{m}\right\} .
$$

This concludes the proof.

\section{Decomposition of the solution}

In this section we decompose solution $u(t)$ of $(4.1)$ and we prove the a priori estimate in Proposition 3.7. We decompose $u(t)$ into several parts based on the spectral properties of $L(t)$. In this section we assume that $\nu \geq \nu_{2}$ and $\gamma^{2} /(\nu+\widetilde{\nu}) \geq \gamma_{2}^{2}$ unless further restricted.

Let us first introduce some notation and projection operators. Let $\hat{\chi}_{2}$ and $\widehat{\chi}_{3}$ be defined by

$$
\widehat{\chi}_{2}\left(\xi^{\prime}\right)=\mathbf{1}_{\left[r_{1}, 1\right)}\left(\left|\xi^{\prime}\right|\right) \text { and } \widehat{\chi}_{3}\left(\xi^{\prime}\right)=\mathbf{1}_{[1, \infty)}\left(\left|\xi^{\prime}\right|\right) .
$$

We then define $[f]_{j}, j=1,2, \infty$ by

$$
\begin{gathered}
{[f]_{j}=\mathscr{F}^{-1}\left(\widehat{\chi}_{j}[\widehat{f}]\right), \quad j=1,2,} \\
{[f]_{\infty}=[f]_{1}+[f]_{2}=\mathscr{F}^{-1}\left(\left(\widehat{\chi}_{1}+\widehat{\chi}_{2}\right)[\widehat{f}]\right) .}
\end{gathered}
$$

Next, we define $P_{\infty, j}, j=1,2,3$ as 


$$
\begin{gathered}
P_{\infty, 1}(t) u=\mathscr{F}^{-1}\left(\widehat{P}_{\infty, 1}(t) \widehat{u}\right), \quad \widehat{P}_{\infty, 1}(t) \widehat{u}=\widehat{\chi}_{1}\left(I-\widehat{\mathscr{Q}}_{\xi^{\prime}}(t) \widehat{\mathscr{P}}_{\xi^{\prime}}(t)\right) \widehat{u}, \\
P_{\infty, j} u=\mathscr{F}^{-1}\left(\widehat{P}_{\infty, j} \widehat{u}\right), \quad \widehat{P}_{\infty, j} \widehat{u}=\widehat{\chi}_{j} \widehat{u} \quad(j=2,3) .
\end{gathered}
$$

By setting

$$
\widetilde{P}_{\infty}(t)=I-\mathbb{P}(t), \quad P_{\infty}^{(0)}(t)=P_{\infty, 1}(t)+P_{\infty, 2},
$$

we get

$$
I=\mathbb{P}(t)+\widetilde{P}_{\infty}(t), \quad \widetilde{P}_{\infty}(t)=P_{\infty}^{(0)}(t)+P_{\infty, 3} .
$$

Using above operators we decompose solution $u(t)$ into

$$
u(t)=\mathbb{P}(t) u(t)+\widetilde{P}_{\infty}(t) u(t)
$$

with

$$
\begin{gathered}
\mathbb{P}(t) u(t)=\sigma_{1}(t) u^{(0)}(t)+u_{1}(t), \\
\widetilde{P}_{\infty}(t) u(t)=\sigma_{\infty}(t) u^{(0)}(t)+u_{\infty}(t),
\end{gathered}
$$

where

$$
\begin{gathered}
\sigma_{1}(t)=\mathscr{P}(t) u(t), \quad u_{1}(t)=\left(\mathscr{Q}(t)-\mathscr{Q}^{(0)}(t)\right) \mathscr{P}(t) u(t), \\
\sigma_{\infty}(t)=\left[Q_{0} P_{\infty}^{(0)}(t) u(t)\right]=\left[Q_{0} P_{\infty}^{(0)}(t) u(t)\right]_{\infty}, \quad u_{\infty}(t)=P_{\infty}(t) u(t) .
\end{gathered}
$$

By $P_{\infty}$ we denote the operator defined as

$$
P_{\infty}(t)=\left(I-\Pi^{(0)}(t)\right) P_{\infty}^{(0)}(t)+P_{\infty, 3}
$$

Remark 5.1 Notice that $\sigma_{1}(t), \sigma_{\infty}(t)$ and $u^{(0)}(t)$ are separate functions. Furthermore, notice that

$$
\left\|u_{1}(t)\right\|_{2} \leq C\left\|\partial_{x^{\prime}} \sigma_{1}(t)\right\|_{2}
$$

Next, we derive the equations for $\sigma_{1}, \sigma_{\infty}$ and $u_{\infty}$. We define $\mathscr{M}(t)$ by

$$
\mathscr{M}(t)=\widetilde{A}+\widetilde{B}(t),
$$

with

$$
\widetilde{A}=\left(\begin{array}{cc}
0 & 0 \\
0 & -\frac{\nu}{\rho_{p}} \Delta^{\prime} I_{n-1}-\frac{\widetilde{\nu}}{\rho_{p}} \nabla^{\prime} \operatorname{div} \\
0 & \left(-\frac{\widetilde{\nu}}{\rho_{p}} \partial_{x_{n}} \operatorname{div}^{\prime},-\frac{\nu}{\rho_{p}} \Delta^{\prime}\right)
\end{array}\right), \quad \widetilde{B}(t)=\left(\begin{array}{ccc}
v_{p}^{1}(t) \partial_{x_{1}} & \gamma^{2} \rho_{p} \operatorname{div}^{\prime} & 0 \\
\frac{P^{\prime}\left(\rho_{p}\right)}{\gamma^{2} \rho_{p}} \nabla^{\prime} & v_{p}^{1}(t) \partial_{x_{1}} I_{n-1} & 0 \\
0 & 0 & v_{p}^{1}(t) \partial_{x_{1}}
\end{array}\right)
$$

Proposition 5.2 Let $\tau>0$ and $u(t)$ be a solution of $(4.1)$ in $Z^{m}(\tau)$. Then there hold

$$
\begin{gathered}
\sigma_{k} \in \bigcap_{j=0}^{\left[\frac{m}{2}\right]} C^{j}\left([0, \tau] ; H^{l}\left(\mathbb{R}^{n-1}\right)\right),\left.\int_{0}^{\tau}\left\|D \sigma_{k}(z)\right\|\right|_{m} d z<\infty, k=1, \infty, l=0,1, \ldots, \\
u_{1} \in \bigcap_{j=0}^{\left[\frac{m}{2}\right]} C^{j}\left([0, \tau] ; H^{m+1-2 j}\right), \int_{0}^{\tau}\left\|\left|D w_{1}(z) \|\right|_{m} d z<\infty,\right. \\
u_{\infty} \in Z^{m}(\tau), \int_{0}^{\tau} \llbracket \partial_{t} \phi_{\infty}(z) \rrbracket_{m-1} d z<\infty .
\end{gathered}
$$

Moreover, $\sigma_{1}, \sigma_{\infty}$ and $u_{\infty}$ satisfy

$$
\sigma_{1}(t)=e^{(t-s) \Lambda} \mathscr{P}(s) u_{0}+\int_{s}^{t} e^{(t-z) \Lambda} \mathscr{P}(z) \boldsymbol{F}(z) d z,
$$




$$
\begin{gathered}
\partial_{t} \sigma_{\infty}+\left[Q_{0} \widetilde{B}\left(\sigma_{\infty} u^{(0)}+u_{\infty}\right)\right]_{\infty}=\left[Q_{0} P_{\infty}^{(0)} \boldsymbol{F}\right]_{\infty} \\
\partial_{t} u_{\infty}+L(t) u_{\infty}+\mathscr{M}(t)\left(\sigma_{\infty} u^{(0)}\right)-\left[Q_{0} \widetilde{B}\left(\sigma_{\infty} u^{(0)}+u_{\infty}\right)\right]_{\infty} u^{(0)}=P_{\infty} \boldsymbol{F} \\
\left.w_{\infty}\right|_{x_{n}=0,1}=0 \\
\left.\sigma_{\infty}\right|_{t=0}=\sigma_{\infty, 0},\left.\quad u_{\infty}\right|_{t=0}=u_{\infty, 0} .
\end{gathered}
$$

Here, $\sigma_{\infty, 0}=\left[Q_{0} P_{\infty}^{(0)}(0) u_{0}\right]_{\infty}, u_{\infty, 0}=P_{\infty} u_{0}$.

Proof. Since $u \in Z^{m}(\tau)$, the regularity assertions on $\sigma_{k}(k=1, \infty)$ and $u_{1}$ follow from properties of $\mathscr{P}(t)$, $\mathscr{Q}(t)$ and (4.15). As for $u_{\infty}$, we already know that $\mathbb{P} Z^{m}(\tau) \subset Z^{m}(\tau)$ and therefore $\widetilde{P}_{\infty} Z^{m}(\tau) \subset Z^{m}(\tau)$. Since it is straightforward to see that $P_{\infty, 3} Z^{m}(\tau) \subset Z^{m}(\tau)$ we have $P_{\infty}^{(0)} Z^{m}(\tau) \subset Z^{m}(\tau)$. Finally, from properties of $u^{(0)}(t)$ we obtain $\Pi^{(0)} P_{\infty}^{(0)} Z^{m}(\tau) \subset Z^{m}(\tau)$. Therefore, $u_{\infty} \in Z^{m}(\tau) . \int_{0}^{\tau} \llbracket \partial_{t} \phi_{\infty}(z) \rrbracket_{m-1} d z<\infty$ follows in analogous way using (4.15).

As for (5.1), it follows from (4.1) and (4.12) that

$$
\left(\partial_{t}-\Lambda\right) \sigma_{1}(t)=\mathscr{P}(t) \boldsymbol{F}(t) .
$$

The rest is standard.

As for (5.2) and (5.3), we first apply $\widetilde{P}_{\infty}(t)$ to $(4.1)$ to get

$$
\partial_{t}\left(\widetilde{P}_{\infty} u\right)+L(t) \widetilde{P}_{\infty} u=\widetilde{P}_{\infty} \boldsymbol{F} .
$$

Next, we apply $P_{\infty}^{(0)}(t)$ and $P_{\infty, 3}$ to (5.4) to obtain

$$
\begin{gathered}
\partial_{t}\left(P_{\infty}^{(0)} u\right)+L(t) P_{\infty}^{(0)} u=P_{\infty}^{(0)} \boldsymbol{F}, \\
\partial_{t}\left(P_{\infty, 3} u\right)+L(t) P_{\infty, 3} u=P_{\infty, 3} \boldsymbol{F} .
\end{gathered}
$$

Since $\left[Q_{0} L(t) v\right]=\left[Q_{0} \mathscr{M}(t) v\right]$ for $\left.\widetilde{Q} v\right|_{x_{2}=0,1}=0$ and $\left[Q_{0} \mathscr{M}(t) v\right]=\left[Q_{0} \widetilde{B}(t) v\right]$ for any $v$, we get by applying $\left[Q_{0} \cdot\right]$ to $(5.5)$

$$
\partial_{t} \sigma_{\infty}+\left[Q_{0} \widetilde{B}(t) P_{\infty}^{(0)} u\right]=\left[Q_{0} P_{\infty}^{(0)} \boldsymbol{F}\right]
$$

There holds

$$
\left[Q_{0} \widetilde{B}(t) P_{\infty}^{(0)} u\right]=\left[Q_{0} \widetilde{B}\left(\sigma_{\infty} u^{(0)}(t)+\left(I-\Pi^{(0)}(t)\right) P_{\infty}^{(0)} u\right)\right]_{\infty}=\left[Q_{0} \widetilde{B}\left(\sigma_{\infty} u^{(0)}+u_{\infty}\right)\right]_{\infty},
$$

and thus (5.2) follows from (5.7).

To obtain (5.3) we use the fact that $\partial_{t} \Pi^{(0)}(t)+L(t) \Pi^{(0)}(t)=\mathscr{M}(t) \Pi^{(0)}(t)$. Applying $I-\Pi^{(0)}(t)$ to (5.5) gives us

$\partial_{t}\left(\left(I-\Pi^{(0)}(t)\right) P_{\infty}^{(0)} u\right)+L(t)\left(I-\Pi^{(0)}(t)\right) P_{\infty}^{(0)} u+\mathscr{M}(t)\left(\sigma_{\infty} u^{(0)}(t)\right)-\Pi^{(0)}(t) \widetilde{B}(t)\left(P_{\infty}^{(0)} u\right)=\left(I-\Pi^{(0)}(t)\right) P_{\infty}^{(0)} \boldsymbol{F}$.

(5.3) now follows by adding (5.6) and (5.8). This completes the proof.

Let us state some properties of $\sigma_{1}, \sigma_{\infty}$ and $u_{\infty}$ parts.

Lemma 5.3 There hold the following inequalities.

$$
\left\|\partial_{x^{\prime}}^{k}\left[Q_{0} P_{\infty}^{(0)} u\right]_{\infty}\right\|_{2} \leq\left\|\left[Q_{0} P_{\infty}^{(0)} u\right]_{\infty}\right\|_{2}, \quad k=0,1, \ldots
$$

(ii)

$$
\left\|P_{\infty} u\right\|_{2} \leq C\left\|\partial_{x} P_{\infty} u\right\|_{2} \text { if }\left.\widetilde{Q} u\right|_{x_{n}=0,1}=0 .
$$


(iii) Let $\tau>0$ and $u(t)$ be a solution of $(4.1)$ in $Z^{m}(\tau)$. Then there hold

$$
\begin{gathered}
\left\|\partial_{x^{\prime}}^{k} \sigma_{1}\right\|_{2} \leq C\left\|\partial_{x^{\prime}} \sigma_{1}\right\|_{2},\left\|\partial_{x^{\prime}}^{k} \sigma_{\infty}\right\|_{2} \leq C\left\|\partial_{x^{\prime}} \sigma_{\infty}\right\|_{2}, k=1,2, \ldots, \\
\left\|\phi_{\infty}\right\|_{2} \leq C\left\|\partial_{x} \phi_{\infty}\right\|_{2} \\
\left\|w_{\infty}\right\|_{2} \leq C\left\|\partial_{x} w_{\infty}\right\|_{2} \\
\left\|\Lambda \sigma_{1}\right\|_{2} \leq C\left\|\partial_{x^{\prime}} \sigma_{1}\right\|_{2}
\end{gathered}
$$

Proof. Inequality (i) is obvious since $\operatorname{supp}\left(\widehat{\chi}_{1}+\widehat{\chi}_{2}\right) \subset\left\{\left|\xi^{\prime}\right| \leq 1\right\}$. As for (ii), since supp $\widehat{\chi}_{3} \subset\left\{\left|\xi^{\prime}\right| \geq 1\right\}$, we see that

$$
\left\|P_{\infty, 3} u\right\|_{2} \leq\left\|\partial_{x^{\prime}} P_{\infty, 3} u\right\|_{2}
$$

Since $\left.\widetilde{Q} u\right|_{x_{n}=0,1}=0$, we have $\left.\widetilde{Q} P_{\infty}^{(0)} u\right|_{x_{n}=0,1}=0$, and hence, $\left.\widetilde{Q}\left(I-\Pi^{(0)}(t)\right) P_{\infty}^{(0)} u\right|_{x_{n}=0,1}=0$. By the Poincaré ineguality we obtain

$$
\left\|\widetilde{Q}\left(I-\Pi^{(0)}(t)\right) P_{\infty}^{(0)} u\right\|_{2} \leq\left\|\partial_{x_{n}} \widetilde{Q}\left(I-\Pi^{(0)}(t)\right) P_{\infty}^{(0)} u\right\|_{2} .
$$

Furthermore, since $\left[Q_{0}\left(I-\Pi^{(0)}(t)\right) P_{\infty}^{(0)} u\right]=0$, we see from the Poincaré inequality that

$$
\left\|Q_{0}\left(I-\Pi^{(0)}(t)\right) P_{\infty}^{(0)} u\right\|_{2} \leq\left\|\partial_{x_{n}} Q_{0}\left(I-\Pi^{(0)}(t)\right) P_{\infty}^{(0)} u\right\|_{2} .
$$

It then follows that

$$
\left\|P_{\infty} u\right\|_{2} \leq C\left\{\left\|\partial_{x}\left(I-\Pi^{(0)}(t)\right) P_{\infty}^{(0)} u\right\|_{2}+\left\|\partial_{x} P_{\infty, 3} u\right\|_{2}\right\} \leq C\left\|\partial_{x} P_{\infty} u\right\|_{2} .
$$

Here, we used $\left(\partial_{x}\left(I-\Pi^{(0)}(t)\right) P_{\infty}^{(0)} u, \partial_{x} P_{\infty, 3} u\right)=0$, which follows from the fact $\widehat{\chi}_{1} \widehat{\chi}_{3}=\widehat{\chi}_{2} \widehat{\chi}_{3}=0$ and the Plancherel theorem.

As for (iii), it follows from the proof of (i) and (ii).

We prove the a priori estimate in Proposition 3.7 by estimating the following quantities.

Let $u(t)$ be solution of $(4.1)$ in $Z^{m}(\tau)$ and let $u(t)$ be decomposed as above, i.e.,

$$
u(t)=\sigma_{1}(t) u^{(0)}(t)+u_{1}(t)+\sigma_{\infty}(t) u^{(0)}(t)+u_{\infty}(t) .
$$

We define $M(t) \geq 0$ by

$$
M(t)^{2}=M_{1}(t)^{2}+\sup _{0 \leq z \leq t}(1+z)^{\frac{n+1}{2}} E_{\infty}(z), \quad t \in[0, \tau] .
$$

Here, $M_{1}(t)$ and $E_{\infty}(t)$ are defined as

$$
M_{1}(t)=\sup _{0 \leq z \leq t}(1+z)^{\frac{n-1}{4}}\left\|\sigma_{1}(z)\right\|_{2}+\sup _{0 \leq z \leq t}(1+z)^{\frac{n+1}{4}}\left\{\left\|\partial_{x^{\prime}} \sigma_{1}(z)\right\|_{2}+\sum_{j=1}^{\left[\frac{m}{2}\right]}\left\|\partial_{z}^{j} \sigma_{1}(z)\right\|_{2}\right\},
$$

and

$$
E_{\infty}(t)=\llbracket u_{\infty}(t) \rrbracket_{m}^{2}+\llbracket \sigma_{\infty}(t) \rrbracket_{m}^{2} .
$$

Finally, we introduce quantity $D_{\infty}(t)$ for $u_{\infty}(t)={ }^{T}\left(\phi_{\infty}(t), w_{\infty}(t)\right)$ :

$$
D_{\infty}(t)=\llbracket \partial_{x} \phi_{\infty}(t) \rrbracket_{m-1}^{2}+\llbracket \partial_{t} \phi_{\infty} \rrbracket_{m-1}^{2}+\left.\left\|\left|D w_{\infty}(t)\left\|\left.\right|_{m} ^{2}+\right\|\right| D \sigma_{\infty}(t)\right\|\right|_{m} ^{2} .
$$


Remark 5.4 From properties of $\mathscr{Q}^{(p)}(t), p=1,2$, we see that

$$
\left\|\partial_{x^{\prime}}^{k} \partial_{x_{n}}^{l} \partial_{t}^{j} u_{1}(t)\right\|_{2} \leq C\left\|\mid D \sigma_{1}(t)\right\| \|_{m}, 0 \leq 2 j+l \leq m+1, k=0,1, \ldots,
$$

and there holds

$$
\sup _{0 \leq z \leq t}(1+z)^{\frac{n+1}{4}}\left\{\llbracket u_{1}(z) \rrbracket_{m}+\llbracket \partial_{x} u_{1}(z) \rrbracket_{m}\right\} \leq C M_{1}(t) .
$$

Therefore, we do not need special estimates for $u_{1}(t)$.

We show the following estimates for $M_{1}(t)$ and $E_{\infty}(t)$.

Proposition 5.5 There exist positive constants $\nu_{0}$ and $\gamma_{0}$ such that if $\nu \geq \nu_{0}$ and $\gamma^{2} /(\nu+\widetilde{\nu}) \geq \gamma_{0}^{2}$, then the following assertions hold true.

There exists $\varepsilon_{2}>0$ such that if solution $u(t)$ of $(4.1)$ in $Z^{m}(\tau)$ satisfies $\sup _{0 \leq z \leq t} \llbracket u(z) \rrbracket_{m} \leq \varepsilon_{2}$ and $M(t) \leq 1$ for all $t \in[0, \tau]$, then the following estimates hold uniformly for $t \in[0, \tau]$ with $C>0$ independent of $\tau$.

$$
M_{1}(t) \leq C\left\{\left\|u_{0}\right\|_{1}+M(t)^{2}\right\}
$$

$$
E_{\infty}(t)+\int_{0}^{t} e^{-a(t-z)} D_{\infty}(z) d z \leq C\left\{e^{-a t} E_{\infty}(0)+(1+t)^{-\frac{n+1}{2}} M(t)^{4}+\int_{0}^{t} e^{-a(t-z)} \widetilde{R}(z) d z\right\}
$$

Here, $a=a(\nu, \widetilde{\nu}, \gamma)$ is a positive constant; and $\widetilde{R}(t)$ is quantity that satisfies

$$
\widetilde{R}(t) \leq C\left\{(1+t)^{-\frac{n+1}{2}} M(t)^{3}+M(t) D_{\infty}(t)\right\}
$$

whenever $\sup _{0 \leq z \leq t} \llbracket u(z) \rrbracket_{m} \leq \varepsilon_{2}$ and $M(t) \leq 1$.

The proof of Proposition 5.5 is given in Sections 6-8. We prove (5.9), (5.10) and (5.11) in Sections 6,7 and 8 , respectively.

Assuming that Proposition 5.5 holds true, we can show the following estimate.

Proposition 5.6 If $\nu \geq \nu_{0}$ and $\gamma^{2} /(\nu+\widetilde{\nu}) \geq \gamma_{0}^{2}$, then the following assertion holds true. There exists number $\varepsilon_{3}>0$ such that if solution $u(t)$ of (4.1) in $Z^{m}(\tau)$ satisfies $\left\|u_{0}\right\|_{H^{m} \cap L^{1}} \leq \varepsilon_{3}$, then there holds the estimate

$$
M(t) \leq C\left\|u_{0}\right\|_{H^{m} \cap L^{1}}
$$

for a constant $C>0$ independent of $\tau$.

As an immediate consequence of (5.12) we see that the a priory estimate in Proposition 3.7 holds true. Moreover, (5.12) provides us with the following decay estimates:

$$
\begin{gathered}
\llbracket u(t) \rrbracket_{m} \leq C(1+t)^{-\frac{n-1}{4}}\left\|u_{0}\right\|_{H^{m} \cap L^{1}}, \\
\left\|\partial_{x^{\prime}}^{k} u(t)\right\|_{2} \leq C(1+t)^{-\frac{n-1}{4}-\frac{k}{2}}\left\|u_{0}\right\|_{H^{m} \cap L^{1}}, k=0,1,
\end{gathered}
$$

and

$$
\left\|u(t)-\sigma_{1}(t) u^{(0)}(t)\right\|_{2} \leq C(1+t)^{-\frac{n+1}{4}}\left\|u_{0}\right\|_{H^{m} \cap L^{1}}
$$

for $t \in[0, \tau]$. This proves (3.6) and (3.8).

Proof of Proposition 5.6 If $\sup _{0 \leq z \leq t} \llbracket u(z) \rrbracket_{m} \leq \varepsilon_{2}$ and $M(t) \leq 1$, then we see from (5.10) and (5.11) that

$$
\begin{aligned}
E_{\infty}(t) & +\int_{0}^{t} e^{-a(t-z)} D_{\infty}(z) d z \leq C\left\{e^{-a t} E_{\infty}(0)+(1+t)^{-\frac{n+1}{2}} M(t)^{4}\right. \\
& \left.+\int_{0}^{t} e^{-a(t-z)}\left\{(1+z)^{-\frac{n+1}{2}} M(z)^{3}+M(z) D_{\infty}(z)\right\} d z\right\}
\end{aligned}
$$




$$
\leq C\left\{e^{-a t} E_{\infty}(0)+(1+t)^{-\frac{n+1}{2}} M(t)^{3}+M(t) \int_{0}^{t} e^{-a(t-z)} D_{\infty}(z) d z\right\} .
$$

Therefore, using continuity of $E_{\infty}(t)$ and compatibility conditions we obtain

$$
(1+t)^{\frac{n+1}{2}} E_{\infty}(t)+\mathscr{D}(t)+\leq C\left\{\left\|u_{0}\right\|_{H^{m}}^{2}+M(t)^{3}+M(t) \mathscr{D}(t)\right\},
$$

with

$$
\mathscr{D}(t)=(1+t)^{\frac{n+1}{2}} \int_{0}^{t} e^{-a(t-z)} D_{\infty}(z) d z .
$$

It follows from (5.9) and (5.14) that

$$
M(t)^{2}+\sup _{0 \leq z \leq t} \mathscr{D}(z) \leq C_{1}\left\{\left\|u_{0}\right\|_{H^{m} \cap L^{1}}^{2}+M(t)^{3}+M(t) \sup _{0 \leq z \leq t} \mathscr{D}(t)\right\},
$$

whenever $\sup _{0 \leq z \leq t} \llbracket u(z) \rrbracket_{m} \leq \varepsilon_{2}$ and $M(t) \leq 1$.

In the same way as in [7, Proof of Proposition 5.4] using (5.15) one can show that there exists $\varepsilon_{3}>0$ such that if $\left\|u_{0}\right\|_{H^{m} \cap L^{1}}<\varepsilon_{3}$, then

$$
M(t)<2 C_{2}\left\|u_{0}\right\|_{H^{m} \cap L^{1}},
$$

for all $t \in[0, \tau]$ with $C_{2}>0$ independent of $\tau$. This concludes the proof.

\section{Estimates on $\sigma_{1}(t)$}

In this section we estimate the $\mathbb{P}(t)$-part of $u(t)$. Since

$$
\mathbb{P}(t) u(t)=\sigma_{1}(t) u^{(0)}(t)+u_{1}(t),
$$

where $\sigma_{1}(t)=\mathscr{P}(t) u(t)$ and $u_{1}(t)=\left(\mathscr{Q}(t)-\mathscr{Q}^{(0)}(t)\right) \mathscr{P}(t) u(t)$, it is enough to obtain estimates for $\sigma_{1}$ (see Remark 5.4). In this section we assume that $\nu \geq \nu_{2}$ and $\gamma^{2} /(\nu+\widetilde{\nu}) \geq \gamma_{2}^{2}$.

Let us first make an observation. Regarding the spectral properties of linearized operator, we expect $\sigma_{1}(t)$ to be the most slowly decaying part of $u(t)$. Therefore, the most slowly decaying part of the nonlinearity $\boldsymbol{F}(t, u(t))$ would be given by the terms containing only $\sigma_{1}(t)^{2}$. There are two such terms in $\boldsymbol{F}(t, u(t))$,

$$
\frac{\nu \phi}{\gamma^{2} \rho_{p}^{2}}\left(-\partial_{x_{n}}^{2} w^{1}+\frac{\partial_{x_{n}}^{2} v_{p}^{1}}{\gamma^{2} \rho_{p}} \phi\right) e_{1} \quad \text { and } \quad-\frac{1}{2 \gamma^{4} \rho_{p}} \partial_{x_{n}}\left(P^{\prime \prime}\left(\rho_{p}\right) \phi^{2}\right) e_{n}
$$

Since $w^{(0), 1}$ satisfies (4.7), we can define $\sigma_{1}^{2} \boldsymbol{F}_{1}$ with $\boldsymbol{F}_{1}=\boldsymbol{F}_{1}\left(x_{n}, t\right)$ as

$$
\boldsymbol{F}_{1}={ }^{T}\left(0, \frac{\phi^{(0)}\left(x_{n}\right)}{\gamma^{2} \rho_{p}} \partial_{t} w^{(0), 1}\left(x_{n}, t\right), \frac{1}{2 \gamma^{4} \rho_{p}\left(x_{n}\right)} \partial_{x_{n}}\left(P^{\prime \prime}\left(\rho_{p}\left(x_{2}\right)\right)\left\{\phi^{(0)}\left(x_{n}\right)\right\}^{2}\right)\right) \text {. }
$$

We thus write

$$
\boldsymbol{F}=\sigma_{1}^{2} \boldsymbol{F}_{1}+\boldsymbol{F}_{2}
$$

where $\boldsymbol{F}_{2}=\boldsymbol{F}-\sigma_{1}^{2} \boldsymbol{F}_{1}$ contains terms involving $u_{\infty}$, its derivatives and terms of order $O\left(\sigma_{1} \partial_{x^{\prime}} \sigma_{1}\right)$ like $\sigma_{1} u_{1}$, and $O\left(\sigma_{1}^{3}\right)$, but not just $O\left(\sigma_{1}^{2}\right)$. In particular, we have that $Q_{0} \boldsymbol{F}=Q_{0} \boldsymbol{F}_{2}$.

First we introduce two lemmas.

Lemma 6.1 There hold the following relations.

$$
\left[Q_{0} \boldsymbol{F}\right]=-\operatorname{div}^{\prime}\left[\phi w^{\prime}\right]
$$

(ii)

$$
\mathscr{P}(t) \boldsymbol{F}(t)=-\operatorname{div}^{\prime}\left[\phi(t) w^{\prime}(t)\right]_{1}+\operatorname{div}^{\prime} \mathscr{P}^{(1)}(t) \boldsymbol{F}(t)+\Delta^{\prime} \mathscr{P}^{(2)}(t) \boldsymbol{F}(t)
$$


Proof. Since $\left.w\right|_{x_{n}=0,1}=0$, by integration by parts, we have

$$
\left[Q_{0} \boldsymbol{F}\right]=-\operatorname{div}^{\prime}\left[\phi w^{\prime}\right] \text {. }
$$

This shows (i). As for (ii), it is straightforward from definition of $\mathscr{P}^{(0)}$ and (i).

Remark 6.2 Let $\varepsilon_{5}>0$ be number such that

$$
C_{s} \varepsilon_{5} \leq \frac{\gamma^{2} \rho_{1}}{4}
$$

Here, $C_{S}>0$ comes from Sobolev inequality (3.10). Then whenever $\llbracket u(t) \rrbracket_{m} \leq \varepsilon_{5}$, we have

$$
\|\phi(t)\|_{\infty} \leq C_{S} \llbracket u(t) \rrbracket_{m} \leq C_{S} \varepsilon_{5} \leq \frac{\gamma^{2} \rho_{1}}{4}
$$

and hence,

$$
\rho(x, t)=\rho_{p}\left(x_{2}\right)+\gamma^{-2} \phi(x, t) \geq \rho_{1}-\gamma^{-2}\|\phi(t)\|_{\infty} \geq \frac{3 \rho_{1}}{4}>0 .
$$

Therefore, we see that $\widetilde{Q} \boldsymbol{F}(t)$ is smooth whenever $\llbracket u(t) \rrbracket_{m} \leq \varepsilon_{5}$.

Using inequality $\left\|\sigma_{1}\right\|_{\infty} \leq C\left\|\sigma_{1}\right\|_{2}^{1 / 2}\left\|\partial_{x^{\prime}} \sigma_{1}\right\|_{2}^{1 / 2}$ (see Lemmas 8.2 (iii) and 5.3 (iii)), it is not difficult to verify the following estimates on nonlinearities. We omit the proof.

Lemma 6.3 Let solution $u(t)$ of (4.1) in $Z^{m}(\tau)$ satisfies $\sup _{0 \leq z \leq t} \llbracket u(z) \rrbracket_{m} \leq \varepsilon_{5}$ and $M(t) \leq 1$ for $t \in[0, \tau]$, then there hold the following estimates for $t \in[0, \tau]$ with $C>0$ independent of $\tau$.

$$
\left\|\partial_{x^{\prime}}\left(\sigma_{1}^{2}(t)\right)\right\|_{1} \leq C(1+t)^{-\frac{n}{2}} M(t)^{2}
$$

(ii)

$$
\left\|\operatorname{div}^{\prime}\left[\phi w^{\prime}\right](t)\right\|_{1} \leq C(1+t)^{-\frac{n}{2}} M(t)^{2}
$$

(iii)

(iv)

$$
\left\|\left[\phi w^{\prime}\right](t)\right\|_{1} \leq C(1+t)^{-\frac{n-1}{2}} M(t)^{2}
$$

$$
\|\boldsymbol{F}(t)\|_{1} \leq C(1+t)^{-\frac{n-1}{2}} M(t)^{2},
$$

(v)

$$
\left\|\boldsymbol{F}_{2}(t)\right\|_{1} \leq C(1+t)^{-\frac{n}{2}} M(t)^{2},
$$

(vi)

$$
\|\boldsymbol{F}(t)\|_{2} \leq C(1+t)^{-\frac{2 n-1}{4}} M(t)^{2},
$$

(vii)

$$
\left\|\partial_{x^{\prime}}\left(\sigma_{1}^{2}(t)\right)\right\|_{2} \leq C(1+t)^{-\frac{2 n+1}{4}} M(t)^{2} .
$$

Finally, we prove (5.9).

Proposition 6.4 There exists number $\varepsilon_{4}>0$ such that if a solution $u(t)$ of $(4.1)$ in $Z^{m}(\tau)$ satisfies $\sup _{0 \leq z \leq t} \llbracket u(z) \rrbracket_{m} \leq \varepsilon_{4}$ and $M(t) \leq 1$ for all $t \in[0, \tau]$, then the estimate

$$
M_{1}(t) \leq C\left\{\left\|u_{0}\right\|_{1}+M(t)^{2}\right\}
$$

holds uniformly for $t \in[0, \tau]$ with $C>0$ independent of $\tau$. 
Proof. We write (5.1) for $s=0$ as

$$
\sigma_{1}(t)=e^{t \Lambda} \mathscr{P}(0) u_{0}+I(t)
$$

where

$$
I(t)=\int_{0}^{t} e^{(t-z) \Lambda} \mathscr{P}(z) F(z) d z
$$

(4.13) yields

$$
\left\|\partial_{x^{\prime}}^{k} e^{t \Lambda} \mathscr{P}(0) u_{0}\right\|_{2} \leq C(1+t)^{-\frac{n-1}{4}-\frac{k}{2}}\left\|u_{0}\right\|_{1}
$$

for $k=0,1$. Next, we estimate $I(t)$ which we write it as

$$
I(t)=I_{1}(t)+I_{2}(t)
$$

where

$$
\begin{aligned}
& I_{1}(t)=\int_{0}^{\frac{t}{2}} e^{(t-z) \Lambda} \mathscr{P}(z) F(z) d z, \\
& I_{2}(t)=\int_{\frac{t}{2}}^{t} e^{(t-z) \Lambda} \mathscr{P}(z) F(z) d z .
\end{aligned}
$$

By Lemma 6.1 (ii), we have

$$
e^{(t-z) \Lambda} \mathscr{P}(z) \boldsymbol{F}(z)=\operatorname{div}^{\prime} e^{(t-z) \Lambda}\left\{-\left[\phi w^{\prime}\right]_{1}+\mathscr{P}^{(1)} \boldsymbol{F}+\nabla^{\prime} \mathscr{P}^{(2)} \boldsymbol{F}\right\}(z) .
$$

It then follows from (4.14) and Lemma 6.3 that

$$
\begin{gathered}
\left\|\partial_{x^{\prime}}^{k} I_{1}(t)\right\|_{2} \leq C \int_{0}^{\frac{t}{2}}(1+t-z)^{-\frac{n+1}{4}-\frac{k}{2}}\left\{\left\|\left[\phi w^{\prime}\right](z)\right\|_{1}+\|\boldsymbol{F}(z)\|_{1}\right\} d z \\
\leq C M(t)^{2} \int_{0}^{\frac{t}{2}}(1+t-z)^{-\frac{n+1}{4}-\frac{k}{2}}(1+z)^{-\frac{n-1}{2}} d z \leq C(1+t)^{-\frac{n-1}{4}-\frac{k}{2}} M(t)^{2},
\end{gathered}
$$

for $k=0,1$.

As for $I_{2}(t)$, using (6.1) and Lemma 6.1 (ii) we write $\mathscr{P}(z) \boldsymbol{F}(z)$ as

$$
\mathscr{P} \boldsymbol{F}=-\operatorname{div}^{\prime}\left[\phi w^{\prime}\right]_{1}+\left(\mathscr{P}^{(1)}+\nabla^{\prime} \mathscr{P}^{(2)}\right) \cdot \nabla^{\prime}\left(\sigma_{1}\right)^{2} \boldsymbol{F}_{1}+\left(\operatorname{div}^{\prime} \mathscr{P}^{(1)}+\Delta^{\prime} \mathscr{P}^{(2)}\right) \boldsymbol{F}_{2} .
$$

It then follows from (4.14) and Lemma 6.3 that

$$
\begin{gathered}
\left\|\partial_{x^{\prime}}^{k} I_{2}(t)\right\|_{2} \leq C \int_{\frac{t}{2}}^{t}(1+t-z)^{-\frac{n-1}{4}-\frac{k}{2}}\left\{\left\|\operatorname{div}^{\prime}\left[\phi w^{\prime}\right](z)\right\|_{1}+\left\|\nabla^{\prime}\left(\sigma_{1}(z)\right)^{2}\right\|_{1}+\left\|\boldsymbol{F}_{2}(z)\right\|_{1}\right\} d z \\
\leq C M(t)^{2} \int_{\frac{t}{2}}^{t}(1+t-z)^{-\frac{n-1}{4}-\frac{k}{2}}(1+z)^{-\frac{n}{2}} d z \leq C(1+t)^{-\frac{n-1}{4}-\frac{k}{2}} M(t)^{2},
\end{gathered}
$$

for $k=0,1$. We thus obtain

$$
\sum_{k=0}^{1}(1+t)^{\frac{n-1}{4}+\frac{k}{2}}\left\|\partial_{x^{\prime}}^{k} \sigma_{1}(t)\right\|_{2} \leq C\left\{\left\|u_{0}\right\|_{1}+M(t)^{2}\right\} .
$$

It remains to estimate time derivatives. From (5.1) we see that

$$
\partial_{t} \sigma_{1}(t)=\Lambda \sigma_{1}(t)+\mathscr{P}(t) \boldsymbol{F}(t) .
$$

It then follows from Lemma 6.3 (vi) and previous result that

$$
\left\|\partial_{t} \sigma_{1}(t)\right\|_{2} \leq C\left\{\left\|\partial_{x^{\prime}} \sigma_{1}(t)\right\|_{2}+\|\mathscr{P}(t) \boldsymbol{F}(t)\|_{2}\right\} \leq C(1+t)^{-\frac{n+1}{4}}\left\{\left\|u_{0}\right\|_{1}+M(t)^{2}\right\} .
$$

Concerning $\left\|\partial_{t}^{j+1} \sigma_{1}(t)\right\|_{2}$ for $j=1, \ldots,\left[\frac{m}{2}\right]-1$, we obtain from (6.3)

$$
\left\|\partial_{t}^{j+1} \sigma_{1}(t)\right\|_{2} \leq C\left\{\left\|\partial_{t}^{j} \sigma_{1}(t)\right\|_{2}+\left\|\partial_{t}^{j}(\mathscr{P}(t) \boldsymbol{F}(t))\right\|_{2}\right\} .
$$


Since

$$
\left\|\partial_{t}^{j} \boldsymbol{F}(t)\right\|_{2} \leq C(1+t)^{-\frac{n+1}{4}} M(t)^{2}
$$

for $0 \leq 2 j \leq m-2$ as we see in Propositions 8.5 (i)-(iii) and 8.6 (i), we find by induction on $j$, that estimate

$$
\left\|\partial_{t}^{j+1} \sigma_{1}(t)\right\|_{2} \leq C_{j}(1+t)^{-\frac{n+1}{4}}\left\{\left\|u_{0}\right\|_{1}+M(t)^{2}\right\}
$$

holds for $j=0,1, \ldots,\left[\frac{m}{2}\right]-1$.

The desired result now follows from (6.2) and (6.4). This completes the proof.

\section{Estimates on $\widetilde{P}_{\infty} u(t)$}

In this section we prove estimate (5.10) for $\sigma_{\infty}$ and $u_{\infty}$ by a variant of Matsumura-Nishida energy method as in the case of stationary parallel flow $([7])$. Since coefficients of the linearized operator depend on time some extra terms arise in contrast to [7]. We omit the proofs that can be obtained as modification of those in [7]. In this section we assume that $\nu \geq \nu_{2}$ and $\gamma^{2} /(\nu+\widetilde{\nu}) \geq \gamma_{2}^{2}$ unless further restricted.

First, let us show the following inequality.

Proposition 7.1 There exists $\nu_{0} \geq \nu_{2}$ and $\gamma_{0} \geq \gamma_{2}$ such that if $\nu \geq \nu_{0}$ and $\gamma^{2} /(\nu+\widetilde{\nu}) \geq \gamma_{0}^{2}$ the solution $u(t)$ of $(4.1)$ in $Z^{m}(\tau)$ satisfies

$$
\frac{d}{d t} \widetilde{E}(t)+2 D(t) \leq \widetilde{R}(t)
$$

Here, $\widetilde{E}(t), D(t)$ and $\widetilde{R}(t)$ are quantities such that

(i) $\widetilde{E}(t)+\llbracket \partial_{x_{n}}^{2} w_{\infty}(t) \rrbracket_{m-2}^{2}$ is equivalent to $E_{\infty}(t)$,

(ii) $D(t)$ is equivalent to $D_{\infty}(t)$,

(iii) $\widetilde{R}(t)$ satisfies estimate (5.11).

We introduce some quantities. Let $E^{(0)}\left[\widetilde{P}_{\infty} u\right]$ and $D^{(0)}[w]$ be defined by

$$
E^{(0)}\left[\widetilde{P}_{\infty} u\right]=\frac{\alpha_{0}}{\gamma^{2}}\left\|\sigma_{\infty}\right\|_{2}^{2}+\frac{1}{\gamma^{2}}\left\|\sqrt{\frac{P^{\prime}\left(\rho_{p}\right)}{\gamma^{2} \rho_{p}}} \phi_{\infty}\right\|_{2}^{2}+\left\|\sqrt{\rho_{p}} w_{\infty}\right\|_{2}^{2},
$$

for $\widetilde{P}_{\infty}(t)=\sigma_{\infty} u^{(0)}+u_{\infty}$ with $u_{\infty}={ }^{T}\left(\phi_{\infty}, w_{\infty}\right)$; and

$$
D^{(0)}\left[w_{\infty}\right]=\nu\left\|\nabla w_{\infty}\right\|_{2}^{2}+\widetilde{\nu}\left\|\operatorname{div} w_{\infty}\right\|_{2}^{2}
$$

Note that,

$$
\langle A u(t), u(t)\rangle_{\Omega}=D^{(0)}[w(t)]
$$

for $u={ }^{T}(\phi, w) \in Z^{m}(\tau)$, and

$$
\langle B(t) u(t), v(t)\rangle_{\Omega}=-\langle u(t), B(t) v(t)\rangle_{\Omega},
$$

for $u, v \in Z^{m}(\tau),\left.\widetilde{Q} u\right|_{x_{n}=0,1}=\left.\widetilde{Q} v\right|_{x_{n}=0,1}=0$. In particular,

$$
\langle B(t) u(t), u(t)\rangle_{\Omega}=0
$$

for $u \in Z^{m}(\tau),\left.\widetilde{Q} u\right|_{x_{n}=0,1}=0$.

We denote the tangential derivatives $\partial_{t}^{j} \partial_{x^{\prime}}^{k}$ by $T_{j, k}$ :

$$
T_{j, k} u=\partial_{t}^{j} \partial_{x^{\prime}}^{k} u \text {. }
$$

In this section we often use $\left\|w^{(0), 1}(t)\right\|_{C^{m+1}(\Omega)}=O\left(\frac{1}{\gamma^{2}}\right)$ in calculations (see Lemma 4.9). It is straightforward to see that following lemma holds true.

Lemma 7.2 There hold the following assertions. 
(i)

$$
\begin{gathered}
\left\|T_{j, k+1} \sigma_{\infty}\right\|_{2}^{2} \leq\left\|T_{j, 1} \sigma_{\infty}\right\|_{2}^{2}, k \geq 0,2 j \leq m, \\
\left\|T_{j, k} \phi_{\infty}\right\|_{2}^{2} \leq C\left\|\partial_{x} T_{j, k} \phi_{\infty}\right\|_{2}^{2}, 2 j+k \leq m-1, \\
\left\|T_{j, k} w_{\infty}\right\|_{2}^{2} \leq C\left\|\partial_{x} T_{j, k} w_{\infty}\right\|_{2}^{2}, 2 j+k \leq m-1 .
\end{gathered}
$$

(ii)

$$
\left\|\left[Q_{0} \widetilde{B}\left(\sigma_{\infty} u^{(0)}+u_{\infty}\right)\right]_{\infty}\right\|_{2}^{2} \leq C\left(\left\|\partial_{x^{\prime}} \sigma_{\infty}\right\|_{2}^{2}+\left\|\partial_{x^{\prime}} \phi_{\infty}\right\|_{2}^{2}+\gamma^{4}\left\|\partial_{x^{\prime}} w_{\infty}\right\|_{2}^{2}\right) .
$$

(iii) If $\left.w_{\infty}^{2}\right|_{x_{n}=0,1}=0$, then $\left[Q_{0} \widetilde{B} u_{\infty}\right]_{\infty}=\left[Q_{0} B u_{\infty}\right]_{\infty}=\left[v_{p}^{1} \partial_{x_{1}} \phi_{\infty}+\gamma^{2} \operatorname{div}\left(\rho_{p} w_{\infty}\right)\right]_{\infty}$.

(iv) If $\left.w_{\infty}^{2}\right|_{x_{n}=0,1}=0$ and $2 j+k \leq m$, then

$$
\begin{gathered}
\left\|\partial_{x^{\prime}}^{k} \partial_{t}^{j}\left[Q_{0} \widetilde{B}\left(\sigma_{\infty} u^{(0)}+u_{\infty}\right)\right]_{\infty}\right\|_{2}^{2} \\
\left.\leq C \sum_{i=0}^{j}\left(\left\|\partial_{x^{\prime}}^{p} \partial_{t}^{i} \sigma_{\infty}\right\|_{2}^{2}+\left\|\partial_{x^{\prime}}^{q} \partial_{t}^{i} \phi_{\infty}\right\|_{2}^{2}\right)+\gamma^{4}\left\|\operatorname{div}\left(\partial_{x^{\prime}}^{r} \partial_{t}^{j} w_{\infty}\right)\right\|_{2}^{2}+\gamma^{4}\left|\partial_{x_{n}} \rho_{p}\right|_{\infty}^{2}\left\|\partial_{x^{\prime}}^{s} \partial_{t}^{j} w_{\infty}\right\|_{2}^{2}\right),
\end{gathered}
$$

for $0 \leq p, q \leq k+1,0 \leq r, s \leq k$.

We begin with $L^{2}$-energy estimates for tangential derivatives. We set

$$
\begin{gathered}
\sigma_{*}=\sigma_{1}+\sigma_{\infty}, \quad \phi_{*}=\phi_{1}+\phi_{\infty}, \quad w_{*}=w_{1}+w_{\infty}, \\
u_{*}={ }^{T}\left(\phi_{*}, w_{*}\right)=u_{1}+u_{\infty} .
\end{gathered}
$$

We write $\widetilde{Q} \boldsymbol{F}={ }^{T}(0, \boldsymbol{f})$ in the form

$$
\widetilde{Q} \boldsymbol{F}=\widetilde{\boldsymbol{F}}_{0}+\widetilde{\boldsymbol{F}}_{1}+\widetilde{\boldsymbol{F}}_{2}+\widetilde{\boldsymbol{F}}_{3} .
$$

Here, $\widetilde{\boldsymbol{F}}_{l}={ }^{T}\left(0, \boldsymbol{f}_{l}\right), l=0,1,2,3$, with

$$
\begin{gathered}
\boldsymbol{f}_{0}=-w \cdot \nabla w-f_{1}\left(\rho_{p}, \phi\right) \Delta^{\prime} \sigma_{*} w^{(0), 1} \boldsymbol{e}_{1}-f_{2}\left(\rho_{p}, \phi\right) \nabla\left(\partial_{x_{1}} \sigma_{*} w^{(0), 1}\right) \\
+\boldsymbol{f}_{01}\left(x_{n}, t, \phi\right) \phi \sigma_{*}+\boldsymbol{f}_{02}\left(x_{n}, \phi\right) \phi \nabla^{\prime} \sigma_{*}+\boldsymbol{f}_{03}\left(x_{n}, t, \phi\right) \phi \phi_{*}, \\
\boldsymbol{f}_{1}=-f_{1}\left(\rho_{p}, \phi\right) \Delta w_{*}=-\operatorname{div}\left(f_{1}\left(\rho_{p}, \phi\right) \nabla w_{*}\right)+{ }^{T}\left(\nabla w_{*}\right) \nabla\left(f_{1}\left(\rho_{p}, \phi\right)\right), \\
\boldsymbol{f}_{2}=-f_{2}\left(\rho_{p}, \phi\right) \nabla \operatorname{div} w_{*}=-\nabla\left(f_{2}\left(\rho_{p}, \phi\right) \operatorname{div} w_{*}\right)+\left(\operatorname{div} w_{*}\right) \nabla\left(f_{2}\left(\rho_{p}, \phi\right)\right), \\
\boldsymbol{f}_{3}=-f_{3}\left(x_{n}, \phi\right) \phi \nabla \phi_{*} .
\end{gathered}
$$

Here, $\nabla w_{*}$ denotes the $n \times n$ matrix $\left(\partial_{x_{i}} w_{*}^{j}\right) ; f_{1}=\frac{\nu \phi}{\rho_{p}\left(\gamma^{2} \rho_{p}+\phi\right)} ; f_{2}=\frac{\widetilde{\nu} \phi}{\rho_{p}\left(\gamma^{2} \rho_{p}+\phi\right)} ;$ and $\boldsymbol{f}_{0 l}\left(x_{n}, t, \phi\right), l=1,2,3$ and $f_{3}\left(x_{n}, \phi\right)$ are smooth functions of $x_{n}, t$ and $\phi$.

Proposition 7.3 There exists $\nu_{3}>\nu_{2}$ such that for $\nu \geq \nu_{3}$ the following estimate holds for $0 \leq 2 j+k \leq m$ :

$$
\begin{gathered}
\frac{1}{2} \frac{d}{d t} E^{(0)}\left[T_{j, k} \widetilde{P}_{\infty} u\right]+\frac{1}{2} D^{(0)}\left[T_{j, k} w_{\infty}\right] \\
\leq R_{j, k}^{(1)}+C\left\{\left(\frac{\nu}{\gamma^{4}}+\frac{1}{\gamma^{4} \nu}+\frac{1}{\gamma^{2}}\right) \sum_{i=0}^{j}\left\|T_{i, k} \phi_{\infty}\right\|_{2}^{2}+\left(\frac{\nu+\widetilde{\nu}}{\gamma^{4}}+\frac{1}{\nu \gamma^{4}}+\frac{1}{\gamma^{2}}\right) \sum_{i=0}^{j}\left\|T_{i, k+1} \sigma_{\infty}\right\|_{2}^{2}\right. \\
\left.+\frac{1}{\gamma^{2}} \sum_{i=0}^{j-1}\left\|T_{i, k+1} \phi_{\infty}\right\|_{2}^{2}+\frac{1}{\gamma^{2}}\left(1-\delta_{j 0}\right)\left\|\partial_{t} T_{j-1, k} \sigma_{\infty}\right\|_{2}^{2}+\frac{1}{\nu^{2}} \sum_{i=0}^{j-1} D^{(0)}\left[T_{i, k} w_{\infty}\right]\right\},
\end{gathered}
$$

where $\delta_{j 0}$ denotes Kronecker's delta and $R_{j, k}^{(1)}$ is given by 


$$
\begin{gathered}
R_{j, k}^{(1)}=\frac{\alpha_{0}}{\gamma^{2}}\left(\left[Q_{0} T_{j, k} \boldsymbol{F}\right]_{\infty}, T_{j, k} \sigma_{\infty}\right)-\frac{\alpha_{0}}{\gamma^{2}}\left(\left[Q_{0} T_{j, k}(\mathbb{P} \boldsymbol{F})\right]_{\infty}, T_{j, k} \sigma_{\infty}\right)+\widetilde{R}_{j, k}^{(1)}-\left\langle T_{j, k}\left(\left[Q_{0} \boldsymbol{F}\right]_{\infty} u^{(0)}\right), T_{j, k} u_{\infty}\right\rangle_{\Omega} \\
-\left\langle T_{j, k}(\mathbb{P} \boldsymbol{F}), T_{j, k} u_{\infty}\right\rangle_{\Omega}+\left\langle T_{j, k}\left(\left[Q_{0}(\mathbb{P} \boldsymbol{F})\right]_{\infty} u^{(0)}\right), T_{j, k} u_{\infty}\right\rangle_{\Omega}
\end{gathered}
$$

Here,

$$
\widetilde{R}_{j, k}^{(1)}=\left\langle T_{j, k} \boldsymbol{F}, T_{j, k} u_{\infty}\right\rangle_{\Omega}
$$

when $2 j+k \leq m-1$, and

$$
\begin{gathered}
\widetilde{R}_{j, k}^{(1)}=-\left(T_{j, k}(\phi \operatorname{div} w), T_{j, k} \phi_{\infty} \frac{P^{\prime}\left(\rho_{p}\right)}{\gamma^{4} \rho_{p}}\right)+\frac{1}{2}\left(\operatorname{div}\left(\frac{P^{\prime}\left(\rho_{p}\right)}{\gamma^{4} \rho_{p}} w\right),\left|T_{j, k} \phi_{\infty}\right|^{2}\right) \\
-\left(w \nabla T_{j k}\left(\sigma_{*} \phi^{(0)}+\phi_{1}\right), T_{j k} \phi_{\infty} \frac{P^{\prime}\left(\rho_{p}\right)}{\gamma^{4} \rho_{p}}\right)-\left(\left[T_{j, k}, w\right] \nabla \phi, T_{j, k} \phi_{\infty} \frac{P^{\prime}\left(\rho_{p}\right)}{\gamma^{4} \rho_{p}}\right) \\
+\left(T_{j, k} \boldsymbol{f}_{0}, T_{j, k} w_{\infty} \rho_{p}\right)+\sum_{l=1}^{3}\left\langle T_{j, k} \boldsymbol{f}_{l}, T_{j, k} w_{\infty} \rho_{p}\right\rangle_{-1},
\end{gathered}
$$

when $2 j+k=m$. Here and in what follows, for $G=g+\partial_{x_{j}} \widetilde{g}$ with $g, \widetilde{g} \in L^{2}$ and $v \in H_{0}^{1},\langle G, v\rangle_{-1}$ denotes

$$
\langle G, v\rangle_{-1}=(g, v)-\left(\widetilde{g}, \partial_{x_{j}} v\right)
$$

Proof. We apply $T_{j, k}$ to (5.2) and (5.3). We then take the inner products of the resulting equations with $T_{j, k} \sigma_{\infty}$ and $T_{j, k} u_{\infty}$, respectively. Integration by parts together with symmetric properties of $A$ and $B$ gives us the desired result in the same manner as in [7, Proposition 7.4].

We next derive the $H^{1}$-parabolic estimates for $w_{\infty}$. We define $J\left[\widetilde{P}_{\infty} u\right]$ by

$$
J\left[\widetilde{P}_{\infty} u\right]=-2\left\langle\sigma_{\infty} u^{(0)}+u_{\infty}, B \widetilde{Q} u_{\infty}\right\rangle_{\Omega} \text { for } \widetilde{P}_{\infty} u=\sigma_{\infty} u^{(0)}+u_{\infty} .
$$

A direct computation shows that if $\gamma^{2} \geq 1$ then

for some constant $b_{0}>0$.

$$
\left|J\left[\widetilde{P}_{\infty} u\right]\right| \leq \frac{b_{0} \gamma^{2}}{\nu} E^{(0)}\left[\widetilde{P}_{\infty}\right]+\frac{1}{2} D^{(0)}\left[w_{\infty}\right]
$$

Let $b_{1}$ be a positive constant (to be determined later) and define $E^{(1)}\left[\widetilde{P}_{\infty} u\right]$ by

$$
E^{(1)}\left[\widetilde{P}_{\infty} u\right]=\frac{2 b_{1} \gamma^{2}}{\nu} E^{(0)}\left[\widetilde{P}_{\infty} u\right]+D^{(0)}\left[w_{\infty}\right]+J\left[\widetilde{P}_{\infty} u\right]
$$

Note that if $b_{1} \geq b_{0}$ then $E^{(1)}\left[\widetilde{P}_{\infty} u\right]$ is equivalent to $E^{(0)}\left[\widetilde{P}_{\infty} u\right]+D^{(0)}\left[w_{\infty}\right]$.

Proposition 7.4 There exists $b_{1} \geq \max \left\{b_{0}, 8 C_{0}\right\}$ such that if $\nu \geq \nu_{3}, \gamma^{2} \geq 1$ and $\frac{\gamma^{2}}{\nu+\widetilde{\nu}} \geq \max \left\{1, \gamma_{2}^{2}\right\}$ then the following estimate holds for $0 \leq 2 j+k \leq m-1$,

$$
\begin{gathered}
\frac{1}{2} \frac{d}{d t} E^{(1)}\left[T_{j, k} \widetilde{P}_{\infty} u\right]+\frac{b_{1} \gamma^{2}}{\nu} \frac{3}{4} D^{(0)}\left[T_{j, k} w_{\infty}\right]+\frac{1}{2}\left\|\sqrt{\rho_{p}} T_{j, k} \partial_{t} w_{\infty}\right\|_{2}^{2} \leq R_{j, k}^{(2)} \\
+C \sum_{i=0}^{j}\left\{\left(\frac{\nu^{2}}{\gamma^{4}}+\frac{1}{\nu}\right)\left\|T_{i, k} \phi_{\infty}\right\|_{2}^{2}+\left(\frac{\nu+\widetilde{\nu}}{\gamma^{2}}+\frac{1}{\nu}\right)\left\|T_{i, k+1} \sigma_{\infty}\right\|_{2}^{2}+\frac{1}{\nu}\left\|T_{i, k+1} \phi_{\infty}\right\|_{2}^{2}\right. \\
\left.+\frac{1}{\nu}\left(1-\delta_{j 0}\right)\left\|\partial_{t} T_{j-1, k} \sigma_{\infty}\right\|_{2}^{2}\right\}+C_{0} \frac{\gamma^{2}}{\nu}\left(1+\frac{C}{\nu^{2}}\right) \sum_{i=0}^{j-1} D^{(0)}\left[T_{i, k} w_{\infty}\right] .
\end{gathered}
$$

where

$$
R_{j, k}^{(2)}=\frac{2 b_{1} \gamma^{2}}{\nu} R_{j, k}^{(1)}+C\left\|T_{j, k} \boldsymbol{F}\right\|_{2}^{2}
$$

Proof. We apply $T_{j, k}$ to (5.3) and take inner product with $\partial_{t} T_{j, k} \widetilde{Q} u_{\infty}$ to obtain the desired result in the same manner as in [7, Proposition 7.5]. 
As for the disipative estimates for $x_{n}$-derivatives of $\phi_{\infty}$, we have the following inequality.

Proposition 7.5 The following estimate holds for $0 \leq 2 j+k+l \leq m-1$ :

$$
\frac{1}{2} \frac{d}{d t} \frac{1}{\gamma^{2}}\left\|\sqrt{\frac{P^{\prime}\left(\rho_{p}\right)}{\gamma^{2} \rho_{p}}} T_{j, k} \partial_{x_{n}}^{l+1} \phi_{\infty}\right\|_{2}^{2}+\frac{1}{2(\nu+\widetilde{\nu})}\left\|\frac{P^{\prime}\left(\rho_{p}\right)}{\gamma^{2}} \partial_{x_{n}}^{l+1} T_{j, k} \phi_{\infty}\right\|_{2}^{2} \leq R_{j, k, l}^{(3)}+C \frac{\nu+\widetilde{\nu}}{\gamma^{4}}\left\|K_{j, k, l}\right\|_{2}^{2},
$$

where

$$
R_{j, k, l}^{(3)}=\left|\frac{1}{2}\left(\operatorname{div}\left(\frac{P^{\prime}\left(\rho_{p}\right)}{\gamma^{4} \rho_{p}} w\right),\left|T_{j, k} \partial_{x_{n}}^{l+1} \phi_{\infty}\right|^{2}\right)\right|+C \frac{\nu+\widetilde{\nu}}{\gamma^{4}}\left\|H_{j, k, l}\right\|_{2}^{2}
$$

with

$$
\left\|H_{j, k, l}\right\|_{2}^{2} \leq C\left\{\left\|\left[T_{j, k} \partial_{x_{n}}^{l+1}, w\right] \cdot \nabla \phi_{\infty}\right\|_{2}^{2}+\left\|T_{j, k} \partial_{x_{n}}^{l+1}\left(\widetilde{Q_{0} P_{\infty} \boldsymbol{F}}\right)\right\|_{2}^{2}+\left\|\frac{\gamma^{2} \rho_{p}^{2}}{\nu+\widetilde{\nu}} T_{j, k} \partial_{x_{n}}^{l}\left(Q_{n} P_{\infty} \boldsymbol{F}\right)\right\|_{2}^{2}\right\}
$$

and

$$
\widetilde{Q_{0} P_{\infty} \boldsymbol{F}}=-\phi \operatorname{div} w-w \cdot \nabla\left(\sigma_{*} \phi^{(0)}+\phi_{1}\right)-\left\{Q_{0} \mathbb{P} \boldsymbol{F}+\left[Q_{0} P_{\infty}^{(0)} \boldsymbol{F}\right]_{\infty} \phi^{(0)}\right\}
$$

Here, $K_{j, k, l}$ is estimated as

$$
\begin{gathered}
\frac{\nu+\widetilde{\nu}}{\gamma^{4}}\left\|K_{j, k, l}\right\|_{2}^{2} \leq C \frac{\nu+\widetilde{\nu}}{\gamma^{2}}\left\{\frac{\nu^{2}}{\nu+\widetilde{\nu}}\left\|T_{j, k+1} \partial_{x_{n}}^{l} \partial_{x} w_{\infty}\right\|_{2}^{2}+\frac{1}{\nu+\widetilde{\nu}}\left\|\sqrt{\rho_{p}} T_{j, k} \partial_{x_{n}}^{l} \partial_{t} w_{\infty}\right\|_{2}^{2}\right. \\
+\frac{\nu^{2}}{\gamma^{2}}\left(\sum_{q=0}^{l-1}\left\|T_{j, k+1} \partial_{x_{n}}^{q} \partial_{x} w_{\infty}\right\|_{2}^{2}+\sum_{q=0}^{l}\left\|T_{j, k} \partial_{x_{n}}^{q} \partial_{x} w_{\infty}\right\|_{2}^{2}+\sum_{i=0}^{j}\left\|T_{i, k+1} w_{\infty}\right\|_{2}^{2}\right) \\
+\frac{1}{\nu+\widetilde{\nu}} \sum_{i=0}^{j} \sum_{q=0}^{l}\left\|T_{i, k+1} \partial_{x_{n}}^{q} w_{\infty}\right\|_{2}^{2} \\
\left.+\frac{1}{\gamma^{2}}\left(\sum_{i=0}^{j} \sum_{q=0}^{l+1}\left\|T_{i, k+1} \partial_{x_{n}}^{q} \phi_{\infty}\right\|_{2}^{2}+\sum_{i=0}^{j}\left\|T_{i, k+1} \sigma_{\infty}\right\|_{2}^{2}+\sum_{q=0}^{l}\left\|\partial_{x_{n}}^{q} T_{j, k} \phi_{\infty}\right\|_{2}^{2}\right)\right\} .
\end{gathered}
$$

Proof. We obtain the desired result in the same manner as in [7, Proposition 7.6].

The following estimate for the material derivative of $\phi_{\infty}$ plays an important role to obtain the dissipative estimate for higher order $x_{2}$-derivatives of $w_{\infty}$. We denote the material derivative of $\phi_{\infty}$ by $\dot{\phi}_{\infty}$ :

$$
\dot{\phi}_{\infty}=\partial_{t} \phi_{\infty}+\left(v_{p}+w\right) \cdot \nabla \phi_{\infty}
$$

Proposition 7.6 The following estimates hold for $0 \leq 2 j+k+l \leq m-1$ :

$$
\begin{gathered}
\frac{1}{2} \frac{d}{d t} \frac{1}{\gamma^{2}}\left\|\sqrt{\frac{P^{\prime}\left(\rho_{p}\right)}{\gamma^{2} \rho_{p}}} T_{j, k} \partial_{x_{n}}^{l+1} \phi_{\infty}\right\|_{2}^{2}+\frac{1}{4(\nu+\widetilde{\nu})}\left\|\frac{P^{\prime}\left(\rho_{p}\right)}{\gamma^{2}} \partial_{x_{n}}^{l+1} T_{j, k} \phi_{\infty}\right\|_{2}^{2} \\
+c_{0} \frac{\nu+\widetilde{\nu}}{\gamma^{4}}\left\|T_{j, k} \partial_{x_{n}}^{l+1} \dot{\phi}_{\infty}\right\|_{2}^{2} \leq R_{j, k, l}^{(3)}+C \frac{\nu+\widetilde{\nu}}{\gamma^{4}}\left\|K_{j, k, l}\right\|_{2}^{2},
\end{gathered}
$$

where $c_{0}$ is a positive constant and $R_{j, k, l}^{(3)}$ and $K_{j, k, l}$ satisfy the same estimates as in Proposition 7.5.

(ii) Let $0 \leq q \leq k$ and $0 \leq 2 j+k \leq m$. Then

$$
\begin{gathered}
\frac{\nu+\widetilde{\nu}}{\gamma^{4}}\left\|T_{j, k} \dot{\phi}_{\infty}\right\|_{2}^{2} \leq C\left\{R_{j, k}^{(4)}+D^{(0)}\left[T_{j, k} w_{\infty}\right]+\frac{\nu^{2}(\nu+\widetilde{\nu})}{\gamma^{4}}\left\|T_{j, k} w_{\infty}\right\|_{2}^{2}\right. \\
\left.+\frac{\nu+\widetilde{\nu}}{\gamma^{4}} \sum_{i=0}^{j}\left\|T_{i, k+1} \sigma_{\infty}\right\|_{2}^{2}+\frac{\nu+\widetilde{\nu}}{\gamma^{4}} \sum_{i=0}^{j}\left\|T_{i, q} \phi_{\infty}\right\|_{2}^{2}\right\}
\end{gathered}
$$

where $R_{j, k}^{(4)}=\frac{\nu+\widetilde{\nu}}{\gamma^{4}}\left\|T_{j, k} \widehat{Q_{0} P_{\infty} \boldsymbol{F}}\right\|_{2}^{2}$. 
Proof. The desired result is obtained in the same manner as in [7, Proposition 7.7].

Let us derive the dissipative estimates for $\sigma_{\infty}$.

Proposition 7.7 Let $\gamma^{2} /(\nu+\widetilde{\nu}) \geq \max \left\{1, \gamma_{2}^{2}\right\}$, then there holds the following estimate for $0 \leq 2 j+k \leq m-1$ :

$$
\begin{gathered}
\frac{1}{2} \frac{d}{d t} \frac{\nu}{\gamma^{2}(\nu+\widetilde{\nu})}\left\|T_{j, k} \sigma_{\infty}\right\|_{2}^{2}+\frac{\alpha_{1}}{2(\nu+\widetilde{\nu})}\left\|\nabla^{\prime} T_{j, k} \sigma_{\infty}\right\|_{2}^{2} \leq R_{j, k}^{(5)} \\
+C \frac{\nu^{2}}{\gamma^{4}(\nu+\widetilde{\nu})}\left(1-\delta_{j 0}\right)\left\|\partial_{t} T_{j-1, k} \sigma_{\infty}\right\|_{2}^{2}+\frac{1}{\nu+\widetilde{\nu}}\left(\frac{\alpha_{1}}{16}+C \frac{\nu+\widetilde{\nu}}{\gamma^{2}}\right) \sum_{i=0}^{j-1}\left\|T_{i, k+1} \sigma_{\infty}\right\|_{2}^{2} \\
+C\left\{\frac{1}{\nu+\widetilde{\nu}}\left\|\sqrt{\rho_{p}} T_{j, k} \partial_{t} w_{\infty}\right\|_{2}^{2}+\sum_{i=0}^{j} D^{(0)}\left[T_{i, k} w_{\infty}\right]+\frac{1}{\nu+\widetilde{\nu}} \sum_{i=0}^{j}\left\|\frac{P^{\prime}\left(\rho_{p}\right)}{\gamma^{2}} T_{i, p} \partial_{x_{n}} \phi_{\infty}\right\|_{2}^{2}\right\},
\end{gathered}
$$

where $\alpha_{1}>0$ is a constant, $p$ is any integer satisfying $0 \leq p \leq k$, and

$$
R_{j, k}^{(5)}=\frac{\nu}{\gamma^{2}(\nu+\widetilde{\nu})}\left(Q_{0} T_{j, k}\left(P_{\infty}^{(0)} \boldsymbol{F}\right), T_{j, k} \sigma_{\infty}\right)-\frac{1}{\nu+\widetilde{\nu}}\left(\operatorname{div}^{\prime}\left[\rho_{p}(-\Delta)^{-1}\left(\rho_{p} T_{j, k} Q^{\prime}\left(P_{\infty} \boldsymbol{F}\right)\right)\right]_{\infty}, T_{j, k} \sigma_{\infty}\right)
$$

Here, $(-\Delta)^{-1}$ is the inverse of $-\Delta$ on $L^{2}(\Omega)$ with domain $D(-\Delta)=H^{2}(\Omega) \cap H_{0}^{1}(\Omega)$.

Proof. The desired result is obtained in the same manner as in [7, Proposition 7.8].

Next, we estimate the higher order derivatives.

Proposition 7.8 If $\nu \geq 1$ then there holds the following estimate for $0 \leq 2 j+k+l \leq m-1$ :

$$
\begin{gathered}
\frac{\nu^{2}}{\nu+\widetilde{\nu}}\left\|\partial_{x}^{l+2} T_{j, k} w_{\infty}\right\|_{2}^{2}+\frac{1}{\nu+\widetilde{\nu}}\left\|\partial_{x}^{l+1} T_{j, k} \phi_{\infty}\right\|_{2}^{2} \leq C R_{j, k, l}^{(6)}+C\left\{\left(\frac{1}{\nu+\widetilde{\nu}}+\frac{\nu+\widetilde{\nu}}{\gamma^{4}}\right) \sum_{i=0}^{j}\left\|T_{i, k+1} \sigma_{\infty}\right\|_{2}^{2}\right. \\
+\frac{\nu+\widetilde{\nu}}{\gamma^{4}} \sum_{i=0}^{j}\left\|T_{i, k} \phi_{\infty}\right\|_{H^{l}}^{2}+\frac{\nu+\widetilde{\nu}}{\gamma^{4}}\left\|T_{j, k} \dot{\phi}_{\infty}\right\|_{H^{l+1}}^{2}+\frac{1}{\nu+\widetilde{\nu}}\left\|\partial_{t} T_{j, k} w_{\infty}\right\|_{H^{l}}^{2} \\
\left.+\left(\frac{1}{\nu+\widetilde{\nu}}+\frac{\nu^{2}(\nu+\widetilde{\nu})}{\gamma^{4}}\right) \sum_{i=0}^{j}\left\|T_{i, k} w_{\infty}\right\|_{H^{l+1}}^{2}+D^{(0)}\left[T_{j, k} w_{\infty}\right]\right\}
\end{gathered}
$$

where

$$
R_{j, k, l}^{(6)}=\frac{\nu+\widetilde{\nu}}{\gamma^{4}}\left\|T_{j, k} \widehat{Q_{0} P_{\infty} \boldsymbol{F}}\right\|_{H^{l+1}}^{2}+\frac{1}{\nu+\widetilde{\nu}}\left\|T_{j, k}\left(\widetilde{Q} P_{\infty} \boldsymbol{F}\right)\right\|_{H^{l}}^{2}
$$

Proof. We use the estimates for the Stokes system. Let ${ }^{T}(\widetilde{\phi}, \widetilde{w})$ be the solution of the Stokes system

$$
\begin{gathered}
\operatorname{div} \widetilde{w}=F \text { in } \Omega, \\
-\Delta \widetilde{w}-\nabla \widetilde{\phi}=G \text { in } \Omega, \\
\left.\widetilde{w}\right|_{\delta \Omega}=0 .
\end{gathered}
$$

Then for any $l \in \mathbb{Z}, l \geq 0$, there exists a constant $C>0$ such that

$$
\left\|\partial_{x}^{l+2} \widetilde{w}\right\|_{2}^{2}+\left\|\partial_{x}^{l+1} \widetilde{\phi}\right\|_{2}^{2} \leq C\left\{\|F\|_{H^{l+1}}^{2}+\|G\|_{H^{l}}^{2}+\left\|\partial_{x} \widetilde{w}\right\|_{2}^{2}\right\}
$$

(see, e.g., [3][4, Appendix]).

We rewrite (5.3) in the form of Stokes system with $\widetilde{w}=T_{j, k} w_{\infty}$ and $\widetilde{\phi}=\frac{P^{\prime}\left(\rho_{p}\right)}{\nu \gamma^{2}} T_{j, k} \phi_{\infty}$. The desired result is then obtained by using (7.9) (cf. [7, Proposition 7.9]).

At last we estimate the time derivatives of $\sigma_{\infty}$ and $\phi_{\infty}$. 
Proposition 7.9 (i) If $0 \leq 2 j+k \leq m-1$, then there holds the following estimate:

$$
\left\|\partial_{t} T_{j, k} \sigma_{\infty}\right\|_{2}^{2} \leq C\left\{R_{j, k}^{(7)}+\sum_{i=0}^{j}\left(\left\|T_{i, k+1} \sigma_{\infty}\right\|_{2}^{2}+\left\|T_{i, k+1} \phi_{\infty}\right\|_{2}^{2}\right)+\gamma^{4}\left\|T_{j, k+1} w_{\infty}\right\|_{2}^{2}\right\},
$$

Here, $R_{j, k}^{(7)}=\left\|\left[Q_{0} T_{j, k}\left(P_{\infty}^{(0)} \boldsymbol{F}\right)\right]_{\infty}\right\|_{2}^{2}$.

(ii) If $0 \leq k+2 j \leq m-1$ then there holds the following estimate:

$$
\left\|\partial_{t}^{j+1} \phi_{\infty}\right\|_{H^{k}}^{2} \leq C\left\{R_{j}^{(8)}+\sum_{i=0}^{j}\left(\left\|\partial_{x^{\prime}} \partial_{t}^{i} \phi_{\infty}\right\|_{H^{k}}^{2}+\left\|\partial_{x^{\prime}} \partial_{t}^{i} \sigma_{\infty}\right\|_{2}^{2}\right)+\gamma^{4}\left\|\partial_{x} \partial_{t}^{j} w_{\infty}\right\|_{H^{k}}^{2}\right\}
$$

Here, $R_{j, k}^{(8)}=\left\|\partial_{t}^{j}\left(Q_{0} P_{\infty} \boldsymbol{F}\right)\right\|_{H^{k}}^{2}$.

Proof. The estimates (7.10) and (7.11) follow from (5.2) and the first line of (5.3).

Proposition 7.1 now follows from combination of results in Propositions 7.3-7.9.

Proof of Proposition 7.1. Let us define

$$
\begin{gathered}
\widetilde{E}^{(0)}(t)=\sum_{\substack{2 j+k \leq m \\
2 j \neq m}} E^{(0)}\left[T_{j, k} \widetilde{P}_{\infty} u(t)\right], \quad \widetilde{E}^{(1)}(t)=\sum_{2 j+k \leq m-1} E^{(1)}\left[T_{j, k} \widetilde{P}_{\infty} u(t)\right], \\
E^{(2)}(t)=\sum_{2 j+k \leq m-1} \frac{1}{\gamma^{2}}\left\|\sqrt{\frac{P^{\prime}\left(\rho_{p}\right)}{\gamma^{2} \rho_{p}}} \partial_{x_{n}} T_{j, k} \phi_{\infty}(t)\right\|_{2}^{2}, \quad E^{(3)}(t)=\sum_{2 j+k \leq m-1} \frac{\nu}{\gamma^{2}(\nu+\widetilde{\nu})}\left\|T_{j, k} \sigma_{\infty}(t)\right\|_{2}^{2},
\end{gathered}
$$

and

$$
\begin{gathered}
\widetilde{D}^{(0)}(t)=\sum_{\substack{2 j+k \leq m \\
2 j \neq m}} D^{(0)}\left[T_{j, k} w_{\infty}(t)\right], \\
D^{(1)}(t)=\sum_{2 j+k \leq m-1}\left(\frac{3 b_{1} \gamma^{2}}{2 \nu(\nu+\widetilde{\nu})} D^{(0)}\left[T_{j, k} w_{\infty}(t)\right]+\frac{1}{\nu+\widetilde{\nu}}\left\|\sqrt{\rho_{p}} T_{j, k} \partial_{t} w_{\infty}(t)\right\|_{2}^{2}\right), \\
D^{(2)}(t)=\sum_{2 j+k \leq m-1}\left(\frac{1}{2(\nu+\widetilde{\nu})}\left\|\frac{P^{\prime}\left(\rho_{p}\right)}{\gamma^{2}} \partial_{x_{n}} T_{j, k} \phi_{\infty}(t)\right\|_{2}^{2}+\min \left\{1,2 c_{0}\right\} \frac{\nu+\widetilde{\nu}}{\gamma^{4}}\left\|T_{j, k} \dot{\phi}_{\infty}(t)\right\|_{H^{1}}^{2}\right), \\
D^{(3)}(t)=\sum_{2 j+k \leq m-1} \frac{\alpha_{1}}{\nu+\widetilde{\nu}}\left\|\nabla^{\prime} T_{j, k} \sigma_{\infty}(t)\right\|_{2}^{2} .
\end{gathered}
$$

Let $b_{l}, l=2, \ldots, 5$, be positive numbers and let us consider

$$
\begin{gathered}
\sum_{2 j+k \leq m \mid 2 j \neq m}\left\{2 \times(7.2)+2 b_{2} \times(7.6)\right\} \\
+\sum_{2 j+k \leq m-1}\left\{\frac{2}{\nu+\widetilde{\nu}} \times(7.3)+2 b_{2} \times(7.5)_{l=0}+2 b_{3} \times(7.7)+b_{4} \times(7.8)_{l=0}\right\} \\
+\sum_{2 j+k \leq m-1} \frac{1}{(\nu+\widetilde{\nu}) \gamma^{2}} b_{5} \times((7.10)+(7.11))+2 \frac{b_{6}}{\nu+\widetilde{\nu}} \times(7.2)_{2 j=m} .
\end{gathered}
$$

Then we obtain

$$
\frac{d}{d t} E^{(4)}(t)+D^{(4)}(t)+\frac{1}{(\nu+\widetilde{\nu}) \gamma^{2}} b_{5} \sum_{2 j+k \leq m-1}\left(\left\|\partial_{t} T_{j, k} \sigma_{\infty}\right\|_{2}^{2}+\left\|\partial_{t}^{j+1} \phi_{\infty}(t)\right\|_{k}^{2}\right)
$$




$$
\leq \sum_{j=1}^{8} R^{(j)}(t)+R H S
$$

Here,

$$
\begin{gathered}
E^{(4)}(t)=\widetilde{E}^{(0)}+\frac{1}{\nu+\widetilde{\nu}} \widetilde{E}^{(1)}(t)+b_{2} E^{(2)}(t)+b_{3} E^{(3)}(t)+\frac{b_{6}}{\nu+\widetilde{\nu}} E^{(0)}\left[\partial_{t}^{\left[\frac{m}{2}\right]} \widetilde{P}_{\infty} u(t)\right], \\
D^{(4)}=\widetilde{D}^{(0)}(t)+D^{(1)}(t)+b_{2} D^{(2)}(t)+b_{3} D^{(3)}(t)+b_{4} \sum_{2 j+k \leq m-1}\left(\frac{\nu^{2}}{\nu+\widetilde{\nu}}\left\|\partial_{x}^{2} T_{j, k} w_{\infty}\right\|_{2}^{2}+\frac{1}{\nu+\widetilde{\nu}}\left\|\partial_{x} T_{j, k} \phi_{\infty}\right\|_{2}^{2}\right) \\
+\frac{b_{6}}{\nu+\widetilde{\nu}} D^{(0)}\left[\partial_{t}^{\left[\frac{m}{2}\right]} w_{\infty}\right],
\end{gathered}
$$

and

$$
\begin{gathered}
R^{(1)}=\sum_{2 j+k \leq m} R_{j, k}^{(1)}, R^{(p)}=\sum_{2 j+k \leq m-1} R_{j, k}^{(p)}, p=2,5,7,8, R^{(4)}=\sum_{\substack{2 j+k \leq m \\
2 j \neq m}} R_{j, k}^{(4)}, \\
R^{(p)}=\sum_{2 j+k \leq m-1} R_{j, k, 0}^{(p)}, p=3,6,
\end{gathered}
$$

with

$$
\begin{gathered}
R H S=C\left(\frac{\nu+\widetilde{\nu}}{\gamma^{2}}+\frac{1}{\nu}\right) \frac{1}{\nu+\widetilde{\nu}} \sum_{2 j+k \leq m-1}\left\|\partial_{x} T_{j, k} \phi_{\infty}\right\|_{2}^{2}+C\left(\frac{\nu+\widetilde{\nu}}{\gamma^{2}}+\frac{1}{\nu}+b_{4}\right) \frac{1}{\nu+\widetilde{\nu}} \sum_{2 j+k \leq m-1}\left\|\partial_{x^{\prime}} T_{j, k} \sigma_{\infty}\right\|_{2}^{2} \\
+C\left(\frac{1}{\gamma^{2}}+\frac{1}{\nu(\nu+\widetilde{\nu})}\right) \sum_{2 j+k \leq m-2}\left\|\partial_{t} T_{j, k} \sigma_{\infty}\right\|_{2}^{2}+C\left(\frac{1}{\nu^{2}}+b_{2}+b_{3}+b_{4}\right) \sum_{2 j+k \leq m \mid 2 j \neq m} D^{(0)}\left[T_{j, k} w_{\infty}\right] \\
+\left(\frac{b_{1}}{4}+b_{5} C\right) \frac{\gamma^{2}}{\nu(\nu+\widetilde{\nu})} \sum_{2 j+k \leq m-1} D^{(0)}\left[T_{j, k} w_{\infty}\right]+C\left(b_{2}+b_{3}+b_{4}\right) \sum_{2 j+k \leq m-1} \frac{1}{\nu+\widetilde{\nu}}\left\|\sqrt{\rho_{p}} T_{j, k} \partial_{t} w_{\infty}\right\|_{2}^{2} \\
+\frac{b_{3} \alpha_{1}}{4(\nu+\widetilde{\nu})} \sum_{2 j+k \leq m-3}\left\|T_{j, k+1} \sigma_{\infty}\right\|_{2}^{2}+C b_{3} \frac{1}{\nu+\widetilde{\nu}} \sum_{2 j+k \leq m-1}\left\|\frac{P^{\prime}\left(\rho_{p}\right)}{\gamma^{2}} \partial_{x_{n}} T_{j, k} \phi_{\infty}\right\|_{2}^{2} \\
+C b_{4} \frac{\nu+\widetilde{\nu}}{\gamma^{4}} \sum_{2 j+k \leq m-1}\left\|T_{j, k} \dot{\phi}_{\infty}\right\|_{H^{1}}^{2}+C b_{6} \frac{1}{(\nu+\widetilde{\nu}) \gamma^{2}}\left(\left\|\partial_{t}^{\left[\frac{m}{2}\right]} \phi_{\infty}\right\|_{2}^{2}+\left\|\partial_{t}^{\left[\frac{m}{2}\right]} \sigma_{\infty}\right\|_{2}^{2}\right) .
\end{gathered}
$$

There exists $\nu_{0} \geq \max \left\{1, \nu_{3}\right\}, \gamma_{0} \geq \max \left\{1, \gamma_{2}\right\}$ and $1>b>0$ such that if $b_{4}<b_{3}<b_{2}$ and $b_{6} \leq b_{5} \leq b_{1}$ appropriately with $b_{l} \leq b$ for $l=2, \ldots, 4$, and $\nu \geq \nu_{0}$ and $\gamma^{2} /(\nu+\widetilde{\nu}) \geq \gamma_{0}^{2}$ we can absorb most of the terms from RHS in the left-hand side of (7.12) to get

$$
\begin{gathered}
\frac{d}{d t} E^{(4)}(t)+\frac{1}{2} D^{(4)}(t)+\frac{1}{2} \frac{1}{(\nu+\widetilde{\nu}) \gamma^{2}} b_{5} \sum_{2 j+k \leq m-1}\left\|\partial_{t} T_{j, k} \sigma_{\infty}\right\|_{2}^{2}+\frac{1}{2} \frac{1}{(\nu+\widetilde{\nu}) \gamma^{2}} b_{5} \sum_{2 j \leq m-2}\left\|\partial_{t}^{j+1} \phi_{\infty}(t)\right\|_{2}^{2} \\
\leq C \sum_{j=1}^{8} R^{(j)}(t)+C \sum_{2 j+k \leq m-2}\left\|\partial_{t} T_{j, k} \sigma_{\infty}\right\|_{2}^{2} \frac{1}{\nu+\widetilde{\nu}} .
\end{gathered}
$$

Next, we estimate higher order derivatives in $x_{n}$. For $1 \leq l \leq m-1$, we set

$$
E_{l}^{(4)}(t)=\sum_{2 j+k \leq m-1-l} \frac{1}{\gamma^{2}}\left\|\sqrt{\frac{P^{\prime}\left(\rho_{p}\right)}{\gamma^{2}}} T_{j, k} \partial_{x_{n}}^{l+1} \phi_{\infty}(t)\right\|_{2}^{2},
$$

and 


$$
\begin{aligned}
D_{l}^{(4)}(t)= & \sum_{2 j+k \leq m-1-l}\left(\frac{1}{2(\nu+\widetilde{\nu})}\left\|\frac{P^{\prime}\left(\rho_{p}\right)}{\gamma^{2}} \partial_{x_{n}}^{l+1} T_{j, k} \phi_{\infty}(t)\right\|_{2}^{2}+\frac{2 c_{0}(\nu+\widetilde{\nu})}{\gamma^{4}}\left\|T_{j, k} \partial_{x_{n}}^{l+1} \dot{\phi}_{\infty}(t)\right\|_{2}^{2}\right) \\
& +b_{7} \sum_{2 j+k \leq m-1-l}\left(\frac{\nu^{2}}{\nu+\widetilde{\nu}}\left\|\partial_{x}^{l+2} T_{j, k} w_{\infty}(t)\right\|_{2}^{2}+\frac{1}{\nu+\widetilde{\nu}}\left\|\partial_{x}^{l+1} T_{j, k} \phi_{\infty}(t)\right\|_{2}^{2}\right) .
\end{aligned}
$$

We add $2 \times(7.5)$ to $b_{7} \times(7.8)$ and sum over $2 j+k \leq m-1-l$ to obtain

$$
\begin{gathered}
\frac{d}{d t} E_{l}^{(4)}(t)+D_{l}^{(4)}(t) \leq C R_{l}^{(9)}+b_{7} C \frac{\nu+\widetilde{\nu}}{\gamma^{4}} \sum_{2 j+k \leq m-1-l}\left\|T_{j, k} \dot{\phi}_{\infty}\right\|_{H^{l+1}}^{2} \\
+C\left(\frac{\nu+\widetilde{\nu}}{\gamma^{2}}+b_{7}\right) \frac{1}{\nu+\widetilde{\nu}} \sum_{2 j+k \leq m-1-l}\left\|T_{j, k} \partial_{t} w_{\infty}\right\|_{H^{l}}^{2}+C\left(\frac{1}{\nu^{2}}+\frac{\nu+\widetilde{\nu}}{\gamma^{2}}\right) \frac{\nu^{2}}{\nu+\widetilde{\nu}} \sum_{2 j+k \leq m-1-l}\left\|T_{j, k} w_{\infty}\right\|_{H^{l+2}}^{2} \\
+C\left(b_{7}+\frac{\nu+\widetilde{\nu}}{\gamma^{2}}\right) \frac{1}{\nu+\widetilde{\nu}} \sum_{2 j+k \leq m-1-l}\left\|T_{j, k+1} \sigma_{\infty}\right\|_{2}^{2}+C \frac{\nu+\widetilde{\nu}}{\gamma^{2}} \frac{1}{\nu+\widetilde{\nu}} \sum_{2 j+k \leq m-1-l}\left\|T_{j, k} \phi_{\infty}\right\|_{H^{l+1}}^{2} .
\end{gathered}
$$

Here, $R_{l}^{(9)}=\sum_{2 j+k \leq m-1-l}\left(R_{j, k, l}^{(3)}+R_{j, k, l}^{(6)}\right)$.

Let us sum up to $l$ to get

$$
\begin{gathered}
\frac{d}{d t} \sum_{p=1}^{l} E_{p}^{(4)}(t)+\sum_{p=1}^{l} D_{p}^{(4)}(t) \leq C \sum_{p=1}^{l} R_{p}^{(9)} \\
+b_{7} C \frac{\nu+\widetilde{\nu}}{\gamma^{4}} \sum_{p=1}^{l} \sum_{2 j+k \leq m-1-p}\left\|T_{j, k} \dot{\phi}_{\infty}\right\|_{H^{p+1}}^{2}+C\left(\frac{\nu+\widetilde{\nu}}{\gamma^{2}}+b_{7}\right) \frac{1}{\nu+\widetilde{\nu}} \sum_{p=1}^{l} \sum_{2 j+k \leq m-1-p}\left\|T_{j, k} \partial_{t} w_{\infty}\right\|_{H^{p}}^{2} \\
+C\left(\frac{1}{\nu^{2}}+\frac{\nu+\widetilde{\nu}}{\gamma^{2}}\right) \frac{\nu^{2}}{\nu+\widetilde{\nu}} \sum_{p=1}^{l} \sum_{2 j+k \leq m-1-p}\left\|T_{j, k} w_{\infty}\right\|_{H^{p+2}}^{2}+C\left(b_{7}+\frac{\nu+\widetilde{\nu}}{\gamma^{2}}\right) \frac{1}{\nu+\widetilde{\nu}} \sum_{p=1}^{l} \sum_{2 j+k \leq m-1-p}\left\|T_{j, k+1} \sigma_{\infty}\right\|_{2}^{2} \\
+C \frac{\nu+\widetilde{\nu}}{\gamma^{2}} \frac{1}{\nu+\widetilde{\nu}} \sum_{p=1}^{l} \sum_{2 j+k \leq m-1-p}\left\|T_{j, k} \phi_{\infty}\right\|_{H^{p+1}}^{2} .
\end{gathered}
$$

Taking $b_{7}$ appropriately small, $\nu_{0}$ and $\gamma_{0}$ possibly larger (based on $l$ ) we obtain

$$
\begin{gathered}
\frac{d}{d t} \sum_{p=1}^{l} E_{p}^{(4)}(t)+\frac{1}{2} \sum_{p=1}^{l} D_{p}^{(4)}(t) \leq C \sum_{p=1}^{l} R_{p}^{(9)}+b_{7} C \frac{\nu+\widetilde{\nu}}{\gamma^{4}} \sum_{2 j+k \leq m-2}\left\|T_{j, k} \dot{\phi}_{\infty}\right\|_{H^{1}}^{2} \\
+C \frac{1}{\nu^{2}} \frac{\nu^{2}}{\nu+\widetilde{\nu}} \sum_{2 j+k \leq m-1}\left\|T_{j, k} \partial_{x}^{2} w_{\infty}\right\|_{2}^{2}+C \frac{1}{\nu+\widetilde{\nu}} \sum_{2 j+k \leq m \mid 2 j \neq m}\left\|T_{j, k} w_{\infty}\right\|_{H^{1}}^{2}+C\left(b_{7}+\frac{\nu+\widetilde{\nu}}{\gamma^{2}}\right) \frac{1}{\nu+\widetilde{\nu}} D^{(0)}\left[\partial_{t}^{\left[\frac{m}{2}\right]} w_{\infty}\right] \\
+C\left(\frac{1}{\nu^{2}}+\frac{\nu+\widetilde{\nu}}{\gamma^{2}}\right) \frac{\nu^{2}}{\nu+\widetilde{\nu}} \sum_{2 j+k \leq m-2}\left\|\partial_{x}^{2} T_{j, k} w_{\infty}\right\|_{2}^{2}+C\left(\frac{1}{\nu^{2}}+\frac{\nu+\widetilde{\nu}}{\gamma^{2}}\right) \sum_{2 j+k \leq m-2} D^{(0)}\left[T_{j, k} w_{\infty}\right] \\
+C\left(b_{7}+\frac{\nu+\widetilde{\nu}}{\gamma^{2}}\right) \frac{1}{\nu+\widetilde{\nu}} \sum_{2 j+k \leq m-1}\left\|T_{j, k+1} \sigma_{\infty}\right\|_{2}^{2}+C \frac{\nu+\widetilde{\nu}}{\gamma^{2}} \frac{1}{\nu+\widetilde{\nu}} \sum_{2 j+k \leq m-2}\left\|\partial_{x} T_{j, k} \phi_{\infty}\right\|_{2}^{2} .
\end{gathered}
$$

Now adding $2 \times(7.13)$ together with (7.14) and taking possibly $b_{7}$ smaller, $\nu_{0}$ and $\gamma_{0}$ larger we obtain

$$
\begin{gathered}
\frac{d}{d t}\left(2 E^{(4)}(t)+\sum_{p=1}^{l} E_{p}^{(4)}(t)\right)+\left(D^{(4)}(t)+\sum_{p=1}^{l} D_{p}^{(4)}(t)\right) \\
+\frac{1}{(\nu+\widetilde{\nu}) \gamma^{2}} b_{5} \sum_{2 j+k \leq m-1}\left\|\partial_{t} T_{j, k} \sigma_{\infty}\right\|_{2}^{2}+\frac{1}{(\nu+\widetilde{\nu}) \gamma^{2}} b_{5} \sum_{2 j \leq m-2}\left\|\partial_{t}^{j+1} \phi_{\infty}(t)\right\|_{2}^{2}
\end{gathered}
$$




$$
\leq C\left(\sum_{j=1}^{8} R^{(j)}(t)+\sum_{p=1}^{l} R_{p}^{(9)}\right)+C \sum_{2 j+k \leq m-2}\left\|\partial_{t} T_{j, k} \sigma_{\infty}\right\|_{2}^{2} \frac{1}{\nu+\widetilde{\nu}} .
$$

To absorb the last term on the right-hand side we use induction on $m$.

Let $m=1$ then we have from (7.15) that

$$
\begin{aligned}
& \frac{d}{d t}\left(2 E_{1}^{(4)}(t)+\sum_{p=1}^{l} E_{p, 1}^{(4)}(t)\right)+\left(D_{1}^{(4)}(t)+\sum_{p=1}^{l} D_{p, 1}^{(4)}(t)\right) \\
& +\frac{1}{(\nu+\widetilde{\nu}) \gamma^{2}} b_{5}\left\|\partial_{t} \sigma_{\infty}\right\|_{2}^{2} \leq C\left(\sum_{j=1}^{8} R^{(j)}(t)+\sum_{p=1}^{l} R_{p}^{(9)}\right) .
\end{aligned}
$$

Let $m=2$ then

$$
\begin{gathered}
\frac{d}{d t}\left(2 E^{(4)}(t)+\sum_{p=1}^{l} E_{p}^{(4)}(t)\right)+\left(D^{(4)}(t)+\sum_{p=1}^{l} D_{p}^{(4)}(t)\right) \\
+\frac{1}{(\nu+\widetilde{\nu}) \gamma^{2}} b_{5} \sum_{k \leq 1}\left\|\partial_{t} T_{0, k} \sigma_{\infty}\right\|_{2}^{2}+\frac{1}{(\nu+\widetilde{\nu}) \gamma^{2}} b_{5}\left\|\partial_{t}^{1} \phi_{\infty}(t)\right\|_{2}^{2} \leq C\left(\sum_{j=1}^{8} R^{(j)}(t)+\sum_{p=1}^{l} R_{p}^{(9)}\right) \\
+C\left\|\partial_{t} \sigma_{\infty}\right\|_{2}^{2} \frac{1}{\nu+\widetilde{\nu}} .
\end{gathered}
$$

By adding $b_{8} \gamma^{2} \times(7.16)$ to (7.17) with appropriately large $b_{8}>0$ we can absorb $\left\|\partial_{t} \sigma_{\infty}\right\|_{2}^{2} \frac{1}{\nu+\widetilde{\nu}}$ to the left-hand side. It is straightforward to see that this can be done from $m$ to $m+1$. Therefore, we have

$$
\begin{gathered}
C_{1} \frac{d}{d t}\left(2 E^{(4)}(t)+\sum_{p=1}^{l} E_{p}^{(4)}(t)\right)+\left(D^{(4)}(t)+\sum_{p=1}^{l} D_{p}^{(4)}(t)\right)+\frac{1}{(\nu+\widetilde{\nu}) \gamma^{2}} b_{5} \sum_{2 j+k \leq m-1}\left\|\partial_{t} T_{j, k} \sigma_{\infty}\right\|_{2}^{2} \\
+\frac{1}{(\nu+\widetilde{\nu}) \gamma^{2}} b_{5} \sum_{2 j \leq m-2}\left\|\partial_{t}^{j+1} \phi_{\infty}(t)\right\|_{2}^{2} \leq C_{2}\left(\sum_{j=1}^{8} R^{(j)}(t)+\sum_{p=1}^{l} R_{p}^{(9)}\right),
\end{gathered}
$$

with $C_{1}, C_{2}>0$. The desired estimate (7.1) now follows from (7.18) with $l=m-1$.

Estimate (5.11) for $\widetilde{R}(t)$ is given in Proposition 8.1 (ii) below. This concludes the proof.

To prove (5.10) we employ the following lemma.

Lemma 7.10 There exists $\widetilde{r}_{0}=\widetilde{r}_{0}(\nu, \widetilde{\nu}, \gamma)$ such that if $r_{1} \leq \widetilde{r}_{0}$, then there holds the estimate

$$
\left\|\left[Q_{0} P_{\infty, 1}(t) u\right]\right\|_{2} \leq C\left\|\partial_{x^{\prime}}\left(I-\Pi^{(0)}(t)\right) P_{\infty, 1}(t) u\right\|_{2} .
$$

Proof. We set

$$
\mathscr{R}\left(\xi^{\prime}, t\right)=\widehat{\mathscr{Q}}^{(0)}(t)\left(i \xi^{\prime} \widehat{\mathscr{P}}^{(1)}(t)-\left|\xi^{\prime}\right|^{2} \widehat{\mathscr{P}}^{(2)}\left(\xi^{\prime}, t\right)\right)+\left(i \xi^{\prime} \widehat{\mathscr{Q}}^{(1)}(t)-\left|\xi^{\prime}\right|^{2} \widehat{\mathscr{Q}}^{(2)}\left(\xi^{\prime}, t\right)\right) \widehat{\mathscr{P}}_{\xi^{\prime}}(t) .
$$

Since

$$
\begin{gathered}
{\left[Q_{0} \widehat{\chi}_{1}\left(\xi^{\prime}\right)\left(I-\widehat{\mathscr{Q}}_{\xi^{\prime}}(t) \widehat{\mathscr{P}}_{\xi^{\prime}}(t)\right)\right]=\left[Q_{0} \widehat{\chi}_{1}\left(\xi^{\prime}\right)\left(I-\widehat{\mathscr{Q}}^{(0)}(t) \widehat{\mathscr{P}}^{(0)}-\mathscr{R}\left(\xi^{\prime}, t\right)\right)\right]} \\
=-\left[Q_{0} \widehat{\chi}_{1}\left(\xi^{\prime}\right) \mathscr{R}\left(\xi^{\prime}, t\right)\right],
\end{gathered}
$$

we see that

$$
\left[Q_{0} \widehat{P_{\infty, 1} u}(t)\right]=\left[Q_{0} \widehat{\chi}_{1}\left(\xi^{\prime}\right)\left(I-\widehat{\mathscr{Q}}_{\xi^{\prime}}(t) \widehat{\mathscr{P}}_{\xi^{\prime}}(t)\right) \widehat{u}\right]
$$

It then follows that

$$
\left|\left[Q_{0} \widehat{P_{\infty, 1}(t) u}\right]\right|_{2} \leq C\left|\xi^{\prime}\right|\left|\widehat{\chi}_{1} \widehat{u}\right|_{2}
$$




$$
\leq C\left|\xi^{\prime}\right|\left(\widehat{\chi}_{1}\left|\left(I-\Pi^{(0)}(t)\right) \widehat{u}\right|_{2}+\widehat{\chi}_{1}\left|\Pi^{(0)}(t) \widehat{u}\right|_{2}\right) .
$$

Since $\left(P_{\infty, 1}(t)\right)^{2}=P_{\infty, 1}$, we see that

$$
\left|\left[Q_{0} \widehat{P_{\infty, 1}}(t) u\right]\right|_{2} \leq C\left|\xi^{\prime}\right|\left(\left|\left(I-\Pi^{(0)}(t)\right) \widehat{P_{\infty, 1}(t)} u\right|_{2}+\left|\left[Q_{0} \widehat{P_{\infty, 1}(t)} u\right]\right|_{2}\right),
$$

for $\left|\xi^{\prime}\right| \leq r_{1}$. Therefore, there exists a positive number $\widetilde{r}_{0}$ such that if $r_{1} \leq \widetilde{r}_{0}$ then

$$
\left|\left[Q_{0} \widehat{P_{\infty, 1}}(t) u\right]\right|_{2} \leq C\left|\xi^{\prime}\right|\left|\left(I-\Pi^{(0)}(t)\right) \widehat{P_{\infty, 1} u}\right|_{2},
$$

for $\left|\xi^{\prime}\right| \leq r_{1}$, from which we obtain

$$
\left\|\left[Q_{0} P_{\infty, 1}(t) u\right]\right\|_{2} \leq C\left\|\partial_{x^{\prime}}\left(I-\Pi^{(0)}(t)\right) P_{\infty, 1}(t) u\right\|_{2} .
$$

This completes the proof.

Finally, we prove (5.10).

Proof of (5.10). We fix $\nu, \widetilde{\nu}, \gamma$ so that inequality (7.1) in Proposition 7.1 holds true and $\operatorname{set} r_{1}=\min \left\{r_{0}, \widetilde{r}_{0}, 1\right\}$. Then we proceed as in $[7$, Proof of (5.15)] to obtain

$$
\widetilde{E}(t)+\frac{\nu^{2}}{\nu+\widetilde{\nu}} \llbracket \partial_{x_{n}}^{2} w_{\infty}(t) \rrbracket_{m-2}^{2}+\int_{0}^{t} e^{-\widetilde{a}(t-z)} D(z) d z \leq C\left\{e^{-\widetilde{a} t} \widetilde{E}(0)+R^{(10)}(t)+\int_{0}^{t} e^{-\widetilde{a}(t-z)} \widetilde{R}(z) d z\right\} .
$$

Since

$$
R^{(10)}(t) \leq C(1+t)^{-\frac{n+1}{2}} M(t)^{4},
$$

as we show in Proposition 8.1 (i) below, we deduce (5.10) from (7.19), Proposition 7.1 (i) and (7.20). This completes the proof.

\section{Estimates on the nonlinearities}

In this section we estimates the nonlinearities, e.g., we prove (5.11) and (7.20). In this section we assume that $\nu \geq \nu_{2}$ and $\gamma^{2} /(\nu+\widetilde{\nu}) \geq \gamma_{2}^{2}$.

Proposition 8.1 There exists number $\varepsilon_{6}>0$ such that if solution $u(t)$ of $(4.1)$ in $Z^{m}(\tau)$ satisfies $\sup _{0 \leq z \leq t} \llbracket u(z) \rrbracket_{m} \leq$ $\varepsilon_{6}$ and $M(t) \leq 1$ for all $t \in[0, \tau]$, then the following estimates hold for all $t \in[0, \tau]$ with $C>0$ independent of $\tau$.

(i)

$$
\llbracket \widetilde{Q} P_{\infty} \boldsymbol{F}(t) \rrbracket_{m-2} \leq C(1+t)^{-\frac{2 n-1}{4}} M(t)^{2},
$$

(ii)

$$
\widetilde{R}(t) \leq C\left\{(1+t)^{-\frac{3 n}{4}} M(t)^{3}+(1+t)^{-\frac{n-1}{4}} M(t) D_{\infty}(t)\right\} .
$$

To show the estimates in Proposition 8.1 we use the following inequalities.

Lemma 8.2 (i) Let $2 \leq p \leq \infty$ and let $j$ and $k$ be integers satisfying

$$
0 \leq j<k, \quad k>j+n\left(\frac{1}{2}-\frac{1}{p}\right) .
$$

Then there exists a constant $C>0$ such that

$$
\left\|\partial_{x}^{j} f\right\|_{L^{p}\left(\mathbb{R}^{n}\right)} \leq C\|f\|_{L^{2}\left(\mathbb{R}^{n}\right)}^{1-\theta}\left\|\partial_{x}^{k} f\right\|_{L^{2}\left(\mathbb{R}^{n}\right)}^{\theta},
$$

where $\theta=\frac{1}{k}\left(j+\frac{n}{2}-\frac{n}{p}\right)$. 
(ii) Let $2 \leq p \leq \infty$ and let $j$ and $k$ be integers satisfying

$$
0 \leq j<k, \quad k>j+n\left(\frac{1}{2}-\frac{1}{p}\right) .
$$

Then there exists a constant $C>0$ such that

$$
\left\|\partial_{x}^{j} f\right\|_{L^{p}(\Omega)} \leq C\|f\|_{H^{k}(\Omega)} .
$$

(iii) If $f \in H^{n-1}(\Omega)$ and $f=f\left(x^{\prime}\right)$ is independent of $x_{n}$, then

$$
\|f\|_{L^{\infty}(\Omega)} \leq C\|f\|_{L^{2}(\Omega)}^{\frac{1}{2}}\left\|\partial_{x^{\prime}}^{n-1} f\right\|_{L^{2}(\Omega)}^{\frac{1}{2}} .
$$

Proof. The inequality in (i) is a special case of the Galiardo-Nirenberg-Sobolev inequality which can be proved using Fourier transform. Inequality in (ii) can be obtained by (i) and the standard extension argument. As for (iii), since

$$
\|f\|_{L^{p}(\Omega)}=\|f\|_{L^{p}\left(\mathbb{R}^{n-1}\right)}, 1 \leq p \leq \infty,\left\|\partial_{x^{\prime}} f\right\|_{L^{2}(\Omega)}=\left\|\partial_{x^{\prime}} f\right\|_{L^{2}\left(\mathbb{R}^{n-1}\right)},
$$

the inequality is a consequence of (i) with $n=n-1, p=\infty, j=0$ and $k=n-1$.

Lemma 8.3 (i) Let $m$ and $m_{k}, k=1, \ldots, l$ be nonnegative integers and let $\alpha_{k} k=1, \ldots, l$ be multiindeces. Suppose that

$$
m \geq\left[\frac{n}{2}\right]+1,0 \leq\left|\alpha_{k}\right| \leq m_{k} \leq m+\left|\alpha_{k}\right|, k=1, \ldots, l,
$$

and

$$
m_{1}+\cdots+m_{l} \geq(l-1) m+\left|\alpha_{1}\right|+\cdots+\left|\alpha_{l}\right| .
$$

Then there exists a constant $C>0$ such that

$$
\left\|\partial_{x}^{\alpha_{1}} f_{1} \cdots \partial_{x}^{\alpha_{l}} f_{l}\right\|_{2} \leq C \prod_{1 \leq k \leq l}\left\|f_{k}\right\|_{H^{m_{k}}}
$$

(ii) Let $1 \leq k \leq m$. Suppose that $F(x, t, y)$ is a smooth function on $\Omega \times[0, \infty) \times I$, where $I$ is a compact interval in $\mathbb{R}$. Then for $|\alpha|+2 j=k$ there hold

$$
\left\|\left[\partial_{x}^{\alpha} \partial_{t}^{j}, F\left(x, t, f_{1}\right)\right] f_{2}\right\|_{2} \leq\left\{\begin{array}{l}
C_{0}\left(t, f_{1}(t)\right) \llbracket f_{2} \rrbracket_{k-1}+C_{1}\left(t, f_{1}(t)\right)\left\{1+\left\|\left|D f_{1} \|\right|_{m-1}^{\alpha \mid+j-1}\right\}\left\|\left|D f_{1} \|\right|_{m-1} \llbracket f_{2} \rrbracket_{k}\right.\right. \\
C_{0}\left(t, f_{1}(t)\right) \llbracket f_{2} \rrbracket_{k-1}+C_{1}\left(t, f_{1}(t)\right)\left\{1+\left.\left\|D f_{1}\right\|\right|_{m-1} ^{|\alpha|+j-1}\right\}\left\|\left|D f_{1} \|\right|_{m} \llbracket f_{2} \rrbracket_{k-1}\right.
\end{array}\right.
$$

Here

$$
C_{0}\left(t, f_{1}(t)\right)=\sum_{\substack{(\beta, l) \leq(\alpha, j) \\(\beta, l) \neq(0,0)}} \sup _{x}\left|\left(\partial_{x}^{\beta} \partial_{t}^{l} F\right)\left(x, t, f_{1}(x, t)\right)\right|
$$

and

$$
C_{1}\left(t, f_{1}(t)\right)=\sum_{\substack{(\beta, l) \leq(\alpha, j) \\ 1 \leq p \leq j+|\alpha|}} \sup _{x}\left|\left(\partial_{x}^{\beta} \partial_{t}^{l} \partial_{y}^{p} F\right)\left(x, t, f_{1}(x, t)\right)\right| .
$$

(iii) Let $m \geq[n / 2]+1$ then there exist constants $C, C^{\prime}>0$ such that

$$
\left\|f_{1} \cdot f_{2}\right\|_{H^{m}} \leq C\left\|f_{1}\right\|_{H^{m}}\left\|f_{2}\right\|_{H^{m}}
$$

and when $\llbracket f_{1} \rrbracket_{m} \leq 1$,

$$
\llbracket f_{1} \cdot f_{2} \rrbracket_{m} \leq C^{\prime} \llbracket f_{1} \rrbracket_{m} \llbracket f_{2} \rrbracket_{m} .
$$


Proof of previous lemma can be found in $[9,10]$.

We recall that $u(t)$ is decomposed into

$$
u=\sigma_{1} u^{(0)}+u_{1}+\sigma_{\infty} u^{(0)}+u_{\infty}
$$

and we write

$$
\begin{gathered}
\sigma_{*}=\sigma_{1}+\sigma_{\infty}, \quad \phi_{*}=\phi_{1}+\phi_{\infty}, \quad w_{*}=w_{1}+w_{\infty}, \\
u_{*}={ }^{T}\left(\phi_{*}, w_{*}\right)=u_{1}+u_{\infty} .
\end{gathered}
$$

Before investigating the nonlinearities we present some basic estimates.

Lemma 8.4 Let $u(t)={ }^{T}(\phi(t), w(t))=\left(\sigma_{*} u^{(0)}\right)(t)+u_{*}(t)$ be solution of $(4.1)$ in $Z^{m}(\tau)$. The following estimates hold for all $t \in[0, \tau]$ with $C>0$ independent of $\tau$.

(i)

$$
\left\|\sigma_{*}(t)\right\|_{2} \leq C(1+t)^{-\frac{n-1}{4}} M(t)
$$

(ii)

$$
\left\|\left|D \sigma_{*}(t) \|\right|_{m-1}+\llbracket u_{*}(t) \rrbracket_{m} \leq C(1+t)^{-\frac{n+1}{4}} M(t),\right.
$$

(iii)

(iv)

$$
\llbracket \phi(t) \rrbracket_{m}+\llbracket w(t) \rrbracket_{m} \leq C(1+t)^{-\frac{n-1}{4}} M(t),
$$

(v)

$$
\left\|\sigma_{*}(t)\right\|_{\infty} \leq C(1+t)^{-\frac{n}{4}} M(t)
$$

$$
\left\|u_{*}(t)\right\|_{\infty} \leq C(1+t)^{-\frac{n+1}{4}} M(t),
$$

(vi)

$$
\|\phi(t)\|_{\infty}+\|w(t)\|_{\infty} \leq C(1+t)^{-\frac{n}{4}} M(t) .
$$

Proof. Estimates (i), (ii) and (iii) immediately follow from definition of $M(t)$. As for (iv), we see from Lemma 8.2 (iii) and Lemma 5.3 (iii) that

$$
\left\|\sigma_{*}(t)\right\|_{\infty} \leq C\left\|\sigma_{*}(t)\right\|_{2}^{\frac{1}{2}}\left\|\partial_{x^{\prime}}^{n-1} \sigma_{*}(t)\right\|_{2}^{\frac{1}{2}} \leq C\left\|\sigma_{*}(t)\right\|_{2}^{\frac{1}{2}}\left\|\partial_{x^{\prime}} \sigma_{*}(t)\right\|_{2}^{\frac{1}{2}} \leq C(1+t)^{-\frac{n}{4}} M(t) .
$$

This shows (iv). Since $\left\|u_{*}(t)\right\|_{\infty} \leq C\left\|u_{*}(t)\right\|_{H^{m}}$ by Lemma 8.2 (ii) we get get (v) from (ii). Estimate (vi) now follows from (iv) and (v). This completes the proof.

First, we consider the estimates on $Q_{0} \boldsymbol{F}$.

Proposition 8.5 Let $u(t)$ be a solution of $(4.1)$ in $Z^{m}(\tau)$ such that $M(t) \leq 1$ for all $t \in[0, \tau]$. There hold the following estimates for all $t \in[0, \tau]$ with $C>0$ independent of $\tau$.

$$
\llbracket \phi \operatorname{div} w \rrbracket_{l} \leq C \begin{cases}(1+t)^{-\frac{2 n+1}{4}} M(t)^{2}+(1+t)^{-\frac{n}{4}} M(t)\left\|\left|D w_{\infty}(t) \|\right|_{m},\right. & l=m, \\ (1+t)^{-\frac{2 n+1}{4}} M(t)^{2}, & l=m-1,\end{cases}
$$

(ii)

$$
\llbracket w \cdot \nabla\left(\sigma_{*} \phi^{(0)}+\phi_{1}\right) \rrbracket_{m} \leq C(1+t)^{-\frac{2 n+1}{4}} M(t)^{2}
$$

(iii)

$$
\llbracket w \cdot \nabla \phi_{\infty} \rrbracket_{m-1} \leq C(1+t)^{-\frac{2 n+1}{4}} M(t)^{2},
$$

(iv)

$$
\left|\left(\operatorname{div}\left(\frac{P^{\prime}\left(\rho_{p}\right)}{\gamma^{4} \rho_{p}} w\right),\left|\partial_{t}^{j} \partial_{x}^{k} \phi_{\infty}\right|^{2}\right)\right| \leq C(1+t)^{-\frac{n+1}{4}} M(t) D_{\infty}(t)
$$

for $2 j+k \leq m$, 
(v)

$$
\left\|\left[\partial_{t}^{j} \partial_{x}^{k}, w\right] \cdot \nabla \phi\right\|_{2} \leq C(1+t)^{-\frac{n}{2}} M(t)^{2}+(1+t)^{-\frac{n-1}{4}} M(t) \sqrt{D_{\infty}(t)},
$$

for $2 j+k \leq m$,

(vi)

$$
\left\|T_{j, k}(\phi w)\right\|_{2} \leq(1+t)^{-\frac{2 n-1}{4}} M(t)^{2}
$$

for $2 j+k \leq m$.

Proof. By Lemma 5.3 (iii) we have

$$
\llbracket \partial_{x^{\prime}} \sigma_{*}(t) \rrbracket_{m} \leq \llbracket \partial_{x^{\prime}} \sigma_{*}(t) \rrbracket_{m-1}+\llbracket \partial_{t} \sigma_{*}(t) \rrbracket_{m-2} \leq\left\|\left|D \sigma_{*}(t) \|\right|_{m-1} .\right.
$$

We use this estimate and others that come from properties of $\mathscr{Q}(t)$ and $\mathscr{P}(t)$, e.g.,

$$
\left\|\partial_{x^{\prime}}^{k} \sigma_{1}\right\|_{2} \leq\left\|\partial_{x^{\prime}} \sigma_{1}\right\|_{2}, k=1, \ldots,
$$

and

$$
\llbracket \nabla u_{1} \rrbracket_{m} \leq C \llbracket u_{1} \rrbracket_{m}
$$

together with Lemma 8.3 and Lemma 8.4 to obtain estimates (i)-(vi).

In the case of estimates (i)-(iii), we first use the following expansions and then apply above estimates:

$$
\begin{gathered}
\phi \operatorname{div} w=\sigma_{*} \phi^{(0)} w^{(0), 1} \partial_{x_{1}} \sigma_{*}+\sigma_{*} \phi^{(0)} \operatorname{div} w_{*}+\phi_{*} w^{(0), 1} \partial_{x_{1}} \sigma_{*}+\phi_{*} \operatorname{div} w_{*}, \\
w \cdot \nabla\left(\sigma_{*} \phi^{(0)}+\phi_{1}\right)= \\
\sigma_{*} \phi^{(0)} w^{(0), 1} \partial_{x_{1}} \sigma_{*}+w_{*}^{\prime} \cdot \nabla^{\prime} \sigma_{*} \phi^{(0)}+w_{*}^{n} \sigma_{*} \partial_{x_{n}} \phi^{(0)} \\
+\sigma_{*} w^{(0), 1} \partial_{x_{1}} \phi_{1}+w_{*} \cdot \nabla \phi_{1}, \\
w \cdot \nabla \phi_{\infty}=\sigma_{*} w^{(0), 1} \partial_{x_{1}} \phi_{\infty}+w_{*} \cdot \nabla \phi_{\infty} .
\end{gathered}
$$

This concludes the proof.

Second, we consider $\widetilde{Q} \boldsymbol{F}={ }^{T}(0, \boldsymbol{f})$. Recall that $\widetilde{Q} \boldsymbol{F}$ is written in the form

$$
\widetilde{Q} \boldsymbol{F}=\widetilde{\boldsymbol{F}}_{0}+\widetilde{\boldsymbol{F}}_{1}+\widetilde{\boldsymbol{F}}_{2}+\widetilde{\boldsymbol{F}}_{3} .
$$

Here, $\widetilde{\boldsymbol{F}}_{l}={ }^{T}\left(0, \boldsymbol{f}_{l}\right), l=0,1,2,3$, with

$$
\begin{gathered}
\boldsymbol{f}_{0}=-w \cdot \nabla w-f_{1}\left(\rho_{p}, \phi\right) \Delta^{\prime} \sigma_{*} w^{(0), 1} \boldsymbol{e}_{1}-f_{2}\left(\rho_{p}, \phi\right) \nabla\left(\partial_{x_{1}} \sigma_{*} w^{(0), 1}\right) \\
+\boldsymbol{f}_{01}\left(x_{n}, t, \phi\right) \phi \sigma_{*}+\boldsymbol{f}_{02}\left(x_{n}, \phi\right) \phi \nabla^{\prime} \sigma_{*}+\boldsymbol{f}_{03}\left(x_{n}, t, \phi\right) \phi \phi_{*}, \\
\boldsymbol{f}_{1}=-f_{1}\left(\rho_{p}, \phi\right) \Delta w_{*}=-\operatorname{div}\left(f_{1}\left(\rho_{p}, \phi\right) \nabla w_{*}\right)+{ }^{T}\left(\nabla w_{*}\right) \nabla\left(f_{1}\left(\rho_{p}, \phi\right)\right), \\
\boldsymbol{f}_{2}=-f_{2}\left(\rho_{p}, \phi\right) \nabla \operatorname{div} w_{*}=-\nabla\left(f_{2}\left(\rho_{p}, \phi\right) \operatorname{div} w_{*}\right)+\left(\operatorname{div} w_{*}\right) \nabla\left(f_{2}\left(\rho_{p}, \phi\right)\right), \\
\boldsymbol{f}_{3}=-f_{3}\left(x_{n}, \phi\right) \phi \nabla \phi_{*} .
\end{gathered}
$$

Here, $\nabla w_{*}$ denotes the $n \times n$ matrix $\left(\partial_{x_{i}} w_{*}^{j}\right) ; f_{1}=\frac{\nu \phi}{\rho_{p}\left(\gamma^{2} \rho_{p}+\phi\right)} ; f_{2}=\frac{\widetilde{\nu} \phi}{\rho_{p}\left(\gamma^{2} \rho_{p}+\phi\right)} ;$ and $\boldsymbol{f}_{0 l}\left(x_{n}, t, \phi\right), l=1,2,3$ and $f_{3}\left(x_{n}, \phi\right)$ are smooth functions of $x_{n}, t$ and $\phi$.

Proposition 8.6 Let $u(t)$ be solution of $(4.1)$ in $Z^{m}(\tau)$ and assume that $\sup _{0 \leq z \leq t} \llbracket u(z) \rrbracket_{m} \leq \varepsilon_{5}$ and $M(t) \leq 1$ for all $t \in[0, \tau]$. The following estimates hold for all $t \in[0, \tau]$ with $C>0$ independent of $\tau$.

$$
\llbracket \widetilde{Q} \boldsymbol{F}(t) \rrbracket_{m-2} \leq C(1+t)^{-\frac{2 n-1}{4}} M(t)^{2},
$$

(ii)

$$
\llbracket \boldsymbol{f}_{0}(t) \rrbracket_{m} \leq C\left\{(1+t)^{-\frac{2 n-1}{4}} M(t)^{2}+(1+t)^{-\frac{n-1}{4}} M(t)\left\|\left|D w_{\infty}(t) \|\right|_{m}\right\},\right.
$$


(iii)

$$
\sum_{l=1}^{3} \llbracket \boldsymbol{f}_{l}(t) \rrbracket_{m-1} \leq C\left\{(1+t)^{-\frac{n}{2}} M(t)^{2}+(1+t)^{-\frac{n-1}{4}} M(t)\left\|\left|D w_{\infty}(t) \|\right|_{m}\right\},\right.
$$

(iv)

$$
\sum_{l=1}^{3}\left\|T_{j, k} \boldsymbol{f}_{l}\right\|_{H^{-1}} \leq C\left\{(1+t)^{-\frac{n}{2}} M(t)^{2}+(1+t)^{-\frac{n-1}{4}} M(t)\left\|D w_{\infty}(t)\right\| \|_{m}\right\},
$$

for $2 j+k=m$. Here, we regard $T_{j, k} \boldsymbol{f}_{l}$ with $2 j+k=m$ as an element in $H^{-1}$ by $\left(T_{j, k} \boldsymbol{f}_{l}\right)[v]=$ $\left\langle T_{j, k} \boldsymbol{f}_{l}, v\right\rangle_{-1}$ for $v \in H_{0}^{1}$,

Proof. Since $\llbracket u(t) \rrbracket_{m} \leq \varepsilon_{5}$ we see that $\widetilde{Q} \boldsymbol{F}(t)$ is smooth. Estimates (i)-(iii) can be obtained in similar manner to the proof of Proposition 8.5 and we omit the proof.

Let us prove estimate (iv). Let $2 j+k=m$ and let $v \in H_{0}^{1}$. If $k \geq 1$ then we see from (iii) that

$$
\begin{gathered}
\left|\left\langle T_{j, k} \boldsymbol{f}_{l}, v\right\rangle_{-1}\right|=\left|-\left(T_{j, k-1} \boldsymbol{f}_{l}, \partial_{x^{\prime}} v\right)\right| \leq\left\|T_{j, k-1} \boldsymbol{f}_{l}\right\|_{2}\left\|\partial_{x^{\prime}} v\right\|_{v} \\
\leq C\left\{(1+t)^{-\frac{n}{2}} M(t)^{2}+(1+t)^{-\frac{n-1}{4}} M(t)\left\|\mid D w_{\infty}(t)\right\| \|_{m}\right\}\|v\|_{H_{0}^{1}} .
\end{gathered}
$$

We thus conclude that

$$
\left\|T_{j, k} \boldsymbol{f}_{l}\right\|_{H^{-1}} \leq C\left\{(1+t)^{-\frac{n}{2}} M(t)^{2}+(1+t)^{-\frac{n-1}{4}} M(t)\left\|\mid D w_{\infty}(t)\right\| \|_{m}\right\},
$$

in the case $2 j+k=m, k \geq 1$ and $l=1,2,3$.

If $k=0$, i.e., $m=2 j$, we write $\left\langle\partial_{t}^{j} \boldsymbol{f}_{1}, v\right\rangle_{-1}$ as

$$
\begin{gathered}
\left\langle\partial_{t}^{j} \boldsymbol{f}_{1}, v\right\rangle_{-1}=\left(\partial_{t}^{j}\left(f_{1}\left(\rho_{p}, \phi\right) \nabla w_{*}\right), \nabla v\right)+\left(\partial_{t}^{j}\left({ }^{T}\left(\nabla w_{*}\right) \partial_{\rho_{p}} f_{1}\left(\rho_{p}, \phi\right) \nabla \rho_{p}\right), v\right) \\
+\left(\left[\partial_{t}^{j},{ }^{T}\left(\nabla w_{*}\right) \partial_{\phi} f_{1}\left(\rho_{p}, \phi\right)\right] \nabla \phi, v\right)-\left({ }^{T}\left(\nabla w_{*}\right) \partial_{\phi} f_{1}\left(\rho_{p}, \phi\right) \partial_{t}^{j} \phi, \operatorname{div} v\right) \\
-\left({ }^{T}\left(\nabla^{2} w_{*}\right) \partial_{\phi} f_{1}\left(\rho_{p}, \phi\right) \partial_{t}^{j} \phi, v\right)-\left({ }^{T}\left(\nabla w_{*}\right) \nabla_{\rho_{p}, \phi} \partial_{\phi} f_{1}\left(\rho_{p}, \phi\right)\left(\nabla \rho_{p}+\nabla \phi\right) \partial_{t}^{j} \phi, v\right) \equiv \sum_{i=1}^{6} I_{i} .
\end{gathered}
$$

As for $I_{1}$, we have

$$
\left|I_{1}\right| \leq\left\|\partial_{t}^{j}\left(f_{1}\left(\rho_{p}, \phi\right) \nabla w_{*}\right)\right\|_{2}\|\nabla v\|_{2} .
$$

As in the proof of Proposition 8.5 (i) one can estimate $\left\|\partial_{t}^{j}\left(f_{1}\left(\rho_{p}, \phi\right) \nabla w_{*}\right)\right\|_{2}$ to obtain

$$
\left|I_{1}\right| \leq C\left\{(1+t)^{-\frac{2 n+1}{4}} M(t)^{2}+(1+t)^{-\frac{n}{4}} M(t)\left\|\left|D w_{\infty}(t) \|\right|_{m}\right\}\|v\|_{H_{0}^{1}} .\right.
$$

Similarly, we have

$$
\left|I_{2}\right| \leq C\left\{(1+t)^{-\frac{2 n+1}{4}} M(t)^{2}+(1+t)^{-\frac{n}{4}} M(t)\left\|\left|D w_{\infty}(t) \|\right|_{m}\right\}\|v\|_{H_{0}^{1}} .\right.
$$

Next, we consider $I_{3}$ which we write as follows:

$$
I_{3}=\left(\partial_{\phi} f_{1}\left(\rho_{p}, \phi\right)\left[\partial_{t}^{j},{ }^{T}\left(\nabla w_{*}\right)\right] \nabla \phi, v\right)+\left(\left[\partial_{t}^{j}, \partial_{\phi} f_{1}\left(\rho_{p}, \phi\right)\right]\left({ }^{T}\left(\nabla w_{*}\right) \nabla \phi\right), v\right) \equiv J_{1}+J_{2} .
$$

First, we treat $J_{1}$, we have

$$
\left|\left[\partial_{t}^{j},{ }^{T} \nabla w_{*}\right] \nabla \phi\right| \leq C \sum_{l=0}^{j-1}\left|\partial_{t}^{l} \nabla \phi\right|\left|\partial_{t}^{j-l} \partial_{x} w_{*}\right|
$$

Since

$$
\frac{1}{2}-\frac{m-1-2 l}{n}+\frac{1}{2}-\frac{m-2(j-l)}{n}=1-\frac{m-1}{n}<1,
$$

we can find $p_{1 l}, p_{2 l} \geq 2$ satisfying

$$
\frac{1}{p_{1 l}}>\frac{1}{2}-\frac{m-1-2 l}{n}, \frac{1}{p_{2 l}}>\frac{1}{2}-\frac{m-2(j-l)}{n}, \frac{1}{2} \leq \frac{1}{p_{1 l}}+\frac{1}{p_{2 l}}<1 .
$$


Now, we take number $p_{3 l} \geq 2$ satisfying $\frac{1}{p_{3 l}}=1-\left(\frac{1}{p_{1 l}}+\frac{1}{p_{2 l}}\right)>0$. It then follows from Lemma 8.2 (ii) that

$$
\begin{gathered}
\left|\left(\partial_{\phi} f_{1}\left(\rho_{p}, \phi\right)\left[\partial_{t}^{j},{ }^{T}\left(\nabla w_{*}\right)\right] \nabla \phi, v\right)\right| \leq C \sum_{l=0}^{j-1}\left\|\partial_{t}^{l} \partial_{x} \phi\right\|_{p_{1 l}}\left\|\partial_{t}^{j-l} \partial_{x} w_{*}\right\|_{p_{2 l}}\|v\|_{p_{3 l}} \\
\leq C \sum_{l=0}^{j-1}\left\|\partial_{t}^{l} \partial_{x} \phi\right\|_{H^{m-1-2 l}}\left\|\partial_{t}^{j-l} \partial_{x} w_{*}\right\|_{H^{m-2(j-l)}}\|v\|_{H_{0}^{1}} \leq C \llbracket \phi \rrbracket_{m}\left\{\llbracket \partial_{x} w_{1} \rrbracket_{m}+\left\|D w_{\infty}\right\| \|_{m}\right\}\|v\|_{H_{0}^{1}}
\end{gathered}
$$

Using Lemma 8.4 we conclude that

$$
\left|J_{1}\right| \leq C\left\{(1+t)^{-\frac{n}{2}} M(t)^{2}+(1+t)^{-\frac{n-1}{4}} M(t)\left\|\mid D w_{\infty}(t)\right\| \|_{m}\right\}\|v\|_{H_{0}^{1}} .
$$

Second, we estimate $J_{2}$. By Lemma 8.3 (i) we have for $m_{k}=m-1-2 k$ and $m_{l}=m-1-2 l$ that

$$
\begin{gathered}
\left\|\left[\partial_{t}^{j}, \partial_{\phi} f_{1}\left(\rho_{p}, \phi\right)\right]\left({ }^{T}\left(\nabla w_{*}\right) \nabla \phi\right)\right\|_{2} \leq C \sum_{k+1+l=j}\left\|\partial_{t}^{k}\left(\partial_{\phi}^{2} f_{1}\left(\rho_{p}, \phi\right) \partial_{t} \phi\right)\right\|_{m_{k}}\left\|\partial_{t}^{l}\left(T^{T}\left(\nabla w_{*}\right) \nabla \phi\right)\right\|_{m_{l}} \\
\leq C \llbracket \partial_{t} \phi \rrbracket_{m-1} \rrbracket^{T}\left(\nabla w_{*}\right) \nabla \phi \rrbracket_{m-1} .
\end{gathered}
$$

Therefore, we obtain

$$
\left|J_{2}\right| \leq C \llbracket \partial_{t} \phi \rrbracket_{m-1} \llbracket^{T}\left(\nabla w_{*}\right) \nabla \phi \rrbracket_{m-1}\|v\|_{2},
$$

By Lemma 8.3 (ii) we get

$$
\begin{gathered}
\llbracket^{T}\left(\nabla w_{*}\right) \nabla \phi \rrbracket_{m-1} \leq C\left\{\left\|\nabla w_{*}\right\|_{\infty} \llbracket \partial_{x} \phi \rrbracket_{m-1}+\left\|\left|D \nabla w_{*} \|\right|_{m-2} \llbracket \nabla \phi \rrbracket_{m-1}\right\}\right. \\
\leq C\left\{\left\|\partial_{x} w_{1}\right\|_{H^{m}}+\left\|\mid D w_{\infty}\right\| \|_{m}\right\} \llbracket \partial_{x} \phi \rrbracket_{m-1} \leq C\left\{(1+t)^{-\frac{n}{2}} M(t)^{2}+(1+t)^{-\frac{n-1}{4}} M(t)\left\|D w_{\infty}(t)\right\| \|_{m}\right\} .
\end{gathered}
$$

As for $\llbracket \partial_{t} \phi \rrbracket_{m-1}$, we see from (6.3) and Proposition 7.9 that

$$
\begin{gathered}
\llbracket \partial_{t} \phi \rrbracket_{m-1} \leq C\left\{\llbracket \partial_{t} \sigma_{*} \rrbracket_{m-1}+\llbracket \partial_{t} \phi_{*} \rrbracket_{m-1}\right\} \leq C\left\{\llbracket \Lambda \sigma_{1}(t) \rrbracket_{m-1}+\llbracket \mathscr{P}(t) \boldsymbol{F}(t) \rrbracket_{m-1}\right. \\
\left.+\llbracket \partial_{x^{\prime}} \phi_{\infty} \rrbracket_{m-1}+\llbracket \partial_{x} w_{\infty} \rrbracket_{m-1}+\llbracket \partial_{x^{\prime}} \sigma_{\infty} \rrbracket_{m-1}+\llbracket\left[Q_{0}\left(P_{\infty}^{(0)} \boldsymbol{F}\right)\right]_{\infty} \rrbracket_{m-1}+\llbracket Q_{0} P_{\infty} \boldsymbol{F} \rrbracket_{m-1}\right\} .
\end{gathered}
$$

Using

$$
P_{\infty} \boldsymbol{F}=\boldsymbol{F}-\left[Q_{0} \boldsymbol{F}\right]_{\infty} u^{(0)}-\left\{\mathbb{P} \boldsymbol{F}-\left[Q_{0} \mathbb{P} \boldsymbol{F}\right]_{\infty} u^{(0)}\right\}
$$

and

$$
\left[Q_{0} P_{\infty}^{(0)} \boldsymbol{F}\right]_{\infty}=\left[Q_{0} \boldsymbol{F}\right]_{\infty}-\left[Q_{0} \mathbb{P} \boldsymbol{F}\right]_{\infty}
$$

together with Lemma 8.4 we get

$$
\llbracket \partial_{t} \phi \rrbracket_{m-1} \leq C\left\{(1+t)^{-\frac{n+1}{4}} M(t)+\llbracket \mathscr{P}(t) \boldsymbol{F}(t) \rrbracket_{m-1}+\llbracket Q_{0} \boldsymbol{F} \rrbracket_{m-1}\right\} .
$$

Since $2 j=m$, we have $\left[\frac{m-1}{2}\right]=\left[\frac{m-2}{2}\right]$, and hence, by properties of $\mathscr{P}(t)$,

$$
\llbracket \mathscr{P}(t) \boldsymbol{F}(t) \rrbracket_{m-1} \leq C \llbracket \boldsymbol{F}(t) \rrbracket_{m-2} .
$$

It then follows from Propositions 8.5 (i)-(iii) and 8.6 (i) that

$$
\llbracket \boldsymbol{F}(t) \rrbracket_{m-2}+\llbracket Q_{0} \boldsymbol{F}(t) \rrbracket_{m-1} \leq 2 \llbracket Q_{0} \boldsymbol{F}(t) \rrbracket_{m-1}+\llbracket \widetilde{Q} \boldsymbol{F}(t) \rrbracket_{m-2} \leq C(1+t)^{-\frac{2 n-1}{4}} M(t)^{2},
$$

which implies

$$
\llbracket \partial_{t} \phi \rrbracket_{m-1} \leq C(1+t)^{-\frac{n+1}{4}} M(t)
$$

We thus conclude

$$
\left|J_{2}\right| \leq C\left\{(1+t)^{-\frac{3 n+1}{4}} M(t)^{3}+(1+t)^{-\frac{n}{2}} M(t)^{2}\left\|\mid D w_{\infty}(t)\right\| \|_{m}\right\}\|v\|_{2} .
$$

Consequently, 


$$
\left|I_{3}\right| \leq C\left\{(1+t)^{-\frac{n}{2}} M(t)^{2}+(1+t)^{-\frac{n-1}{4}} M(t)\left\|\left|D w_{\infty}(t) \|\right|_{m}\right\}\|v\|_{H_{0}^{1}} .\right.
$$

Since

$$
\left|I_{4}\right| \leq\left|\left({ }^{T}\left(\nabla w_{*}\right) \partial_{\phi} f_{1}\left(\rho_{p}, \phi\right) \partial_{t}^{j} \phi, \operatorname{div} v\right)\right| \leq C\left\|\nabla w_{*}\right\|\left\|_{\infty}\right\| \partial_{t}^{j} \phi\left\|_{2}\right\| \nabla v \|_{2},
$$

and by Lemma $8.3(\mathrm{i})$,

$$
\begin{gathered}
\left|I_{5}\right| \leq\left|\left({ }^{T}\left(\nabla^{2} w_{*}\right) \partial_{\phi} f_{1}\left(\rho_{p}, \phi\right) \partial_{t}^{j} \phi, v\right)\right| \leq C\left\|\partial_{t}^{j} \phi\right\|_{2}\left\|v \nabla^{2} w_{*}\right\|_{2} \leq C\left\|\partial_{t}^{j} \phi\right\|_{2}\left\|\nabla w_{*}\right\|_{H^{m}}\|v\|_{H^{1}}, \\
\left.\left|I_{6}\right| \leq \mid{ }^{T}\left(\nabla w_{*}\right) \nabla_{\rho_{p}, \phi} \partial_{\phi} f_{1}\left(\rho_{p}, \phi\right)\left(\nabla \rho_{p}+\nabla \phi\right) \partial_{t}^{j} \phi, v\right) \mid \leq C\left\|\nabla w_{*}\right\|_{\infty}\left\|\partial_{t}^{j} \phi\right\|_{2}\|v\|_{H^{1}}\left\|\rho_{p}+\phi\right\|_{H^{m}},
\end{gathered}
$$

we obtain by Lemmas 8.2 (ii) and 8.4,

$$
\left|I_{4}\right|+\left|I_{5}\right|+\left|I_{6}\right| \leq C\left\{(1+t)^{-\frac{n+1}{2}} M(t)^{2}+(1+t)^{-\frac{n+1}{4}} M(t)\left\|\left|D w_{\infty}(t) \|\right|_{m}\right\}\|v\|_{H_{0}^{1}} .\right.
$$

Therefore, we arrive at

$$
\left|\left\langle\partial_{t}^{j} \boldsymbol{f}_{1}, v\right\rangle_{-1}\right| \leq C\left\{(1+t)^{-\frac{n}{2}} M(t)^{2}+(1+t)^{-\frac{n-1}{4}} M(t)\left\|\mid D w_{\infty}(t)\right\| \|_{m}\right\}\|v\|_{H_{0}^{1}} .
$$

This gives

$$
\left\|\partial_{t}^{j} \boldsymbol{f}_{1}\right\|_{H^{-1}} \leq C\left\{(1+t)^{-\frac{n}{2}} M(t)^{2}+(1+t)^{-\frac{n-1}{4}} M(t)\left\|\mid D w_{\infty}(t)\right\| \|_{m}\right\} .
$$

Clearly, one can get the same estimate for $\left\|\partial_{t}^{j} \boldsymbol{f}_{2}\right\|_{H^{-1}}$. Concerning $\left\|\partial_{t}^{j} \boldsymbol{f}_{3}\right\|_{H^{-1}}$, one can write it as

$$
\partial_{t}^{j} \boldsymbol{f}_{3}=-\left[\partial_{t}^{j}, f_{3}\left(x_{n}, \phi\right) \phi\right] \nabla \phi_{*}-\nabla\left(f_{3}\left(x_{n}, \phi\right) \phi \partial_{t}^{j} \phi_{*}\right)+\nabla\left(f_{3}\left(x_{n}, \phi\right) \phi\right) \partial_{t}^{j} \phi_{*},
$$

and thus the desired estimate is obtained analogously to the one for $\left\|\partial_{t}^{j} \boldsymbol{f}_{1}\right\|_{H^{-1}}$. This completes the proof.

Proof of Proposition 8.1. Since $\llbracket \widetilde{Q} P_{\infty} \boldsymbol{F} \rrbracket_{m-2} \leq C \llbracket \boldsymbol{F} \rrbracket_{m-2}$, assertion (i) follows from Propositions 8.5 (i)-(iii) and 8.6 (i).

Let us consider $\widetilde{R}(t)$. We know that there holds

$$
\widetilde{R}(t) \leq C\left(\sum_{j=1}^{8} R^{(j)}(t)+\sum_{p=1}^{m-1} R_{p}^{(9)}\right) .
$$

Let us first show some basic estimates coming from Propositions 8.5, 8.6 and properties of $P(t)$ :

$$
\begin{gathered}
\llbracket Q_{0} \boldsymbol{F} \rrbracket_{m-1} \leq C(1+t)^{-\frac{2 n+1}{4}} M(t)^{2}, \\
\llbracket \widetilde{Q} \boldsymbol{F} \rrbracket_{m-1} \leq C\left\{(1+t)^{-\frac{2 n-1}{4}} M(t)^{2}+\left.(1+t)^{-\frac{n-1}{4}} M(t)\left\|D w_{\infty}(t)\right\|\right|_{m}\right\}, \\
\llbracket \mathbb{P} \boldsymbol{F} \rrbracket_{m-1} \leq C \llbracket \boldsymbol{F} \rrbracket_{m-1},
\end{gathered}
$$

Moreover, there holds

$$
\left[Q_{0} T_{j, k} \boldsymbol{F}\right]_{\infty}=-\left[\operatorname{div} T_{j, k}(\phi w)\right]_{\infty}=-\left[\operatorname{div}^{\prime} T_{j, k}\left(\phi w^{\prime}\right)\right]_{\infty},
$$

since $w \in H_{0}^{1}$.

Let us begin with $R_{j, k}^{(1)}$. We write

$$
\begin{gathered}
R_{j, k}^{(1)}=\frac{\alpha_{0}}{\gamma^{2}}\left(\left[Q_{0} T_{j, k} \boldsymbol{F}\right]_{\infty}, T_{j, k} \sigma_{\infty}\right)-\frac{\alpha_{0}}{\gamma^{2}}\left(\left[Q_{0} T_{j, k}(\mathbb{P} \boldsymbol{F})\right]_{\infty}, T_{j, k} \sigma_{\infty}\right)+\widetilde{R}_{j, k}^{(1)}-\left\langle T_{j, k}\left(\left[Q_{0} \boldsymbol{F}\right]_{\infty} u^{(0)}\right), T_{j, k} u_{\infty}\right\rangle_{\Omega} \\
-\left\langle T_{j, k}(\mathbb{P} \boldsymbol{F}), T_{j, k} u_{\infty}\right\rangle_{\Omega}+\left\langle T_{j, k}\left(\left[Q_{0}(\mathbb{P} \boldsymbol{F})\right]_{\infty} u^{(0)}\right), T_{j, k} u_{\infty}\right\rangle_{\Omega}=\sum_{l=1}^{6} I_{l}
\end{gathered}
$$

Here,

$$
I_{3}=\widetilde{R}_{j, k}^{(1)}=\left\langle T_{j, k} \boldsymbol{F}, T_{j, k} u_{\infty}\right\rangle_{\Omega},
$$


when $2 j+k \leq m-1$, and

$$
\begin{gathered}
I_{3}=\widetilde{R}_{j, k}^{(1)}=-\left(T_{j, k}(\phi \operatorname{div} w), T_{j, k} \phi_{\infty} \frac{P^{\prime}\left(\rho_{p}\right)}{\gamma^{4} \rho_{p}}\right)+\frac{1}{2}\left(\operatorname{div}\left(\frac{P^{\prime}\left(\rho_{p}\right)}{\gamma^{4} \rho_{p}} w\right),\left|T_{j, k} \phi_{\infty}\right|^{2}\right) \\
-\left(w \nabla T_{j k}\left(\sigma_{*} \phi^{(0)}+\phi_{1}\right), T_{j k} \phi_{\infty} \frac{P^{\prime}\left(\rho_{p}\right)}{\gamma^{4} \rho_{p}}\right)-\left(\left[T_{j, k}, w\right] \nabla \phi, T_{j, k} \phi_{\infty} \frac{P^{\prime}\left(\rho_{p}\right)}{\gamma^{4} \rho_{p}}\right) . \\
+\left(T_{j, k} \boldsymbol{f}_{0}, T_{j, k} w_{\infty} \rho_{p}\right)+\sum_{l=1}^{3}\left\langle T_{j, k} \boldsymbol{f}_{l}, T_{j, k} w_{\infty} \rho_{p}\right\rangle_{-1},
\end{gathered}
$$

when $2 j+k=m$.

We first consider $I_{3}$. If $2 j+k \leq m-1$, then by applying (8.1) and (8.2), we have

$$
\sum_{2 j+k \leq m-1}\left|\left\langle T_{j, k} \boldsymbol{F}, T_{j, k} u_{\infty}\right\rangle_{\Omega}\right| \leq C \llbracket \boldsymbol{F} \rrbracket_{m-1} \llbracket u_{\infty} \rrbracket_{m-1} \leq C\left\{(1+t)^{-\frac{3 n}{4}} M(t)^{3}+(1+t)^{-\frac{n}{4}} M(t) D_{\infty}(t)\right\} .
$$

Here, we used relation $a^{2} b \leq \frac{1}{2}\left(a^{3}+a b^{2}\right)$ and Lemma 8.4 (ii).

In the case $2 j+k=m$, we use Lemma 8.4 to calculate

$$
\left|\left(w \nabla T_{j k}\left(\sigma_{*} \phi^{(0)}+\phi_{1}\right), T_{j k} \phi_{\infty} \frac{P^{\prime}\left(\rho_{p}\right)}{\gamma^{4} \rho_{p}}\right)\right| \leq C\|w\|_{\infty}\left(\llbracket \sigma_{*} \rrbracket_{m}+\llbracket \phi_{1} \rrbracket_{m}\right) \llbracket \phi_{\infty} \rrbracket_{m} \leq C(1+t)^{-n} M(t)^{3} .
$$

From above estimate and Propositions 8.5 (i), (iv), (v) and 8.6 (ii), (iv) we see that

$$
\sum_{2 j+k \leq m}\left|I_{3}\right| \leq C\left\{(1+t)^{-\frac{3 n}{4}} M(t)^{3}+(1+t)^{\frac{n-1}{4}} M(t) D_{\infty}(t)\right\} .
$$

We next consider $I_{5}$. If $2 j+k \leq m-1$, then by (8.3) we see that

$$
\sum_{2 j+k \leq m-1}\left|\left\langle T_{j, k}(\mathbb{P} \boldsymbol{F}), T_{j, k} u_{\infty}\right\rangle_{\Omega}\right| \leq C \llbracket \mathbb{P} \boldsymbol{F} \rrbracket_{m-1} \llbracket u_{\infty} \rrbracket_{m-1} \leq C \llbracket \boldsymbol{F} \rrbracket_{m-1} \llbracket u_{\infty} \rrbracket_{m-1} .
$$

If $2 j+k=m$ and $k \geq 1$, then from properties of $P(t)$ we obtain

$$
\sum_{2 j+k=m \mid k \geq 1}\left|\left\langle T_{j, k}(\mathbb{P} \boldsymbol{F}), T_{j, k} u_{\infty}\right\rangle_{\Omega}\right| \leq \sum_{2 j+k=m \mid k \geq 1} C\left\|T_{j, k-1}(\mathbb{P} \boldsymbol{F})\right\|_{2}\left\|T_{j, k} u_{\infty}\right\|_{2} \leq C \llbracket \boldsymbol{F} \rrbracket_{m-1} \llbracket u_{\infty} \rrbracket_{m} .
$$

In the case $2 j=m$, we write

$$
\left|\left\langle\partial_{t}^{j}(\mathbb{P} \boldsymbol{F}), \partial_{t}^{j} u_{\infty}\right\rangle_{\Omega}\right| \leq C\left|\left\langle\left[\partial_{t}^{j}, \mathbb{P}\right] \boldsymbol{F}, \partial_{t}^{j} u_{\infty}\right\rangle_{\Omega}\right|+\left|\left\langle\mathbb{P} \partial_{t}^{j} \boldsymbol{F}, \partial_{t}^{j} u_{\infty}\right\rangle_{\Omega}\right| \leq C \llbracket \boldsymbol{F} \rrbracket_{m-2} \llbracket u_{\infty} \rrbracket_{m}+\left|\left\langle\mathbb{P} \partial_{t}^{j} \boldsymbol{F}, \partial_{t}^{j} u_{\infty}\right\rangle_{\Omega}\right| .
$$

To estimate $\left\langle\mathbb{P} \partial_{t}^{j} \boldsymbol{F}, \partial_{t}^{j} u_{\infty}\right\rangle_{\Omega}$, we write it as

$$
\left\langle\mathbb{P} \partial_{t}^{j} \boldsymbol{F}, \partial_{t}^{j} u_{\infty}\right\rangle_{\Omega}=\left\langle\partial_{t}^{j} \boldsymbol{F}, \mathbb{P}^{*} \partial_{t}^{j} u_{\infty}\right\rangle_{\Omega} .
$$

Using integration by parts, we have

$$
\left\langle\partial_{t}^{j} Q_{0} \boldsymbol{F}, P^{*} \partial_{t}^{j} u_{\infty}\right\rangle_{\Omega}=\left(\partial_{t}^{j}(\phi w), \nabla\left(Q_{0}\left(\mathbb{P}^{*} \partial_{t}^{j} u_{\infty}\right) \frac{P^{\prime}\left(\rho_{p}\right)}{\gamma^{4} \rho_{p}}\right)\right) .
$$

Since

$$
\left\|\nabla\left(Q_{0}\left(\mathbb{P}^{*} \partial_{t}^{j} u_{\infty}\right) \frac{P^{\prime}\left(\rho_{p}\right)}{\gamma^{4} \rho_{p}}\right)\right\|_{2} \leq C\left\|\partial_{t}^{j} u_{\infty}\right\|_{2}
$$

by properties of $\mathbb{P}^{*}$, we see from Proposition 8.5 (vi) that

$$
\left|\left\langle\partial_{t}^{j} Q_{0} \boldsymbol{F}, \mathbb{P}^{*} \partial_{t}^{j} u_{\infty}\right\rangle_{\Omega}\right| \leq C(1+t)^{-\frac{3 n}{4}} M(t)^{3} .
$$

Since $\widetilde{Q} \mathbb{P}^{*} \partial_{t}^{j} u_{\infty} \in H_{0}^{1}$ one can estimate $\left|\left\langle\partial_{t}^{j} \widetilde{Q} \boldsymbol{F}, \mathbb{P}^{*} \partial_{t}^{j} u_{\infty}\right\rangle_{\Omega}\right|$ using Proposition 8.6 (ii), (iv) to obtain

$$
\sum_{2 j=m}\left|\left\langle\partial_{t}^{j} \widetilde{Q} \boldsymbol{F}, \mathbb{P}^{*} \partial_{t}^{j} u_{\infty}\right\rangle_{\Omega}\right| \leq C\left\{(1+t)^{-\frac{3 n}{4}} M(t)^{3}+(1+t)^{\frac{n-1}{4}} M(t) D_{\infty}(t)\right\}
$$


Therefore, we have

$$
\sum_{2 j+k \leq m}\left|I_{5}\right| \leq C\left\{(1+t)^{-\frac{3 n}{4}} M(t)^{3}+(1+t)^{\frac{n-1}{4}} M(t) D_{\infty}(t)\right\} .
$$

As for $I_{4}$, from (8.4) we compute

$$
-\left\langle T_{j, k}\left(\left[Q_{0} \boldsymbol{F}\right]_{\infty} u^{(0)}\right), T_{j, k} u_{\infty}\right\rangle_{\Omega}=\sum_{i=0}^{j}\left(\begin{array}{c}
j \\
i
\end{array}\right)\left\langle\left[\operatorname{div}^{\prime} T_{i, k}\left(\phi w^{\prime}\right)\right]_{\infty} T_{j-i, 0} u^{(0)}, T_{j, k} u_{\infty}\right\rangle_{\Omega} .
$$

Since

$$
\left\|\left[\operatorname{div}^{\prime} T_{i, k}\left(\phi w^{\prime}\right)\right]_{\infty} T_{j-i, 0} u^{(0)}\right\|_{2} \leq C\left\|T_{i, k}\left(\phi w^{\prime}\right)\right\|_{2},
$$

we see using Proposition 8.5 (vi) that

$$
\sum_{2 j+k \leq m}\left|I_{4}\right| \leq C(1+t)^{-\frac{3 n}{4}} M(t)^{3} .
$$

As for $I_{1}$, using (8.4) and integration by parts we obtain

$$
\frac{\alpha_{0}}{\gamma^{2}}\left(\left[Q_{0} T_{j, k} \boldsymbol{F}\right]_{\infty}, T_{j, k} \sigma_{\infty}\right)=-\frac{\alpha_{0}}{\gamma^{2}}\left(\left[\operatorname{div}^{\prime} T_{j, k}\left(\phi w^{\prime}\right)\right]_{\infty}, T_{j, k} \sigma_{\infty}\right) \leq C\left\|T_{j, k}(\phi w)\right\|_{2}\left\|\nabla^{\prime} T_{j, k} \sigma_{\infty}\right\|_{2} .
$$

and thus by Proposition 8.5 (vi) and Lemma 8.4 (ii) we get

$$
\sum_{2 j+k \leq m}\left|I_{1}\right| \leq C(1+t)^{-\frac{3 n}{4}} M(t)^{3} .
$$

As for $I_{2}$, in the case $1 \leq 2 j+k \leq m$ we treat it analogously to $I_{5}$ to show

$$
\left|\frac{\alpha_{0}}{\gamma^{2}}\left(\left[Q_{0} T_{j, k}(\mathbb{P} \boldsymbol{F})\right]_{\infty}, T_{j, k} \sigma_{\infty}\right)\right| \leq C \llbracket \boldsymbol{F} \rrbracket_{m-1} \llbracket \partial_{x^{\prime}} \sigma_{\infty} \rrbracket_{m-1}+\left|\left(\left[Q_{0} \mathbb{P} \partial_{t}^{\left[\frac{m}{2}\right]} \boldsymbol{F}\right]_{\infty}, \partial_{t}^{\left[\frac{m}{2}\right]} \sigma_{\infty}\right)\right| .
$$

We further estimate

$$
\left|\left(\left[Q_{0} \mathbb{P} \partial_{t}^{\left[\frac{m}{2}\right]} \boldsymbol{F}\right]_{\infty}, \partial_{t}^{\left[\frac{m}{2}\right]} \sigma_{\infty}\right)\right| \leq\left\|\mathscr{P}\left(\partial_{t}^{\left[\frac{m}{2}\right]} \boldsymbol{F}\right)\right\|_{2}\left\|\partial_{t}^{\left[\frac{m}{2}\right]} \sigma_{\infty}\right\|_{2}
$$

Using Plancherel theorem we have

$$
\left\|\mathscr{P}(t)\left(\partial_{t}^{\left[\frac{m}{2}\right]} \boldsymbol{F}(t)\right)\right\|_{2}=\left\|\widehat{\chi}_{1}\left\langle\partial_{t}^{\left[\frac{m}{2}\right]} \widehat{\boldsymbol{F}}(t), u_{\xi^{\prime}}^{*}(t)\right\rangle\right\|_{2}
$$

Therefore, using above relation, analogously to $I_{5}$, we estimate

$$
\begin{gathered}
\left\|\mathscr{P}(t)\left(\partial_{t}^{\left[\frac{m}{2}\right]} \boldsymbol{F}(t)\right)\right\|_{2} \leq\left\|\mathscr{P}(t)\left(Q_{0} \partial_{t}^{\left[\frac{m}{2}\right]} \boldsymbol{F}(t)\right)\right\|_{2}+\left\|\mathscr{P}(t)\left(\widetilde{Q} \partial_{t}^{\left[\frac{m}{2}\right]} \boldsymbol{F}(t)\right)\right\|_{2} \\
\leq C\left\{\left\|\partial_{t}^{\left[\frac{m}{2}\right]}(\phi w)\right\|_{2}+\llbracket \boldsymbol{f}_{0} \rrbracket_{m}+\sum_{l=1}^{3}\left\|\partial_{t}^{\left[\frac{m}{2}\right]} \boldsymbol{f}_{l}\right\|_{H^{-1}}\right.
\end{gathered}
$$

In the case $j=k=0$ we see from Lemma 6.1 (ii) and properties of $\mathbb{P}(t)$,

$$
\left|\frac{\alpha_{0}}{\gamma^{2}}\left(\left[Q_{0}(\mathbb{P} \boldsymbol{F})\right]_{\infty}, \sigma_{\infty}\right)\right| \leq C\left\|\partial_{x^{\prime}} \sigma_{\infty}\right\|_{2}\left(\left\|\phi w^{\prime}\right\|_{2}+\|\boldsymbol{F}\|_{2}\right) \leq(1+t)^{-\frac{3 n}{4}} M(t)^{3} .
$$

Therefore, using Propositions 8.5 (vi) and 8.6 (ii), (iv) we obtain

$$
\sum_{2 j+k \leq m}\left|I_{2}\right| \leq C\left\{(1+t)^{-\frac{3 n}{4}} M(t)^{3}+(1+t)^{-\frac{n-1}{4}} M(t) D_{\infty}(t)\right\}
$$

As for $I_{6}$, it can be treated in a way analogous to $I_{5}$ in the case $1 \leq 2 j+k$, thus we get

$$
\sum_{2 j+k \leq m}\left|\left\langle T_{j, k}\left(\left[Q_{0}(\mathbb{P} \boldsymbol{F})\right]_{\infty} u^{(0)}\right), T_{j, k} u_{\infty}\right\rangle_{\Omega}\right| \leq C\left\{\llbracket \boldsymbol{F} \rrbracket_{m-1} \llbracket u_{\infty} \rrbracket_{m-1}+\left\|\mathscr{P}\left(\partial_{t}^{\left[\frac{m}{2}\right]} \boldsymbol{F}\right)\right\|_{2}\left\|\partial_{t}^{\left[\frac{m}{2}\right]} u_{\infty}\right\|_{2}\right\}
$$

and 


$$
\sum_{2 j+k \leq m}\left|I_{6}\right| \leq C\left\{(1+t)^{-\frac{3 n}{4}} M(t)^{3}+(1+t)^{\frac{n-1}{4}} M(t) D_{\infty}(t)\right\} .
$$

We thus conclude

$$
R^{(1)}(t) \leq C\left\{(1+t)^{-\frac{3 n}{4}} M(t)^{3}+(1+t)^{\frac{n-1}{4}} M(t) D_{\infty}(t)\right\} .
$$

It is straightforward to show that

$$
\begin{gathered}
\widetilde{R}(t) \leq C\left\{R^{(1)}(t)+\llbracket \boldsymbol{F} \rrbracket_{m-1}^{2}+\llbracket Q_{0}\left(P_{\infty}^{(0)} \boldsymbol{F}\right) \rrbracket_{m-1}^{2}+\llbracket P_{\infty} \boldsymbol{F} \rrbracket_{m-1}^{2}+\llbracket \phi \operatorname{div} w \rrbracket_{m}^{2}\right. \\
+\llbracket w \cdot \nabla\left(\sigma_{*} \phi^{(0)}+\phi_{1}\right) \rrbracket_{m}^{2}+\sum_{2 j+k \leq m}\left\|\left[\partial_{t}^{j} \partial_{x}^{k}, w\right] \cdot \nabla \phi_{\infty}\right\|_{2}^{2} \\
\quad+\sum_{2 j+k+l \leq m-1}\left|\left(\operatorname{div}\left(\frac{P^{\prime}\left(\rho_{p}\right)}{\gamma^{4} \rho_{p}} w\right),\left|T_{j, k} \partial_{x_{n}}^{l+1} \phi_{\infty}\right|^{2}\right)\right| \\
\left.+\sum_{2 j+k \leq m-1}\left|\left(Q_{0} T_{j, k}\left(P_{\infty}^{(0)} \boldsymbol{F}\right), T_{j, k} \sigma_{\infty}\right)\right|+\llbracket \widetilde{Q}\left(P_{\infty} \boldsymbol{F}\right) \rrbracket_{m-1} \llbracket \partial_{x^{\prime}} \sigma_{\infty} \rrbracket_{m-1}\right\} .
\end{gathered}
$$

From definition we have

$$
P_{\infty}^{(0)}(t)=I-\mathbb{P}(t)-P_{\infty, 3},
$$

which together with

$$
\left|\left(Q_{0} P_{\infty, 3} T_{j, k} \boldsymbol{F}, T_{j, k} \sigma_{\infty}\right)\right| \leq C\left\|T_{j k}(\phi w)\right\|_{2}\left\|\left|D \sigma_{\infty} \|\right|_{m-1} .\right.
$$

gives (analogously to previous computations)

$$
\sum_{2 j+k \leq m-1}\left|\left(Q_{0} T_{j, k}\left(P_{\infty}^{(0)} \boldsymbol{F}\right), T_{j, k} \sigma_{\infty}\right)\right| \leq C(1+t)^{-\frac{3 n}{4}} M(t)^{3} .
$$

Since

$$
\llbracket P_{\infty}^{(0)} \boldsymbol{F} \rrbracket_{m-1}+\llbracket P_{\infty} \boldsymbol{F} \rrbracket_{m-1} \leq C \llbracket \boldsymbol{F} \rrbracket_{m-1},
$$

using Propositions 8.5, 8.6 and Lemma 8.4 we obtain the desired estimate (ii) in Proposition 8.1. This completes the proof.

\section{Asymptotic behavior of $\sigma_{1}(t)$}

In this section we show the asymptotic behavior of solutions of (4.1). In the case $n=2$ we prove that it is described by a solution of a 1-dimensional viscous Burgers equation. In the case $n \geq 3$ we show that the asymptotic behavior is described by a linear heat equation, in fact, asymptotic leading term is the same as for the linearized problem.

In this section we assume that $\nu \geq \nu_{0}$ and $\gamma^{2} /(\nu+\widetilde{\nu}) \geq \gamma_{0}^{2}$. Let us note that $\sigma_{1}(t)$ is given by

$$
\sigma_{1}(t)=\mathscr{P}(t) u(t), t \geq 0
$$

where $u(t)$ is a global in time solution of (4.1). Existence of $u(t)$ was proved in Sections $3-8$.

First let us treat the case $n=2$.

Lemma 9.1 Let $n=2$ and $\sigma(t)$ is a solution of

$$
\begin{gathered}
\partial_{t} \sigma-\kappa_{1} \partial_{x_{1}}^{2} \sigma+\kappa_{0} \partial_{x_{1}} \sigma+\omega_{0} \partial_{x_{1}}\left(\sigma^{2}\right)=0 \\
\left.\sigma\right|_{t=0}=\sigma_{0}
\end{gathered}
$$

where $\kappa_{0} \in \mathbb{R}, \kappa_{1}>0$ are the numbers given in (4.6) and $\omega_{0}=\frac{1}{T} \int_{0}^{T}\left[\phi^{(0)} w^{(0), 1}(z)\right]-\left\langle\boldsymbol{F}_{1}(z), u^{*(1)}(z)\right\rangle d z$ and $\sigma_{0}=\left[Q_{0} u_{0}\right]=\left[\phi_{0}\right]$. Then we can write

$$
\sigma(t)=\mathscr{H}(t) \sigma_{0}-\omega_{0} \int_{0}^{t} \mathscr{H}(t-z) \partial_{x_{1}}\left(\sigma^{2}(z)\right) d z
$$


Theorem 9.2 Let $n=2$. For any $\delta>0$ there exists $\varepsilon_{7}>0$ such that if $\left\|u_{0}\right\|_{H^{m} \cap L^{1}} \leq \varepsilon_{7}$, then

$$
\left\|\sigma_{1}(t)-\sigma(t)\right\|_{2} \leq C(1+t)^{-\frac{3}{4}+\delta}\left\|u_{0}\right\|_{H^{m} \cap L^{1}}
$$

for $t \geq 0$.

(3.7) now follows from (5.13) and Theorem 9.2. To prove Theorem 9.2, we employ the following well-known decay properties of $\sigma(t)$.

Lemma 9.3 Let $n=2$ and $\sigma(t)$ is a solution of $(9.1)$ with $\left\|\sigma_{0}\right\|_{H^{1} \cap L^{1}} \ll 1$. Then

$$
\begin{gathered}
\left\|\partial_{x_{1}}^{k} \sigma(t)\right\|_{2} \leq C(1+t)^{-\frac{1}{4}-\frac{k}{2}}\left\|\sigma_{0}\right\|_{H^{1} \cap L^{1}} \quad(k=0,1), \\
\|\sigma(t)\|_{\infty} \leq C(1+t)^{-\frac{1}{2}}\left\|\sigma_{0}\right\|_{H^{1} \cap L^{1}}
\end{gathered}
$$

We introduce a quantity. Let $\sigma_{1}(t)$ and $\sigma(t)$ be solutions of (5.1) for $s=0$ and (9.1), respectively. We define $N(t)$ by

$$
N(t)=\sup _{0 \leq z \leq t}(1+z)^{\frac{3}{4}-\delta}\left\|\sigma_{1}(z)-\sigma(z)\right\|_{H^{1}}
$$

Theorem 9.2 would then follow if we could show that $N(t) \leq C\left\|u_{0}\right\|_{H^{m} \cap L^{1}}$.

Proof of Theorem 9.2. It is obvious that estimate holds for $0 \leq t<1$. Let us show that it holds for $t \geq 1$. Assume $t \geq 1$. From (5.1) we have that for $s=0$

$$
\sigma_{1}(t)=e^{t \Lambda} \mathscr{P}(0) u_{0}+\int_{0}^{t} e^{(t-z) \Lambda} \mathscr{P}(z) \boldsymbol{F}(z) d z .
$$

We next rewrite $e^{(t-z) \Lambda} \mathscr{P}(z) \boldsymbol{F}(z)$. By Lemma 6.1 (ii), we have

$$
\begin{gathered}
\mathscr{P}(z) \boldsymbol{F}(z)=-\partial_{x_{1}}\left[\phi w^{1}\right]_{1}+\partial_{x_{1}} \mathscr{P}^{(1)}(z) \boldsymbol{F}(z)+\partial_{x_{1}}^{2} \mathscr{P}^{(2)}(z) \boldsymbol{F}(z) \\
=-a_{11}(z) \partial_{x_{1}}\left(\sigma_{1}^{2}\right)-\partial_{x_{1}}\left(\left[\phi w^{1}\right]_{1}-\left[\phi^{(0)} w^{(0), 1} \sigma_{1}^{2}\right]_{1}\right) \\
+\partial_{x_{1}} \mathscr{P}^{(1)}(z)\left(\sigma_{1}^{2} \boldsymbol{F}_{1}(z)+\boldsymbol{F}_{2}(z)\right)+\partial_{x_{1}}^{2} \mathscr{P}^{(2)}(z) \boldsymbol{F}(z)
\end{gathered}
$$

Here $a_{11}(z)=\left[\phi^{(0)} w^{(0), 1}(z)\right]$. Since

$$
\mathscr{F}\left\{\mathscr{P}^{(1)}(z)\left(\sigma_{1}^{2} \boldsymbol{F}_{1}(z)\right)\right\}=\widehat{\chi}_{1}\left\langle\widehat{\left(\sigma_{1}^{2}\right)} \boldsymbol{F}_{1}(z), u^{*(1)}(z)\right\rangle=\widehat{\chi}_{1}\left\langle\boldsymbol{F}_{1}(z), u^{*(1)}(z)\right\rangle \widehat{\left(\sigma_{1}^{2}\right)}=-a_{12}(z) \sigma_{1}^{2},
$$

where $a_{12}(z)=-\left\langle\boldsymbol{F}_{1}(z), u^{*(1)}(z)\right\rangle$.

Using properties of $e^{(t-z) \Lambda}$, we thus arrive at

$$
\begin{gathered}
e^{(t-z) \Lambda} \mathscr{P}(z) \boldsymbol{F}(z)=-a_{1}(z) e^{(t-z) \Lambda} \partial_{x_{1}}\left(\sigma_{1}^{2}(z)\right) \\
-e^{(t-z) \Lambda} \partial_{x_{1}}\left\{\left[\phi w^{1}\right]_{1}(z)-\left[\phi^{(0)} w^{(0), 1}(z)\right] \sigma_{1}^{2}(z)\right\} \\
+e^{(t-z) \Lambda} h_{5}(z)+e^{(t-z) \Lambda} h_{6}(z),
\end{gathered}
$$

where $a_{1}(z)=a_{11}(z)+a_{12}(z), \sup _{z \in J_{T}}\left|a_{1}(z)\right| \leq C$ and

$$
\begin{aligned}
h_{5}(z)= & \partial_{x_{1}} \mathscr{P}^{(1)}(z) \boldsymbol{F}_{2}(z)+\partial_{x_{1}}^{2} \mathscr{P}^{(2)}(z) \boldsymbol{F}_{2}(z), \\
& h_{6}(z)=\partial_{x_{1}}^{2} \mathscr{P}^{(2)}(z)\left(\sigma_{1}^{2} \boldsymbol{F}_{1}(z)\right) .
\end{aligned}
$$

It then follows from (9.2) and (9.3) that

$$
\sigma_{1}(t)-\sigma(t)=\sum_{j=0}^{6} I_{j}(t)
$$

where 


$$
\begin{gathered}
I_{0}(t)=e^{t \Lambda} \mathscr{P}(0) u_{0}-\mathscr{H}(t) \sigma_{0}, \\
I_{1}(t)=-\int_{0}^{t} \omega_{0} \mathscr{H}(t-z) \partial_{x_{1}}\left(\sigma_{1}^{2}(z)-\sigma^{2}(z)\right) d z \\
I_{2}(t)=-\int_{0}^{t} \omega_{0}\left(e^{(t-z) \Lambda}-\mathscr{H}(t-z)\right) \partial_{x_{1}}\left(\sigma_{1}^{2}\right) d z, \\
I_{3}(t)=-\int_{0}^{t}\left(a_{1}(z)-\omega_{0}\right) e^{(t-z) \Lambda} \partial_{x_{1}}\left(\sigma_{1}^{2}\right) d z, \\
I_{4}(t)=-\int_{0}^{t} \partial_{x_{1}} e^{(t-z) \Lambda}\left(\left[\phi w^{1}\right]_{1}(z)-\left[\phi^{(0)} w^{(0), 1}(z)\right] \sigma_{1}^{2}(z)\right) d z, \\
I_{j}(t)=\int_{0}^{t} e^{(t-z) \Lambda} h_{j}(z) d z, \quad j=5,6 .
\end{gathered}
$$

Let us show estimates on $I_{j}, j=0, \ldots, 6$.

As for $I_{0}$, from (4.10) we see

$$
\left\|I_{0}(t)\right\|_{H^{1}} \leq C t^{-\frac{3}{4}}\left\|u_{0}\right\|_{L^{1}}
$$

Let us consider $I_{1}(t)$. By Lemma 9.3, (5.12) and the definition of $M(t)$ and $N(t)$, we have

$$
\left\|\left(\sigma_{1}^{2}-\sigma^{2}\right)(z)\right\|_{1} \leq\left\|\left(\sigma_{1}+\sigma\right)(z)\right\|_{2}\left\|\left(\sigma_{1}-\sigma\right)(z)\right\|_{2} \leq C(1+z)^{-1+\delta} N(t)\left\|u_{0}\right\|_{H^{m} \cap L^{1}},
$$

for $\left\|u_{0}\right\|_{H^{m} \cap L^{1}} \leq \varepsilon_{3}$. Furthermore, by Lemma 8.2 (iii) we have $\left\|\left(\sigma_{1}-\sigma\right)(z)\right\|_{\infty} \leq C(1+z)^{-\frac{3}{4}+\delta} N(t)$, and hence,

$$
\begin{gathered}
\left\|\partial_{x_{1}}\left(\sigma_{1}^{2}-\sigma^{2}\right)(z)\right\|_{2} \leq C\left\{\left\|\left(\sigma_{1}+\sigma\right)(z)\right\|_{\infty}\left\|\partial_{x_{1}}\left(\sigma_{1}-\sigma\right)(z)\right\|_{2}+\left\|\left(\sigma_{1}-\sigma\right)(z)\right\|_{\infty}\left\|\partial_{x_{1}}\left(\sigma_{1}+\sigma\right)(z)\right\|_{2}\right\} \\
\leq C(1+z)^{-\frac{5}{4}+\delta}\left\|u_{0}\right\|_{H^{m} \cap L^{1}} N(t) .
\end{gathered}
$$

It then follows from (4.8) that for $k=0,1$,

$$
\begin{gathered}
\left\|\partial_{x_{1}}^{k} I_{1}(t)\right\|_{2} \leq C\left\{\int_{0}^{\frac{t-1}{2}}(t-z)^{-\frac{3}{4}-\frac{k}{2}}(1+z)^{-1+\delta} d z+\int_{\frac{t-1}{2}}^{t-\frac{1}{2}}(t-z)^{-\frac{3}{4}-\frac{k}{2}}(1+z)^{-1+\delta} d z\right. \\
\left.+\int_{t-\frac{1}{2}}^{t}(t-z)^{-\frac{k}{2}}(1+z)^{-\frac{5}{4}+\delta} d z\right\}\left\|u_{0}\right\|_{H^{m} \cap L^{1}} N(t) \leq C(1+t)^{-\frac{3}{4}+\delta}\left\|u_{0}\right\|_{H^{m} \cap L^{1}} N(t) .
\end{gathered}
$$

As for $I_{2}(t)$, we see from (4.11) that for $k=0,1$,

$\left\|\partial_{x_{1}}^{k} I_{2}(t)\right\|_{2} \leq C\left\{\int_{0}^{\frac{t-1}{2}}(t-z)^{-\frac{5}{4}-\frac{k}{2}}\left\|\sigma_{1}^{2}(z)\right\|_{1} d z+\int_{\frac{t-1}{2}}^{t-\frac{1}{2}}(t-z)^{-\frac{3}{4}-\frac{k}{2}}\left\|\partial_{x_{1}}\left(\sigma_{1}^{2}(z)\right)\right\|_{1} d z+\int_{t-\frac{1}{2}}^{t}(t-z)^{-\frac{k}{2}}\left\|\partial_{x_{1}}\left(\sigma_{1}^{2}(z)\right)\right\|_{2} d z\right\}$.

From Lemma 6.3 we have

$$
\begin{gathered}
\leq C\left\{\int_{0}^{\frac{t-1}{2}}(t-z)^{-\frac{5}{4}-\frac{k}{2}}(1+z)^{-\frac{1}{2}} d z+\int_{\frac{t-1}{2}}^{t-\frac{1}{2}}(t-z)^{-\frac{3}{4}-\frac{k}{2}}(1+z)^{-1} d z+\int_{t-\frac{1}{2}}^{t}(t-z)^{-\frac{k}{2}}(1+z)^{-\frac{5}{4}} d z\right\} M(t)^{2} \\
\leq C(1+t)^{-\frac{3}{4}}\left\|u_{0}\right\|_{H^{m} \cap L^{1}}^{2} .
\end{gathered}
$$

As for $I_{3}(t)$, let us define $b(t)=\int_{0}^{t} a_{1}(z)-\omega_{0} d z$. Then $\partial_{t} b(t)=a_{1}(t)-\omega_{0}$ and $b(0)=b(T)=0$. Since $a_{1}(t+T)=a_{1}(t)$ we have $\partial_{t} b(t+T)=\partial_{t} b(t)$ and thus $b(t+T)=b(t)$. We arrive at $\sup _{z \in J_{T}}|b(z)| \leq C$. We write

$$
I_{3}(t)=-\int_{0}^{t} \partial_{z} b(z) e^{(t-z) \Lambda} \partial_{x_{1}}\left(\sigma_{1}^{2}\right) d z=-\left[b(z) e^{(t-z) \Lambda} \partial_{x_{1}}\left(\sigma_{1}^{2}(z)\right)\right]_{0}^{t}+\int_{0}^{t} b(z) \partial_{z}\left(e^{(t-z) \Lambda} \partial_{x_{1}}\left(\sigma_{1}^{2}(z)\right)\right) d z
$$


$=-b(t) \partial_{x_{1}}\left(\sigma_{1}^{2}(t)\right)-\int_{0}^{t} b(z) e^{(t-z) \Lambda} \Lambda \partial_{x_{1}}\left(\sigma_{1}^{2}(z)\right) d z+\int_{0}^{t} b(z) \partial_{x_{1}} e^{(t-z) \Lambda} \partial_{z}\left(\sigma_{1}^{2}(z)\right) d z \equiv J_{1}(t)+J_{2}(t)+J_{3}(t)$.

From Lemma 6.3 (vii) we have for $k=0,1$,

$$
\left\|\partial_{x_{1}}^{k} J_{1}(t)\right\|_{2} \leq C(1+t)^{-\frac{5}{4}} M(t)^{2} .
$$

We see from (4.9) and Lemma 6.3 that

$$
\begin{gathered}
\left\|J_{2}(t)\right\|_{2} \leq C\left\{\int_{0}^{\frac{t}{2}}(1+t-z)^{-\frac{5}{4}}\left\|\sigma_{1}^{2}(z)\right\|_{1} d z+\int_{\frac{t}{2}}^{t}(1+t-z)^{-\frac{3}{4}}\left\|\partial_{x_{1}}\left(\sigma_{1}^{2}(z)\right)\right\|_{1} d z\right\} \\
\leq C(1+t)^{-\frac{3}{4}}\left\|u_{0}\right\|_{H^{m} \cap L^{1}}^{2} .
\end{gathered}
$$

As for $J_{3}$, using (6.3) we calculate

$$
J_{3}(t)=2 \int_{0}^{t} b(z) \partial_{x_{1}} e^{(t-z) \Lambda} \sigma_{1}(z) \Lambda \sigma_{1}(z) d z+2 \int_{0}^{t} b(z) \partial_{x_{1}} e^{(t-z) \Lambda} \sigma_{1}(z) \mathscr{P}(z) \boldsymbol{F}(z) d z \equiv J_{31}+J_{32} .
$$

Using (4.9) and Lemma 6.3 we calculate

$$
\begin{aligned}
\left\|J_{31}\right\|_{2} & \leq C \int_{0}^{t}(1+t-z)^{-\frac{3}{4}}\left\|\sigma_{1}(z) \Lambda \sigma_{1}(z)\right\|_{1} d z \leq C \int_{0}^{t}(1+t-z)^{-\frac{3}{4}}\left\|\sigma_{1}(z)\right\|_{2}\left\|\Lambda \sigma_{1}(z)\right\|_{2} d z \\
& \leq C M(t)^{2} \int_{0}^{t}(1+t-z)^{-\frac{3}{4}}(1+z)^{-1} d z \leq C(1+t)^{-\frac{3}{4}} \log (1+t)\left\|u_{0}\right\|_{H^{m} \cap L^{1}}^{2} .
\end{aligned}
$$

Analogously we obtain for $J_{32}$ that

$$
\left\|J_{32}\right\|_{2} \leq C \int_{0}^{t}(1+t-z)^{-\frac{3}{4}}\left\|\sigma_{1}(z)\right\|_{2}\|\boldsymbol{F}(z)\|_{2} d z \leq C(1+t)^{-\frac{3}{4}} \log (1+t)\left\|u_{0}\right\|_{H^{m} \cap L^{1}}^{3} .
$$

As for $I_{4}(t)$, we have

$$
\begin{gathered}
\left\|\left[\phi w^{1}\right]_{1}(z)-\left[\phi^{(0)} w^{(0), 1}(z)\right] \sigma_{1}^{2}(z)\right\|_{1} \leq C\left\{\left\|\sigma_{1}(z)\right\|_{2}\left\|u(z)-\sigma_{1}(z) u^{(0)}(z)\right\|_{2}+\left\|u(z)-\sigma_{1}(z) u^{(0)}(z)\right\|_{2}^{2}\right\} \\
\leq C(1+z)^{-1} M(z)^{2} .
\end{gathered}
$$

Thus, (4.9) gives us

$$
\left\|\partial_{x_{1}}^{k} I_{4}(t)\right\|_{2} \leq C M(t)^{2} \int_{0}^{t}(1+t-z)^{-\frac{3}{4}-\frac{k}{2}}(1+z)^{-1} d z \leq C(1+t)^{-\frac{3}{4}} \log (1+t)\left\|u_{0}\right\|_{H^{m} \cap L^{1}}^{2} .
$$

To estimate $I_{5}(t)$, we write $h_{5}(z)$ as

$$
h_{5}(z)=\partial_{x_{1}}\left(\mathscr{P}^{(1)}(z) \boldsymbol{F}_{2}(z)+\partial_{x_{1}} \mathscr{P}^{(2)}(z) \boldsymbol{F}_{2}(z)\right) .
$$

Using (4.14) and Lemma $6.3(\mathrm{v})$, we have

$$
\left\|\partial_{x_{1}}^{k} I_{5}(t)\right\|_{2} \leq C M(t)^{2} \int_{0}^{t}(1+t-z)^{-\frac{3}{4}-\frac{k}{2}}(1+z)^{-1} d z \leq C(1+t)^{-\frac{3}{4}} \log (1+t)\left\|u_{0}\right\|_{H^{m} \cap L^{1}}^{2} .
$$

As for $I_{6}(t)$, we write $h_{6}(z)$ as

$$
h_{6}(z)= \begin{cases}\partial_{x_{1}}^{2} \mathscr{P}^{(2)}(z)\left(\sigma_{1}^{2} \boldsymbol{F}_{1}\right)(z) & \text { for } z \in\left[0, \frac{t}{2}\right], \\ \partial_{x_{1}} \mathscr{P}^{(2)}(z)\left(\partial_{x_{1}}\left(\sigma_{1}^{2}\right) \boldsymbol{F}_{1}\right)(z) & \text { for } z \in\left[\frac{t}{2}, t\right] .\end{cases}
$$

We see from (4.14) and Lemma 6.3 that

$$
\left\|\partial_{x_{1}}^{k} I_{6}(t)\right\|_{2} \leq C\left\{\int_{0}^{\frac{t}{2}}(1+t-z)^{-\frac{5}{4}-\frac{k}{2}}\left\|\sigma_{1}^{2}(z)\right\|_{1} d z+\int_{\frac{t}{2}}^{t}(1+t-z)^{-\frac{3}{4}-\frac{k}{2}}\left\|\partial_{x_{1}}\left(\sigma_{1}\right)^{2}\right\|_{1} d z\right\} M(t)^{2}
$$




$$
\leq C\left\{\int_{0}^{\frac{t}{2}}(1+t-z)^{-\frac{5}{4}-\frac{k}{2}}(1+z)^{-\frac{1}{2}} d z+\int_{\frac{t}{2}}^{t}(1+t-z)^{-\frac{3}{4}-\frac{k}{2}}(1+z)^{-1} d z\right\} M(t)^{2} \leq C(1+t)^{-\frac{3}{4}}\left\|u_{0}\right\|_{H^{m} \cap L^{1}}^{2} .
$$

We thus obtain

$$
\left\|\left(\sigma_{1}-\sigma\right)(t)\right\|_{H^{1}} \leq C(1+t)^{-\frac{3}{4}+\delta}\left\|u_{0}\right\|_{H^{m} \cap L^{1}}\left\{1+\left\|u_{0}\right\|_{H^{m} \cap L^{1}}+\left\|u_{0}\right\|_{H^{m} \cap L^{1}}^{2}+N(t)\right\},
$$

which yields

$$
N(t) \leq\left\|u_{0}\right\|_{H^{m} \cap L^{1}}\left\{1+\left\|u_{0}\right\|_{H^{m} \cap L^{1}}+\left\|u_{0}\right\|_{H^{m} \cap L^{1}}^{2}+N(t)\right\} .
$$

The desired result now follows by taking $\left\|u_{0}\right\|_{H^{m} \cap L^{1}}$ suitably small. This completes the proof.

Now let us show the asymptotic behavior in cases $n \geq 3$.

Theorem 9.4 Let $n \geq 3$. There exists $\varepsilon_{8}>0$ such that if $\left\|u_{0}\right\|_{H^{m} \cap L^{1}} \leq \varepsilon_{8}$, then

$$
\left\|\sigma_{1}(t)-\mathscr{H}(t) \sigma_{0}\right\|_{2} \leq C(1+t)^{-\frac{n+1}{4}} \eta_{n}(t)\left\|u_{0}\right\|_{H^{m} \cap L^{1}}
$$

where $\eta_{n}(t)=\log (1+t)$ when $n=3$ and $\eta_{n}(t)=1$ when $n \geq 4$ and $t \geq 0$.

Proof. From (9.3) we see that

$$
\sigma_{1}(t)-\mathscr{H}(t) \sigma_{0}=e^{t \Lambda} \mathscr{P}(0) u_{0}-\mathscr{H}(t) \sigma_{0}+\int_{0}^{t} e^{(t-z) \Lambda} \mathscr{P}(z) \boldsymbol{F}(z) d z .
$$

Estimate (4.10) then implies

$$
\left\|e^{t \Lambda} \mathscr{P}(0) u_{0}-\mathscr{H}(t) \sigma_{0}\right\|_{2} \leq C t^{-\frac{n-1}{4}-\frac{1}{2}}\left\|u_{0}\right\|_{L^{1}(\Omega)} .
$$

By Lemma 6.1 (ii) we have

$$
\mathscr{P}(z) \boldsymbol{F}(z)=-\operatorname{div}^{\prime}\left[\phi(z) w^{\prime}(z)\right]_{1}+\operatorname{div}^{\prime} \mathscr{P}^{(1)}(z) \boldsymbol{F}(z)+\Delta^{\prime} \mathscr{P}^{(2)}(z) \boldsymbol{F}(z),
$$

and thus by using (4.14) and Lemma 6.3 we obtain

$$
\begin{aligned}
& \left\|\int_{0}^{t} e^{(t-z) \Lambda} \mathscr{P}(z) \boldsymbol{F}(z) d z\right\|_{2} \leq C \int_{0}^{t}(1+t-z)^{-\frac{n-1}{4}-\frac{1}{2}}\left(\left\|\left[\phi w^{\prime}\right](z)\right\|_{1}+\|\boldsymbol{F}(z)\|_{1}\right) d z \\
\leq & C M(t)^{2} \int_{0}^{t}(1+t-z)^{-\frac{n-1}{4}-\frac{1}{2}}(1+z)^{-\frac{n-1}{2}} d z \leq C(1+t)^{-\frac{n-1}{4}-\frac{1}{2}} \eta_{n}(t)\left\|u_{0}\right\|_{H^{m} \cap L^{1}} .
\end{aligned}
$$

This concludes the proof.

(3.9) now follows from (5.13) and Theorem 9.4.

\section{References}

[1] Brezina, J., Kagei, Y. (2012). Decay properties of solutions to the linearized compressible Navier-Stokes equation around time-periodic parallel flow. Mathematical Models and Methods in Applied Sciences, Vol. 22, No. 7, pp.1250007-1-1250007-53.

[2] Brezina, J., Kagei, Y. (2012). Spectral properties of the linearized compressible Navier-Stokes equation around time-periodic parallel flow. MI Preprint Series 2012-9, Kyushu University.

[3] Galdi, G. P. (1994). An Introdction to the Mathematical Theory of the Navier-Stokes Equations. Vol. 1, Springer-Verlag New York

[4] Kagei, Y. (2008). Large time behavior of solutions to the compressible Navier-Stokes eqution in an infinite layer. Hiroshima Math. J. 38, pp.95-124.

[5] Kagei, Y. (2011). Asymptotic behavior of solutions of the compressible Navier-Stokes equation around the plane Couette flow. J. Math. Fluid Mech. Vol.13, pp.1-31. 
[6] Kagei, Y. (2011). Global existence of solutions to the compressible Navier-Stokes equation around parallel flows. J. Differential Equations. 251, pp.3248-3295.

[7] Kagei, Y. (2012). Asymptotic behavior of the solutions to the compressible Navier-Stokes equation around a parallel flow. Arch. Rational Mech. Anal. Vol. 205, pp.585-650.

[8] Kagei, Y., Kawashima, S. (2006). Local solvability of initial boundary value problem for a quasilinear hyperbolic-parabolic system. J. Hyperbolic Differential Equations, 3, pp.195-232.

[9] Kagei, Y., Kawashima, S. (2006). Stability of planar stationary solutions to the compressible NavierStokes equation on the half space. Comm. Math. Phys., 266, pp.401-430.

[10] Kagei, Y., Kobayashi, T. (2005). Asymptotic behavior of solutions to the compressible Navier-Stokes equations on the half space. Arch. Rational Mech. Anal., 177, pp.213-330.

[11] Kagei, Y., Nagafuchi, Y., Sudou, T. (2010). Decay estimates on solutions of the linearized compressible Navier-Stokes equation around a Poiseuille type flow. J. Math-for-Ind. 2A, pp.39-56. Correction to "Decay estimates on solutions of the linearized compressible Navier-Stokes equation around a Poiseuille type flow" in J. Math-for-Ind. 2A (2010), pp.39-56 J. Math-for-Ind. 2B (2010), pp.235.

[12] Matsumura, A., Nishida, T. (1983). Initial boundary value problems for the equations of motion of compressible viscous and heat-conductive fluids. Commun. Math. Phys. 89, pp.445-464. 


\section{List of MI Preprint Series, Kyushu University \\ The Global COE Program \\ Math-for-Industry Education \& Research Hub}

MI

MI2008-1 Takahiro ITO, Shuichi INOKUCHI \& Yoshihiro MIZOGUCHI

Abstract collision systems simulated by cellular automata

MI2008-2 Eiji ONODERA

The intial value problem for a third-order dispersive flow into compact almost Hermitian manifolds

MI2008-3 Hiroaki KIDO

On isosceles sets in the 4-dimensional Euclidean space

MI2008-4 Hirofumi NOTSU

Numerical computations of cavity flow problems by a pressure stabilized characteristiccurve finite element scheme

MI2008-5 Yoshiyasu OZEKI

Torsion points of abelian varieties with values in nfinite extensions over a p-adic field

MI2008-6 Yoshiyuki TOMIYAMA

Lifting Galois representations over arbitrary number fields

MI2008-7 Takehiro HIROTSU \& Setsuo TANIGUCHI

The random walk model revisited

MI2008-8 Silvia GANDY, Masaaki KANNO, Hirokazu ANAI \& Kazuhiro YOKOYAMA

Optimizing a particular real root of a polynomial by a special cylindrical algebraic decomposition

MI2008-9 Kazufumi KIMOTO, Sho MATSUMOTO \& Masato WAKAYAMA

Alpha-determinant cyclic modules and Jacobi polynomials

MI2008-10 Sangyeol LEE \& Hiroki MASUDA

Jarque-Bera Normality Test for the Driving Lévy Process of a Discretely Observed Univariate SDE

MI2008-11 Hiroyuki CHIHARA \& Eiji ONODERA

A third order dispersive flow for closed curves into almost Hermitian manifolds

MI2008-12 Takehiko KINOSHITA, Kouji HASHIMOTO and Mitsuhiro T. NAKAO

On the $L^{2}$ a priori error estimates to the finite element solution of elliptic problems with singular adjoint operator

MI2008-13 Jacques FARAUT and Masato WAKAYAMA

Hermitian symmetric spaces of tube type and multivariate Meixner-Pollaczek polynomials 
MI2008-14 Takashi NAKAMURA

Riemann zeta-values, Euler polynomials and the best constant of Sobolev inequality

MI2008-15 Takashi NAKAMURA

Some topics related to Hurwitz-Lerch zeta functions

MI2009-1 Yasuhide FUKUMOTO

Global time evolution of viscous vortex rings

MI2009-2 Hidetoshi MATSUI \& Sadanori KONISHI

Regularized functional regression modeling for functional response and predictors

MI2009-3 Hidetoshi MATSUI \& Sadanori KONISHI

Variable selection for functional regression model via the $L_{1}$ regularization

MI2009-4 Shuichi KAWANO \& Sadanori KONISHI

Nonlinear logistic discrimination via regularized Gaussian basis expansions

MI2009-5 Toshiro HIRANOUCHI \& Yuichiro TAGUCHII

Flat modules and Groebner bases over truncated discrete valuation rings

MI2009-6 Kenji KAJIWARA \& Yasuhiro OHTA

Bilinearization and Casorati determinant solutions to non-autonomous $1+1$ dimensional discrete soliton equations

MI2009-7 Yoshiyuki KAGEI

Asymptotic behavior of solutions of the compressible Navier-Stokes equation around the plane Couette flow

MI2009-8 Shohei TATEISHI, Hidetoshi MATSUI \& Sadanori KONISHI

Nonlinear regression modeling via the lasso-type regularization

MI2009-9 Takeshi TAKAISHI \& Masato KIMURA

Phase field model for mode III crack growth in two dimensional elasticity

MI2009-10 Shingo SAITO

Generalisation of Mack's formula for claims reserving with arbitrary exponents for the variance assumption

MI2009-11 Kenji KAJIWARA, Masanobu KANEKO, Atsushi NOBE \& Teruhisa TSUDA Ultradiscretization of a solvable two-dimensional chaotic map associated with the Hesse cubic curve

MI2009-12 Tetsu MASUDA

Hypergeometric T -functions of the q-Painlevé system of type $E_{8}^{(1)}$

MI2009-13 Hidenao IWANE, Hitoshi YANAMI, Hirokazu ANAI \& Kazuhiro YOKOYAMA A Practical Implementation of a Symbolic-Numeric Cylindrical Algebraic Decomposition for Quantifier Elimination

MI2009-14 Yasunori MAEKAWA

On Gaussian decay estimates of solutions to some linear elliptic equations and its applications 
MI2009-15 Yuya ISHIHARA \& Yoshiyuki KAGEI

Large time behavior of the semigroup on $L^{p}$ spaces associated with the linearized compressible Navier-Stokes equation in a cylindrical domain

MI2009-16 Chikashi ARITA, Atsuo KUNIBA, Kazumitsu SAKAI \& Tsuyoshi SAWABE

Spectrum in multi-species asymmetric simple exclusion process on a ring

MI2009-17 Masato WAKAYAMA \& Keitaro YAMAMOTO

Non-linear algebraic differential equations satisfied by certain family of elliptic functions

MI2009-18 Me Me NAING \& Yasuhide FUKUMOTO

Local Instability of an Elliptical Flow Subjected to a Coriolis Force

MI2009-19 Mitsunori KAYANO \& Sadanori KONISHI

Sparse functional principal component analysis via regularized basis expansions and its application

MI2009-20 Shuichi KAWANO \& Sadanori KONISHI

Semi-supervised logistic discrimination via regularized Gaussian basis expansions

MI2009-21 Hiroshi YOSHIDA, Yoshihiro MIWA \& Masanobu KANEKO

Elliptic curves and Fibonacci numbers arising from Lindenmayer system with symbolic computations

MI2009-22 Eiji ONODERA

A remark on the global existence of a third order dispersive flow into locally Hermitian symmetric spaces

MI2009-23 Stjepan LUGOMER \& Yasuhide FUKUMOTO

Generation of ribbons, helicoids and complex scherk surface in laser-matter Interactions

MI2009-24 Yu KAWAKAMI

Recent progress in value distribution of the hyperbolic Gauss map

MI2009-25 Takehiko KINOSHITA \& Mitsuhiro T. NAKAO

On very accurate enclosure of the optimal constant in the a priori error estimates for $H_{0}^{2}$-projection

MI2009-26 Manabu YOSHIDA

Ramification of local fields and Fontaine's property (Pm)

MI2009-27 Yu KAWAKAMI

Value distribution of the hyperbolic Gauss maps for flat fronts in hyperbolic threespace

MI2009-28 Masahisa TABATA

Numerical simulation of fluid movement in an hourglass by an energy-stable finite element scheme

MI2009-29 Yoshiyuki KAGEI \& Yasunori MAEKAWA

Asymptotic behaviors of solutions to evolution equations in the presence of translation and scaling invariance 
MI2009-30 Yoshiyuki KAGEI \& Yasunori MAEKAWA

On asymptotic behaviors of solutions to parabolic systems modelling chemotaxis

MI2009-31 Masato WAKAYAMA \& Yoshinori YAMASAKI

Hecke's zeros and higher depth determinants

MI2009-32 Olivier PIRONNEAU \& Masahisa TABATA

Stability and convergence of a Galerkin-characteristics finite element scheme of lumped mass type

MI2009-33 Chikashi ARITA

Queueing process with excluded-volume effect

MI2009-34 Kenji KAJIWARA, Nobutaka NAKAZONO \& Teruhisa TSUDA

Projective reduction of the discrete Painlevé system of type $\left(A_{2}+A_{1}\right)^{(1)}$

MI2009-35 Yosuke MIZUYAMA, Takamasa SHINDE, Masahisa TABATA \& Daisuke TAGAMI Finite element computation for scattering problems of micro-hologram using DtN map

MI2009-36 Reiichiro KAWAI \& Hiroki MASUDA

Exact simulation of finite variation tempered stable Ornstein-Uhlenbeck processes

MI2009-37 Hiroki MASUDA

On statistical aspects in calibrating a geometric skewed stable asset price model

MI2010-1 Hiroki MASUDA

Approximate self-weighted LAD estimation of discretely observed ergodic OrnsteinUhlenbeck processes

MI2010-2 Reiichiro KAWAI \& Hiroki MASUDA

Infinite variation tempered stable Ornstein-Uhlenbeck processes with discrete observations

MI2010-3 Kei HIROSE, Shuichi KAWANO, Daisuke MIIKE \& Sadanori KONISHI

Hyper-parameter selection in Bayesian structural equation models

MI2010-4 Nobuyuki IKEDA \& Setsuo TANIGUCHI

The Itô-Nisio theorem, quadratic Wiener functionals, and 1-solitons

MI2010-5 Shohei TATEISHI \& Sadanori KONISHI

Nonlinear regression modeling and detecting change point via the relevance vector machine

MI2010-6 Shuichi KAWANO, Toshihiro MISUMI \& Sadanori KONISHI

Semi-supervised logistic discrimination via graph-based regularization

MI2010-7 Teruhisa TSUDA

UC hierarchy and monodromy preserving deformation

MI2010-8 Takahiro ITO

Abstract collision systems on groups 
MI2010-9 Hiroshi YOSHIDA, Kinji KIMURA, Naoki YOSHIDA, Junko TANAKA \& Yoshihiro MIWA

An algebraic approach to underdetermined experiments

MI2010-10 Kei HIROSE \& Sadanori KONISHI

Variable selection via the grouped weighted lasso for factor analysis models

MI2010-11 Katsusuke NABESHIMA \& Hiroshi YOSHIDA

Derivation of specific conditions with Comprehensive Groebner Systems

MI2010-12 Yoshiyuki KAGEI, Yu NAGAFUCHI \& Takeshi SUDOU

Decay estimates on solutions of the linearized compressible Navier-Stokes equation around a Poiseuille type flow

MI2010-13 Reiichiro KAWAI \& Hiroki MASUDA

On simulation of tempered stable random variates

MI2010-14 Yoshiyasu OZEKI

Non-existence of certain Galois representations with a uniform tame inertia weight

MI2010-15 Me Me NAING \& Yasuhide FUKUMOTO

Local Instability of a Rotating Flow Driven by Precession of Arbitrary Frequency

MI2010-16 Yu KAWAKAMI \& Daisuke NAKAJO

The value distribution of the Gauss map of improper affine spheres

MI2010-17 Kazunori YASUTAKE

On the classification of rank 2 almost Fano bundles on projective space

MI2010-18 Toshimitsu TAKAESU

Scaling limits for the system of semi-relativistic particles coupled to a scalar bose field

MI2010-19 Reiichiro KAWAI \& Hiroki MASUDA

Local asymptotic normality for normal inverse Gaussian Lévy processes with highfrequency sampling

MI2010-20 Yasuhide FUKUMOTO, Makoto HIROTA \& Youichi MIE

Lagrangian approach to weakly nonlinear stability of an elliptical flow

MI2010-21 Hiroki MASUDA

Approximate quadratic estimating function for discretely observed Lévy driven SDEs with application to a noise normality test

MI2010-22 Toshimitsu TAKAESU

A Generalized Scaling Limit and its Application to the Semi-Relativistic Particles System Coupled to a Bose Field with Removing Ultraviolet Cutoffs

MI2010-23 Takahiro ITO, Mitsuhiko FUJIO, Shuichi INOKUCHI \& Yoshihiro MIZOGUCHI Composition, union and division of cellular automata on groups

MI2010-24 Toshimitsu TAKAESU

A Hardy's Uncertainty Principle Lemma in Weak Commutation Relations of HeisenbergLie Algebra 
MI2010-25 Toshimitsu TAKAESU

On the Essential Self-Adjointness of Anti-Commutative Operators

MI2010-26 Reiichiro KAWAI \& Hiroki MASUDA

On the local asymptotic behavior of the likelihood function for Meixner Lévy processes under high-frequency sampling

MI2010-27 Chikashi ARITA \& Daichi YANAGISAWA

Exclusive Queueing Process with Discrete Time

MI2010-28 Jun-ichi INOGUCHI, Kenji KAJIWARA, Nozomu MATSUURA \& Yasuhiro OHTA Motion and Bäcklund transformations of discrete plane curves

MI2010-29 Takanori YASUDA, Masaya YASUDA, Takeshi SHIMOYAMA \& Jun KOGURE On the Number of the Pairing-friendly Curves

MI2010-30 Chikashi ARITA \& Kohei MOTEGI

Spin-spin correlation functions of the $q$-VBS state of an integer spin model

MI2010-31 Shohei TATEISHI \& Sadanori KONISHI

Nonlinear regression modeling and spike detection via Gaussian basis expansions

MI2010-32 Nobutaka NAKAZONO

Hypergeometric $\tau$ functions of the $q$-Painlevé systems of type $\left(A_{2}+A_{1}\right)^{(1)}$

MI2010-33 Yoshiyuki KAGEI

Global existence of solutions to the compressible Navier-Stokes equation around parallel flows

MI2010-34 Nobushige KUROKAWA, Masato WAKAYAMA \& Yoshinori YAMASAKI

Milnor-Selberg zeta functions and zeta regularizations

MI2010-35 Kissani PERERA \& Yoshihiro MIZOGUCHI

Laplacian energy of directed graphs and minimizing maximum outdegree algorithms

MI2010-36 Takanori YASUDA

CAP representations of inner forms of $S p(4)$ with respect to Klingen parabolic subgroup

MI2010-37 Chikashi ARITA \& Andreas SCHADSCHNEIDER

Dynamical analysis of the exclusive queueing process

MI2011-1 Yasuhide FUKUMOTO\& Alexander B. SAMOKHIN

Singular electromagnetic modes in an anisotropic medium

MI2011-2 Hiroki KONDO, Shingo SAITO \& Setsuo TANIGUCHI

Asymptotic tail dependence of the normal copula

MI2011-3 Takehiro HIROTSU, Hiroki KONDO, Shingo SAITO, Takuya SATO, Tatsushi TANAKA \& Setsuo TANIGUCHI

Anderson-Darling test and the Malliavin calculus

MI2011-4 Hiroshi INOUE, Shohei TATEISHI \& Sadanori KONISHI

Nonlinear regression modeling via Compressed Sensing 
MI2011-5 Hiroshi INOUE

Implications in Compressed Sensing and the Restricted Isometry Property

MI2011-6 Daeju KIM \& Sadanori KONISHI

Predictive information criterion for nonlinear regression model based on basis expansion methods

MI2011-7 Shohei TATEISHI, Chiaki KINJYO \& Sadanori KONISHI

Group variable selection via relevance vector machine

MI2011-8 Jan BREZINA \& Yoshiyuki KAGEI

Decay properties of solutions to the linearized compressible Navier-Stokes equation around time-periodic parallel flow

Group variable selection via relevance vector machine

MI2011-9 Chikashi ARITA, Arvind AYYER, Kirone MALLICK \& Sylvain PROLHAC

Recursive structures in the multispecies TASEP

MI2011-10 Kazunori YASUTAKE

On projective space bundle with nef normalized tautological line bundle

MI2011-11 Hisashi ANDO, Mike HAY, Kenji KAJIWARA \& Tetsu MASUDA

An explicit formula for the discrete power function associated with circle patterns of Schramm type

MI2011-12 Yoshiyuki KAGEI

Asymptotic behavior of solutions to the compressible Navier-Stokes equation around a parallel flow

MI2011-13 Vladimír CHALUPECKÝ \& Adrian MUNTEAN

Semi-discrete finite difference multiscale scheme for a concrete corrosion model: approximation estimates and convergence

MI2011-14 Jun-ichi INOGUCHI, Kenji KAJIWARA, Nozomu MATSUURA \& Yasuhiro OHTA Explicit solutions to the semi-discrete modified KdV equation and motion of discrete plane curves

MI2011-15 Hiroshi INOUE

A generalization of restricted isometry property and applications to compressed sensing

MI2011-16 Yu KAWAKAMI

A ramification theorem for the ratio of canonical forms of flat surfaces in hyperbolic three-space

MI2011-17 Naoyuki KAMIYAMA

Matroid intersection with priority constraints

MI2012-1 Kazufumi KIMOTO \& Masato WAKAYAMA

Spectrum of non-commutative harmonic oscillators and residual modular forms

MI2012-2 Hiroki MASUDA

Mighty convergence of the Gaussian quasi-likelihood random fields for ergodic Levy driven SDE observed at high frequency 
MI2012-3 Hiroshi INOUE

A Weak RIP of theory of compressed sensing and LASSO

MI2012-4 Yasuhide FUKUMOTO \& Youich MIE

Hamiltonian bifurcation theory for a rotating flow subject to elliptic straining field

MI2012-5 Yu KAWAKAMI

On the maximal number of exceptional values of Gauss maps for various classes of surfaces

MI2012-6 Marcio GAMEIRO, Yasuaki HIRAOKA, Shunsuke IZUMI, Miroslav KRAMAR, Konstantin MISCHAIKOW \& Vidit NANDA

Topological Measurement of Protein Compressibility via Persistence Diagrams

MI2012-7 Nobutaka NAKAZONO \& Seiji NISHIOKA

Solutions to a $q$-analog of Painlevé III equation of type $D_{7}^{(1)}$

MI2012-8 Naoyuki KAMIYAMA

A new approach to the Pareto stable matching problem

MI2012-9 Jan BREZINA \& Yoshiyuki KAGEI

Spectral properties of the linearized compressible Navier-Stokes equation around time-periodic parallel flow

MI2012-10 Jan BREZINA

Asymptotic behavior of solutions to the compressible Navier-Stokes equation around a time-periodic parallel flow 